\title{
PENSCRIPTIVE DEPTH-CONTROLLED ROBOTIC LASER OSTEOTOMY
}

\author{
by
}

Jamil Jivraj

BEng, Electrical Engineering, Ryerson University, 2010

MASc, Electrical \& Computer Engineering, Ryerson University, 2013

\author{
A dissertation \\ presented to Ryerson University \\ in partial fulfillment of the \\ requirements for the degree of \\ Doctor of Philosophy \\ in the program of
}

Electrical \& Computer Engineering

Toronto, Ontario, Canada, 2019

CJamil Jivraj, 2019 


\section{AUTHOR'S DECLARATION FOR ELECTRONIC SUBMISSION OF A DISSERTATION}

I hereby declare that I am the sole author of this dissertation. This is a true copy of the dissertation, including any required final revisions, as accepted by my examiners.

I authorize Ryerson University to lend this dissertation to other institutions or individuals for the purpose of scholarly research.

I further authorize Ryerson University to reproduce this dissertation by photocopying or by other means, in total or in part, at the request of other institutions or individuals for the purpose of scholarly research.

I understand that my dissertation may be made electronically available to the public. 
Penscriptive depth controlled robotic laser osteotomy

Doctor of Philosophy 2019

Jamil Jivraj

Electrical \& Computer Engineering

Ryerson University

\begin{abstract}
Bone cutting in surgery is currently done using un-intelligent tools that depend on the proficiency of the surgeon to prevent damage to underlying critical structures. As one can imagine, damage isn't always prevented. Iatrogenic damage to dura and sub-dural neural structures during osteotomical procedures such as a craniotomy can result in increased patient morbidity.

This dissertation proposes the development of a robot-guided laser osteotome (bone cutter) with the use of inline optical coherence tomography (OCT) to precisely control the cutting depth in real-time. The all-fiber system design integrates a high peak-power pulsed Yb-doped fiber laser $(1064 \mathrm{~nm})$ coupled directly into the sample arm of a swept-source OCT system $\left(\lambda_{c}=1310 \mathrm{~nm}\right)$ with a fourth-order power disparity between the OCT system and fiber laser. Sub-millimeter accuracy was achieved in percussion drilling of phantom and porcine bone.

Through the use of optical topographic imaging (OTI), this work presents a novel method for the surgeon to identify arbitrary trajectories for desired cuts. A surgical pencil is used to demarcate cutting trajectories for the robot to follow directly onto the boney surface. OTI imaging combined with a novel algorithm developed through this work allows the penscribed line to be isolated and translated into spatial attitude information for the robot to guide the end effector-mounted laser along. Sub-millimeter trajectory following accuracy was achieved. This work also demonstrates the first use of OCT in continuous, real-time refocusing of the optical end-effector in order to main-
\end{abstract}


tain cut quality. The focus of the laser was able to be maintained within the Rayleigh length of the focused Gaussian beam for linear feed rates up to $1 \mathrm{~mm} / \mathrm{s}$ at a $45^{\circ}$ surface incline. Finally, optimization of bone ablation is explored in this dissertation. The use of graphite as a high-absorption topical chromophore and the use of nitrogen as an assist gas in the form of a coaxial jet are both analyzed to determine how to achieve the highest etch rate in bone. The results in this dissertation show that the topical application of graphite was able to significantly reduce the mean and variance of etching performance; an improvement by at least two orders of magnitude in the time to $0.5 \mathrm{~mm}$ etch depth is demonstrated. It is also demonstrated that etch rate during ablation can be optimized for coaxial nitrogen flow (30SCFH out of a nozzle with 3mm output diameter); higher and lower flow rates showed slower etch rates.

It is hypothesized that a system such as the one developed in this dissertation will increase the precision of bone cutting, decrease the amount of time needed to make cuts into sensitive structures and also address certain issues of unsuccessful uptake of lasers in modern medicine. 


\section{Acknowledgments}

Approaching the end of the $\mathrm{PhD}$ process demands reflection on the journey in it's entirety. During this contemplation, one comes to realize that this journey was not made in isolation, rather, that support has come from multiple hands and hearts. My name on the front page of this dissertation exists not only as a self-identifier, but also as a symbol representing everyone who has imparted intellectual and emotional momentum to my mass as it has travelled along this scholastic trajectory.

I am indebted to Dr. Victor Yang for supporting and guiding me throughout my $\mathrm{PhD}$. Taking his biophotonics graduate course during my master's was really the starting-point of how I got here. The intellectual exposure that Dr. Yang has given me, whether it's watching him perform surgery in the OR or having research conversations with him at his dinner table, has very much changed the way I practice engineering, scientific analysis and research.

Thank you to Dr. Xijia Gu for being my co-supervisor and for the many lessons, formal and informal, he's given me in lasers and optics. He's never turned me away anytime I've knocked on his door for help or advice. His expertise in laser physics and devices have been truly crucial for many parts of this dissertation.

Many thanks to the professors on my examination committee (Drs. Naimul Khan, Dimitri Androutsos, Krishnan Venkatakrishnan, Xaoyi Bao, Kristiina Mai and Paul Walsh).

I am very honoured to have worked with Chaoliang Chen, a post-doc in the BBL, but more-so a dear friend. Chao has been extremely beneficial to me in my development as a biophotonics researcher. One of the best decisions Dr. Yang had made was to have Chao and I share an office. This enabled me easy access to scientific discussions and advice on the construction and execution of experimental plans. Chao has been one of the best lab partners I've had throughout my time at Ryerson.

I am also grateful to Joel Ramjist, a great friend and the behind-the-scenes backbone of the lab. 
Even up till the night before the final defense, Joel has been a true help in my progression through this $\mathrm{PhD}$. Always ready to help and ever willing to self-sacrifice, Joel's compatriotism shall be forever valued.

As for my fellow BBL lab mates (Ryan Deorajh, Amy Weisong, Philips Lai, Nhu Ngyuen, Jillian Cardinelle, Nicole Dinn, Dorian), thanks for all the support and sharing some great laughs together. To all the BBL alumni (Tim Luk, Barry Vuong, Adrian miriampillai, Peter Seigler, Beau Standish, Kenneth Lee, Dexter Barrows), thanks for laying much of the technical groundwork that allowed my research to happen. Thank you Ronnie for starting the $\mathrm{PhD}$ journey with me; your comradery and contribution was vital to the naissance of this work. A big thank you to Eric $\mathrm{Lu}$ and Jiaqi (Terry) Zhou, Dr. Gu's former students, for teaching me how to build and repair (and repair, and repair, and repair...) the Yb-doped fiber laser used in this research. Also thank you Jenny Yeow for all the admin help you've provided throughout my time at the BBL. A side note, rest in peace Peter Morcos, a BBL lab mate, budding researcher, and personal friend whom we lost very suddenly and much too early. We all miss you, dude.

My friends have supported me in various ways through this degree and I'd like to mention some of these individuals by name. Thank you to Simon (Butters) for checking in on me with a call or a coffee to make sure I was doing okay. Thank you to Zahra, Shireen and Nis for rooting for me the whole way. Thanks Samantha and Josie for hang-outs and fun times. Thank you Yasmin for always lending an empathetic ear to my research woes. Thank you to Rizwan Rajabali, Hussein Kermally, Amira Jiwani, and all the RIMSA folks who were there to have a cup of chai and a laugh with. Thank you to Dr. Shafique Virani for being my informal sage, starting right from the application process and continuing all the way up to my first accepted publication and subsequent defenses; your wisdom is truly legion.

Finally, none of my academic work, from undergraduate till now, would have been possible without my family behind me. This is no over-statement; support from my family has been vital for me to complete my PhD. Alim, Tanaz, Kamran and Kian, thanks for having me over for many 
dinners and just to hang out at your place. These times were very welcome stress relievers (despite Kamran and Kian's loudness!) and a much needed break from staring at optical fibers and porcine scapulas all day. And to my parents, this dissertation is really a culmination of the sacrifices both of you have made to make sure I was able to pursue what I wanted to do. For this, I am the most indebted to both of you. The only way I can repay you is to keep trying to pursue some form of success, and not to forget that you both are present in whichever path I travel along next. I'm whole-heartedly grateful.

A quick thank you to Mac's/Circle K, Tim Horton's, 711, and Ideal Coffee (Kensington) for selling me all the coffee that the pages of this dissertation are dripping with. 


\section{Contents}

$\begin{array}{ll}\text { Abstract } & \text { ii }\end{array}$

List of Figures $\quad$ x

List of Tables $\quad$ Xv

$\begin{array}{ll}\text { List of Abbreviations } & \text { xvi }\end{array}$

1 Introduction $\quad 1$

1.1 Outline ............................. 5

2 Background 6

2.1 Osteotomy in spine and cranial surgery . . . . . . . . . . . . . . . 8

2.2 Laser osteotomy: techniques and challenges in modern medical uptake . . . . . . . 10

2.3 Laser interaction with osseous tissue . . . . . . . . . . . . . . . 11

2.4 Use of assist gas in laser material processing . . . . . . . . . . . . . . 16

2.5 OCT fundamentals . . . . . . . . . . . . . . . . . . . 17

2.5 .1 Interference of light . . . . . . . . . . . . . . . . . . . . . . . .

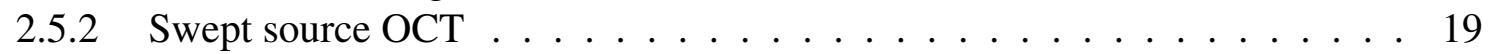

2.6 A brief history of neurosurgical robots . . . . . . . . . . . . . . 21

2.6.1 FDA approved neurosurgical robots . . . . . . . . . . . . . . 24

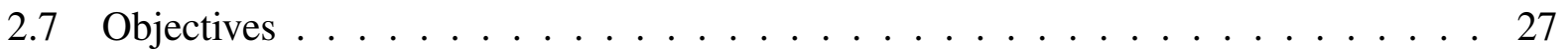

3 Fiber Laser and Inline OCT Integration 29

3.1 Introduction . . . . . . . . . . . . . . . . . . . . . . 29

3.1.1 OCT coupled ablation for process visualization . . . . . . . . . 30

3.2 Methods . . . . . . . . . . . . . . . . . . . 32

3.2 .1 Ablation fiber laser . . . . . . . . . . . . . . . 32

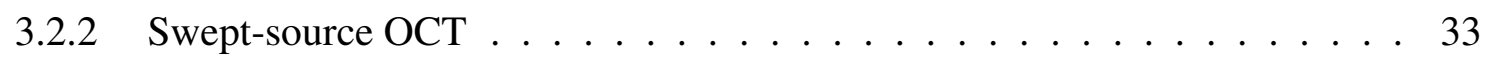

3.2.3 Fiber laser and OCT system coupling . . . . . . . . . . . . 33

3.2.4 A-line triggered ablation pulse phase delay . . . . . . . . . . . . . 37

3.2.5 Ablation depth control implementation . . . . . . . . . . . . . . 39

3.3 Experiments and Results . . . . . . . . . . . . . . . . . . . . . 42 
3.4 Discussion and Conclusion . . . . . . . . . . . . . . . . . . . . . 44

3.5 ADDENDUM 1: Alternate method for sample arm coupling of fiber laser . . . . . 46

3.6 ADDENDUM 2: Alternate method for k-clock delay . . . . . . . . . . . . . 49

4 Robotic Trajectory Generation via Structured Light Penscription 51

4.1 Introduction . . . . . . . . . . . . . . . . . . . . . . 51

4.1 .1 Related Work . . . . . . . . . . . . . . . . . . . . . . . 52

4.2 System Description . . . . . . . . . . . . . . . . . . . 53

4.2 .1 Vision system . . . . . . . . . . . . . . . . . . . . . 53

4.2 .2 Robotic hardware . . . . . . . . . . . . . . . . . . . 53

4.2 .3 System integration . . . . . . . . . . . . . . . . . 54

4.2 .4 Workflow . . . . . . . . . . . . . . . . . . 56

4.3 Algorithm . . . . . . . . . . . . . . . . . . . . . . . . 57

4.3 .1 Acquisition and Isolation . . . . . . . . . . . . . . . 57

4.3.2 Image space mapping and detection . . . . . . . . . . . . . . 57

4.3.3 Natural Direction Ordering and polynomial fit . . . . . . . . . . . . . 62

4.3 .4 Surface normal generation . . . . . . . . . . . . . . . . . 64

4.4 Experiments . . . . . . . . . . . . . . . . . . . 65

4.4.1 Deviation in target plane . . . . . . . . . . . . . . 65

4.4.2 Deviation along optical axis . . . . . . . . . . . . . . 66

4.4.3 Reproduction of surgical osteotomy trajectory . . . . . . . . . . . . 68

4.5 Discussion and Conclusion . . . . . . . . . . . . . . . . . . . . . 68

5 Bone Ablation Etch Rate Optimization Using Nitrogen as an Assist Gas and Graphite as a Topical Absorber $\quad 71$

5.1 Introduction . . . . . . . . . . . . . . . . . . . 71

5.1 .1 Topical chromophores . . . . . . . . . . . . . . . . . . . . 72

5.1.2 Graphite as a topical chromophore . . . . . . . . . . . . . . . . . 74

5.1 .3 Coaxial assist gas . . . . . . . . . . . . . . . . . . . . . . 75

5.2 Methods . . . . . . . . . . . . . . . . . . . . . 76

5.2.1 Ablation laser and in-line Optical Coherence Tomography system . . . . 76

5.3 Experimental Results . . . . . . . . . . . . . . . . . . . . . . 78

5.3.1 Reduction of ignition-time variance using a topical chromophore . . . . . 78

5.3 .2 Gas flow experiments . . . . . . . . . . . . . . . . . . . . . 79

5.3.3 Cortical bone experiments . . . . . . . . . . . . . . . . . 79

5.4 Discussion and Conclusion . . . . . . . . . . . . . . . . . . . . 81

6 Dynamic Axial Correction of Payload using OCT 84

6.1 Introduction . . . . . . . . . . . . . . . . . . . . . . . 84

6.2 System description $\ldots \ldots \ldots \ldots$. . . . . . . . . . . . . . . 86

6.2 .1 Hardware . . . . . . . . . . . . . . . . . . . . . . . 86

6.3 Experiments and Results . . . . . . . . . . . . . . . . . . . . . . . . 91

6.3 .1 Step response . . . . . . . . . . . . . . . . . . . . . 91 
6.3.2 Axial correction on sloped progression . . . . . . . . . . . . 91

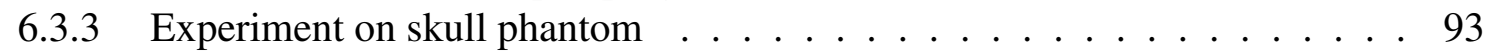

6.4 Discussion and Conclusions ........................... 95

7 Summary, Conclusions and Future Work 96

7.1 Future Work . . . . . . . . . . . . . . . . . . . . . 97

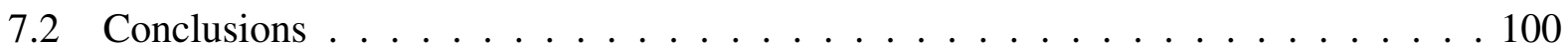

$\begin{array}{ll}\text { A Permissions } & 101\end{array}$

A.1 The Optical Society of America (Biomedical Optics Express) . . . . . . . . . 101

A.2 SPIE (Optical Engineering) . . . . . . . . . . . . . . . . 103

A.3 Springer Nature (International Journal of Computer Assisted Radiology and Surgery) 105 


\section{List of Figures}

2.1 Layers covering the brain $[1] \ldots \ldots \ldots \ldots \ldots \ldots$

2.2 Fundamental units of the two types of bone (cortical [2] and trabecular [3]). . . . 7

2.3 Details of the spinal column [4] and vertebrae [5] . . . . . . . . . . . . . 8

2.4 Common examples of cranial osteotomies. . . . . . . . . . . . . . . . . . . . 9

2.5 Absorption spectrum of water [6] . . . . . . . . . . . . . . . . . . . 14

2.6 Heat affected zone (HAZ) during laser tissue ablation $[7,8] \ldots \ldots$

2.7 A Michelson interferometer. . . . . . . . . . . . . . . . . . . . . . . . 18

2.8 Functional diagram of a swept source OCT system. . . . . . . . . . . . . 19

3.1 Schematic of the integrated OCT and fiber laser system. The entire fiber laser is built directly into the sample arm of the OCT system. Both systems are entirely fiber-based (aside from standard reference arm and focusing optics), allowing compact, robust packaging and elimination of dual-beam free-space alignment.(Reprinted with permission of the Optical Society of America) . . . . . . . . 34

3.2 OCT swept-source light ejection from fiber core due to bending. For the data shown in this figure bends of 10 different diameters were performed, repeated three times and averaged. The red line represents the Nufern SM-GDF-10/125, and the blue line represents standard SMF-28 fiber. It is important to note how dramatic the ejection from the core of the Nufern SM-GDF-10/125 fiber is, up to approximately $53 \mathrm{~mm}$ of bending diameter, compared to SMF-28. (Reprinted with permission of the Optical Society of America) . . . . . . . . . . . . . . . 35

3.3 Point spread function (PSF) of system with no K-clock timing consideration. Optical path difference-dependant peak broadening is very evident due to un-delayed sampling trigger. (Reprinted with permission of the Optical Society of America) . . 36

3.4 Point spread function (PSF) of the system with delayed MZI interferometer resampling clock to match round trip time of OCT light. Peak broadening is now approximately uniform due to high dispersion mismatch introduced by the inline fiber laser. (Reprinted with permission of the Optical Society of America) . . . . . 37

3.5 Point spread function (PSF) of the system with delayed MZI interferometer resampling clock to match round trip time of OCT light and $2^{\text {nd }}$ order software dispersion compensation. All axial resolution measurements are full-width, half-Max (FWHM). (Reprinted with permission of the Optical Society of America) . . . . . 38 
3.6 Timing relation between OCT data acquisition (A-line rate $=50 \mathrm{kHz}$ ) and ablation laser pulses $(50 \mathrm{kHz})$ when no synchronization is implemented (i.e. free-running ablation laser pulsing). The red box highlights which the portion of the cycle where the A-line acquisition takes place. It can be seen from the figure that the ablation pulses (c) clearly impinge on the OCT A-line (after the A-line trigger (a) and during the positive duty-cycle of the swept-source output (b). (Reprinted with permission of the Optical Society of America) . . . . . . . . . . . . . .

3.7 Depth progression of ablation front during ablation of wood (M-mode). Ablation laser induced artifacts are due to reflected energy through the fiber combiner saturating the balanced detectors. (Reprinted with permission of the Optical Society of America) . . . . . . . . . . . . . . . . . . . . . .

3.8 Timing relation between OCT data acquisition and ablation laser pulses with Aline triggered asymmetric pulse triggering timing scheme implemented. Red and blue boxes highlight portions of the cycle where A-line acquisition occurs and does not occur, respectively. Notice ablation (d) pulses occur only during the latter half of the cycle whilst no A-line acquisition is occuring (i.e. when swept-source output (b) is low). This is due to the fixed timing implemented between laser trigger pulse (c) and the A-line trigger (a). (Reprinted with permission of the Optical Society of

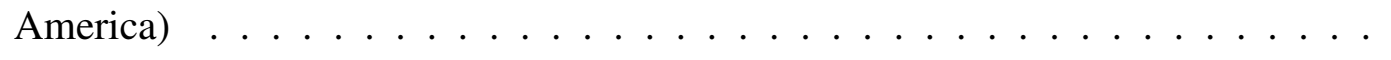

3.9 Depth progression of ablation front during ablation of wood (M-mode) using asymmetric pulse timing scheme. Ablation laser induced artifacts are no longer seen. (Reprinted with permission of the Optical Society of America) . . . . . . . .

3.10 Depth progression of ablation front during ablative irradiation of fresh porcine scapula (M-mode) using the asymmetric pulse timing scheme described above. A: heating of distal cortical bone surface; B: penetration through cortical layer and center trabecular layer; C: heating of proximal cortical bone layer; D: hole side wall reflection; E: laser shut off, subsequent tissue relaxation/contraction. (Reprinted with permission of the Optical Society of America) . . . . . . . . .

3.11 Diagram of system with integrated depth control. Detection of A-line peak at a target depth triggers a drive-signal shutoff to the ablation laser's pump diode driver. (Reprinted with permission of the Optical Society of America) . . . . . . . . . .

3.12 Ablation depth control trials on MDF phantom. Images a) to e) show OCT scans of the trials starting from $0.5 \mathrm{~mm}$ target depth to $2.5 \mathrm{~mm}$, incrementing by $0.5 \mathrm{~mm}$ respectively. Scale bars represent $0.5 \mathrm{~mm}$. Results are summarized in f). Red squares represent mean measured depths with standard deviation of each panel figure, indicated by the each corresponding label. (Reprinted with permission of the Optical Society of America) _ . . . . . . . . . . . . . . .

3.13 Schematic showing alternate method for coupling fiber laser into the sample arm of the OCT system. Note that this method eliminates the all-fiber design of the sample arm optics. . . . . . . . . . . . . . . . . 
3.14 Depth progression of ablation front during ablative irradiation of fresh lamb scapula (M-mode) using alternate coupling structure and software dispersion compensation. 1: ablation laser off; 2: ablation laser switched on; 3: ablation of superior cortical bone layer; 4: ablation of inner trabecular bone layer; 5 ablation of inferior cortical bone layer; 6: breach of inferior bone-air interface; 7: laser switched off ; *: side walls of ablated hole. . . . . . . . . . . . . . . . . 48

3.15 Schematic showing electrical based scheme for k-clock sample trigger delay. . . . 49

3.16 a) Point spread function (PSF) of the system with k-clock delay to match round trip time of OCT light. Peak broadening is now approximately uniform; irregular peak shapes are due to high dispersion introduced by the inline fiber laser. b) Point spread function (PSF) of the system with k-clock delay to match round trip time of OCT light and $2^{\text {nd }}$ order software dispersion compensation. Both figures show rising amplitude as a function of optical path difference, which is not to be expected. 50

4.1 Diagram of complete system. Tracking and structured light optics are housed in the surgical light head. Payload of robot guides the objective lens of the fiber laser ablation system over the target. Cartesian data is sent to the robot via ethernet by the navigation computer. . . . . . . . . . . . . .

4.2 Steps of the penscription detection algorithm. a) Phantom with penscription. b) View of the entire SL surface. c) Points picked by user (start point and approximate center). d) Mapping to flattened image space. e) Image dilation of mapped image. f) Gaussian blur of mapped image. g) Sobel edge detection filter. h) Nearest neighbour growth algorithm to detect residual points that represent penscription. i) Mapping back to image space. j) Skeletonization of trajectory via averaging. k) Trajectory after multi-order least squares polynomial fit. 1) Normals for endeffector orientation generated using principal component analysis. . . . . . . . . . 58

4.3 Error between penscription and ablated kerf (centerline-to-centerline) for a single straight line cutting trial. . . . . . . . . . . . . . . . . 66

4.4 Placement of target center in YZ-plane of the NDI Polaris tracking volume. The two red points represent the trials with the highest error found (approximately double the mean). This suggests that a sweet-spot of where the target should be placed in the tracking volume. . . . . . . . . . . . . . . . .

4.5 Deviations along optical axis for a single straight line cutting trial, measured using m-mode OCT. . . . . . . . . . . . . . . . . .

4.6 Algorithm steps for osteotomy experiment. a) Scapula with penscription. b) SL surface. c) User selected points. d) Mapping to flattened image space. e) Image dilation. f) Gaussian blurred. g) Sobel edge detection filter. h) Nearest neighbour growth algorithm. i) Mapping back to image space. j) Skeletonization via averaging. k) Post multi-order least squares polynomial fit. 1) Normal vectors generated using principal component analysis.

4.7 a) Patient scan of a frontal ethmoidal sinusectomy. b) Red outline showing trajectory. c) Trace of osteotomy outline onto porcine scapula. d) Ablated trajectory. . . 70 
5.1 System diagram. The fiber laser system was built directly into the sample arm of the OCT based depth ranging system, allowing for real-time monitoring of the kerf bottom using m-mode imaging whilst ablating. . . . . . . . . . . . 76

5.2 Results of ablation times for $0.5 \mathrm{~mm}$ depth with no graphite chromophore applied and with graphite chromophore applied to bone surface. We see a dramatic improvement in mean and variance of the time it takes to reach the target depth from when the laser was activated. Y-axis is in log scale. . . . . . . . . . . 78

5.3 Effects of nitrogen flow during percussion ablation of medium density fiber board (MDF) for different exposure times. . . . . . . . . . . . . . . . . 80

5.4 Mean etch rate as a function of exposure time and flow rate. Etch rate is quite high during the smallest exposure window and highest nitrogen gas flow. Etch rate is seen to increase as a function of flow rate specifically, but an increase flow does not linearly translate to increase in etch rate. . . . . . . . . . . . .

5.5 Time progression of depth measured via inline m-mode OCT, as a function of flow rate. 30SCFH is shown to most dramatically improve ablation. 70SCFH is shown to improve slope initial ablation progression, just before $200 \mathrm{~ms}$. . . . . . . . . . .

6.1 Methods for ablation and OCT beam combination. Image a) shows the most common method, using a dichroic mirror to combine the high-powered ablation beam with relatively low-powered OCT beam. The wavelength-specific transmissivity of the dichroic element prevents high-powered ablation light from following the beam path of the OCT system. b) shows a method that involves a fiber laser to be built directly into the sample arm of the OCT system, as proposed in [9]. . . . . . .

6.2 System diagram. The 7-DOF manipulator carries optical payload (with objective optics) over target. Feedback of axial position over target's surface is measured in real-time via OCT and correction is sent to the robot over high-speed serial communication. The two elements highlighted in dashed lines (objective optics and delivery fiber) show where either of the two method of beam combination explained in figure 6.1 would take place. . . . . . . . . . . . . . . . .

6.3 Anatomy of a focused gaussian beam. Depth of focus denotes planes along optical axis at which beam area is twice that of the area at beam waist. $L_{d} / 2$ denotes Rayleigh length. It is typically desirable to maintain the specimen surface at the beam waist. . . . . . . . . . . . . . . . . . . .

6.4 a) Schematic of fiber based swept-source OCT system. b) Point spread function (PSF) of OCT system. Axial resolution $=9 \mu \mathrm{m} . \ldots \ldots \ldots$

6.5 Elimination of ambiguity in de-focusing direction. The plot represents the fringe signal post-Fourier transform. The peak represents the position of the target surface along the optical axis. The frequency is directly proportional to the optical path difference, therefore position of the peak along the frequency axis represents the absolute position of the surface along the optical axis. . . . . . . . . . . . 90

6.6 Response of system correction to step change in the axial direction. . . . . . . . . 92 
6.7 Schematic of slope progression experiment. $\theta$ represents the angles that the surface was positioned to. . . . . . . . . . . . . . . . . . 92

6.8 Phantom experiments summarizing tracking error versus linear speed and slope. Panels a), b) and c) show m-mode tracking error at 10, 20 and 45 degree surface incline, respectively. Blue line demarcates Rayleigh length. d) shows mean of tracking error experiments. . . . . . . . . . . . . . 93

6.9 a) Diagram showing axial adaptation on a skull phantom target. Experiment is executed over the transverse medial suture. Total axial height change is $7 \mathrm{~cm}$ over the $10 \mathrm{~cm}$ linear translation. b) and c) Axial tracking error results over transverse suture of the skull phantom for $0.5 \mathrm{~mm} / \mathrm{s}$ and $1.0 \mathrm{~mm} / \mathrm{s}$, respectively. Red lines represent Rayleigh lengths. . . . . . . . . . . . . . . . . . . . . . . . . 94 


\section{List of Tables}

3.1 Parameters of fiber laser. . . . . . . . . . . . . . . . . . . . . 32

3.2 Results summarizing drilling depth accuracy experiments on MDF phantom. . . . . 43

3.3 Results summarizing drilling depth accuracy experiments porcine scapula specimens. 44

5.1 Summary of fiber laser parameters. . . . . . . . . . . . . . . . . . 77

5.2 Summary of OCT system parameters. . . . . . . . . . . . . . . . . 77 


\section{List of Abbreviations}

\begin{tabular}{|c|c|}
\hline Abbreviation & Meaning \\
\hline AOT & Advanced Osteotomy Tools \\
\hline $\mathrm{APC}$ & Angled Polished Connector \\
\hline BRW & Brown-Roberts-Wells \\
\hline CAD & Computer Aided Design \\
\hline CARLO & Computer Assisted Robot-guided Laser Osteotome \\
\hline $\mathrm{CCD}$ & Charge-Coupled Device \\
\hline CSF & Cerebrospinal Fluid \\
\hline $\mathrm{CT}$ & Computed Tomography \\
\hline DBS & Deep Brain Stimulation \\
\hline $\mathrm{DC}$ & Direct Current \\
\hline DFT & Direct Fourier Transform \\
\hline DLR & Deutsches Zentrum für Luft- und Raumfahrt \\
\hline DMD & Digital Micromirror Device \\
\hline EPFL & Ecole Polytechnique Federale de Lausanne \\
\hline FDA & Food and Drug Administration \\
\hline FD-OCT & Fourier Domain Optical Coherence Tomography \\
\hline FWHM & Full-Width, Half-Maximum \\
\hline GMAW & Gas Metal Arc Welding \\
\hline GUI & Graphical User Interface \\
\hline HAZ & Heat Affected Zone \\
\hline HR-FBG & High Reflectivity Fiber Bragg Grating \\
\hline ICG & Indocyanine Green \\
\hline INN & Iterative Nearest Neighbour \\
\hline IR & Infrared \\
\hline KIT & Karlsruhe Institute of Technology \\
\hline LMA & Large Mode Area \\
\hline LWR & Light Weight Robot \\
\hline MARS & Motor Assisted Robotic Stereotaxy System \\
\hline MDF & Medium Density Fiber Board \\
\hline MEMS & Microelectromechanical System \\
\hline MEP & Motor Evoked Potential \\
\hline MZI & Mach-Zehnder Interferometer \\
\hline NA & Numerical Aperture \\
\hline OC-FBG & Output Coupling Fiber Bragg Grating \\
\hline OCT & Optical Coherence Tomography \\
\hline OPL & Optical Path Length \\
\hline OR & Operating Room \\
\hline OTI & Optical Tomographic Imaging \\
\hline
\end{tabular}

Continued on next page 
Table 0.1 - Continued from previous page

\begin{tabular}{|ll|}
\hline Abbreviation & Meaning \\
PCA & Principle Component Analysis \\
POI & Pixel of Interest \\
PSF & Point Spread Function \\
RFTC & Radio Frequency Thermocoagulation \\
SCFH & Standard Cubic Feet Per Hour \\
SEEG & Stereotactic Electroencephelography \\
SL & Structured Light \\
SMF & Single Mode Fiber \\
SSEP & Somatosensory Evoked Potential \\
SS-OCT & Swept-Source Optical Coherence Tomography \\
TCP & Tool Center Point \\
TFBG & Tilted Fiber Bragg Grating \\
TMS & Transmagnetic Stimulation \\
TURP & Transurethral Resection of the Prostate \\
USP & Ultrashort Pulse \\
\hline
\end{tabular}




\section{Chapter 1}

\section{Introduction}

$\mathrm{O}$

STEOTOMY, or the cutting and removal of bone, is a fundamental part of surgical practice. Modification and excision of osseous skeletal tissue is required in countless modern surgical procedures, though the concept of bone cutting is far from avant-garde. Archaeological evidence shows that osteotomy of the skull has been performed by and on humans since the mesolithic period [10]. The first recorded mention of osteotomy technique was made by Hippocrates circa 415 BC [11], where he described osteotomy via traumatic fracture - luckily, techniques have progressed a long way from this. Despite a well-patinaed history, the physics of osteotomy has remained relatively unchanged: simple machines, such as wedges, levers and abrasive tools forcefully interact with the bone for splitting, cutting, etching, removal, and grinding. These primitive interaction modalities have remained ubiquitous irregardless of surgical specialty. Arguably, the most significant technological change that came about in osteotomy practice was with the introduction of motor-actuated tools, starting in 1908 by Bryant $[12,13]$, and to a lesser extent with the introduction of piezoelectric actuation in 2004 by Vercellotti [14, 15]. To date, dominant osteotomy techniques still require tool-tissue contact, and still rely on the surgeon's kinaesthetic sense (their sense of touch/feel) to be executed successfully - this is not too dissimilar from carpentry. The "tools of the trade" resemble many of those found in standard hardware stores for purposes of cutting bulk building materials (eg. wood, metal, etc.). As discouraging of a notion as this may be, one can take comfort in knowing that these tools, however primitive, are used to save lives in the hands of highly skilled surgeons. Still, higher precision, greater control and 
faster execution is always demanded from even the most advanced of technologies, be it medical or other. Improvement in osteotomy technology may lead to increased cutting precision and accuracy, cleaner incisions, less peripheral tissue damage and shorter surgery times. The development of tools that can achieve increased spatial accuracy in cutting, have real-time control of incision depth, and improve the consistency in cut quality is imperative for decreasing healing times and improving patient outcomes [16].

Cranial osteotomy, known as craniotomy, is performed when the surgeon requires access to the intracranial space for many types of brain surgeries. Currently, power-actuated tools for craniotomy offer little in the way intelligent safety design. Perforators, which are multi-sized bits used for cutting circular holes into the human cranium, use a simple clutch mechanism as a basic mechanical safety control system. The perpendicular force applied by the surgeon during drilling engages the clutch and allows the bladed tip to spin; once penetration of the inner skull table occurs, the sudden drop in axial force disengages the clutch and tip rotation ceases [17]. As one can imagine, this mechanism is highly sensitive to angle: any deviation from the drill being held perfectly perpendicular to the skull surface reduces reliability of timely clutch disengagement. Side cutting saws used for opening skulls, known as craniotomes, only rely on a small piece of metal angled over the distal end of the spinning cutting blade to shield the dura from accidental puncture [18]. This can limit angular positioning, increase friction during lateral motion, and potentially exacerbate already damaged dura via inadvertant snagging. Both of these very common tools still rely heavily on the surgeon for safe operation. This means that the tools have no active, engineered intelligence for soft tissue damage mitigation. The most significant determining factor of whether accidental dural penetration occurs or not is ultimately the surgeon's individual skill. Although these simple mechanisms offer some (albeit minimal) amount of safety, iatrogenic damage to distal cranial soft tissue during skull opening does occur. According to a study by Engelhardt et al., nearly a third of craniotomies resulted in dural tears, with a significant amount of these being multi-layer tears [19]. Dural tears such as these increase the risk of infection and cerebral spinal fluid (CSF) leaks. Accidental tears have an increased probability of occurring due to dural layers 
sticking to the inside of the skull. This is more prevalent in elderly patients, patients with hyperostosis frontalis, and/or patients with extracerebral lesions such as meningiomas [20]. As well, risk of iatrogenic damage can occur when patients have bone-related maladies, such as, but not limited to osteoporosis, osteomalacia and osteosarcoma. Dural tears can also be acutely dangerous if any of the dural venous sinuses are breached. These channels exist within the layers of the dura mater and are responsible for draining venous blood and CSF. Injury to these structures can result in massive bleeding and/or thrombosis. Recognizing how commonly this procedure is performed, the potential medical impact of accidental dural tears can be significant for the population of neurosurgery patients.

The use of lasers in medicine has been proposed almost as early as the days of Maiman and his pioneering ruby laser [21]. Numerous studies have been performed to determine how lasers can be used to cut tissue, photonically treat diseases and diagnose pathologies. The development of medical lasers promoted a certain romantic, futuristic promise of extra-human surgical precision; so much so that science fiction has largely seemed to do away with bladed scalpels in exchange for laser ones (eg. Star Trek, Final Fantasy, etc). Promises of similar magnitude can be said to have been made in the field of medical robotics. Koh's pioneering medical robotics work in the late 1980's was thought to mark the beginning of the automated surgery era [22]. So have these technologies failed in their promises? Did they not live up to their fervor? The answer may be that the timelines were over-imagined, not the technologies themselves. Lasers and robotics do have the potential to revolutionize surgery the way they have the manufacturing sector. However, each technology has it's own progression-impeading hinderances to surmount before ubiquitous operating room (OR) adoption can happen. Besides the obvious technical hurdles, what remains a challenge is the harmonious integration of these technologies into current surgical work-flow, not only for the surgeons, but for nurses and all other OR personnel. Marvels of engineering ingenuity run the risk of becoming storage relics if they are too difficult to use, maintain, or slow down the entire procedure without much added benefit. If a simple scalpel takes less than a minute to prepare and performs the same task to an sufficient degree, then it's hard to make the case for complex 
laser and robotic systems that provide only marginal medical benefit. Feats of medical engineering must not be overshadowed by poor user-interface design, long setup time or incompatibility with the OR environment; the proposition of benefit must be clear and worthwhile.

The purpose of this dissertation is to contribute technical advancements in the design of a system that can perform high-precision laser osteotomies to within sub-millimeter distances of full penetration. The surgeon will be able to intuitively and quickly define trajectories without additional planning necessary than the current practice. Above the existing paradigm, the system will aim to make osteotomies safer and faster without the trade-offs of work-flow disruption or steep user learning curves. The ultimate goal of such system would be to out-perform standard surgical saws in speed, safety and precision.

Robot assisted laser osteotomy is no trivial feat. No attempts that have been made in the past have successfully transitioned into clinical use thus far. It is the belief of the author that the reason for this is because issues of depth control and ease-of-use have not been properly addressed. Besides the clinical potential of the system that will result from this $\mathrm{PhD}$ dissertation, other contributions include:

- Design of a novel ablation depth controlled all-fiber laser apparatus;

- A study of bone ablation using inert gas assistance with varying parameters;

- A demonstration of the efficacy in thermal ablation of bone using a topically applied chromophore;

- A novel method for defining arbitrary cutting trajectories on the surface of boney tissue;

- A novel method to maintain beam waist of focused gaussian beam onto target surface.

Most of the individual technologies that are leveraged throughout this dissertation have been developed to their current maturity relatively recently. Therefore, it seems that these technologies have converged at what may be the correct time for the work outlined in this dissertation to take place successfully. The goal of this work is to impact the practice of neurosurgery and to increase the standard of care for patients. This dissertation is rooted in practicality and as such, it is 
hoped that this research will successfully make the bench to bed-side transition one day, eventually rendering the surgical drill a tool of the past.

\subsection{Outline}

This dissertation is organized as follows:

- Chapter 2 gives a background of osteotomy, laser-tissue interaction fundamentals, laser osteotomy, Optical Coherence Tomography (OCT) fundamentals and brief histories on neurosurgical robotics and the use of OCT for ablation monitoring;

- Chapter 3 details the construction and experimental results of the novel OCT + fiber laser system that was designed for this work. Two addendums are included in this chapter: 1) details of an alternate structure that uses bulk optics to integrate the fiber laser into the sample arm of the OCT system and 2) details of an alternate method for k-clock sample trigger delay, which is discussed in detail in this chapter;

- Chapter 4 details the novel algorithm developed for penscriptive visual servoing of the robotic manipulator. Experimental results are presented and discussed to demonstrate system viability;

- Chapter 5 presents experiments and results of the study conducted to optimize laser bone ablation using graphite as a topical chromophore and a nitrogen assist gas jet;

- Chapter 6 demonstrates the use of OCT for real-time refocusing and correction of drift along the optical axis of a robotic end effector used for laser osteotomy;

- Conclusions are made and areas of future research are discussed in Chapter 7. 


\section{Chapter 2}

\section{Background}

ERVOUS tissue is typically less robust to damage than muscle, bone, cartilage and many
other forms of tissue found throughout the body. This is due to nervous tissue's severe lack of regenerative capabilities when compared to most other forms of somatic tissue $[23,24]$. Therefore, surgery in and around neural anatomy is a process that requires precision and patience to avoid iatrogenic damage. Loss of organ functions, sensory loss, paralysis and death can occur upon significant damage to the spine and brain.

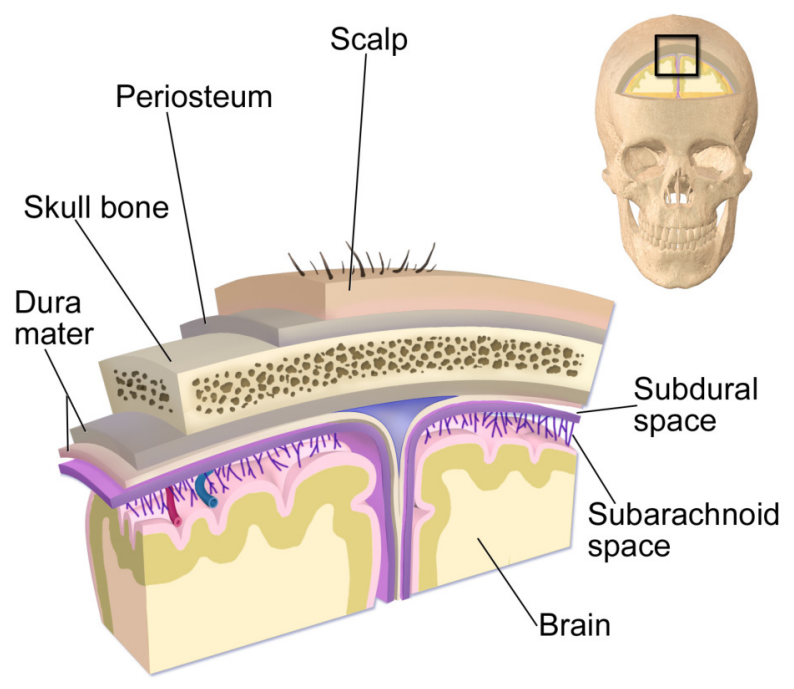

Figure 2.1: Layers covering the brain [1].

Figure 2 shows the layers of tissue above the brain surface. Typically during most cranial 
procedures that require an open surgical incision and osteotomy, the soft tissue layers of the scalp and periostium are first peeled back to reveal the skull surface. Underneath the skull, the brain is encased in a sack of water-tight soft tissue known as the dura mater. Inside, the brain is held in neutral bouyant suspension in cerebrospinal fluid (CSF). The endostium (not shown) is a layer of soft tissue attached to the outer surface of the inner layer of cortical bone just before the dura.

The skull itself is composed of three distinict layers of bone. Both superficial layers of the skull bone are composed of cortical or compact bone, which is composed of densely packed layers of cylindrically shaped clusters known as osteons (shown in figure 2(a)).

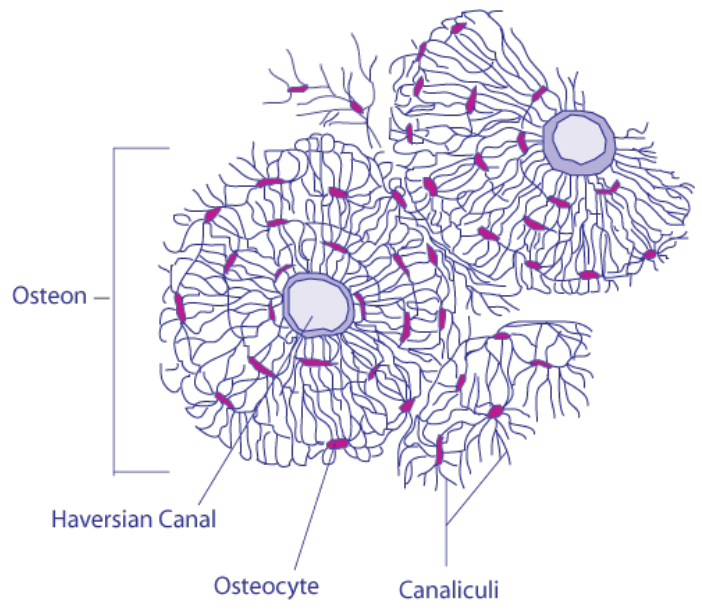

(a) Cross section of an osteon

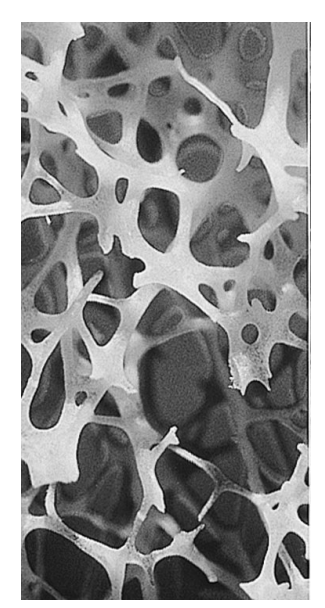

(b) Trabeculae

Figure 2.2: Fundamental units of the two types of bone (cortical [2] and trabecular [3]).

Sandwiched in the middle of the two cortical layers resides the trabecular or cancellous bone. This layer composed of strut-shaped soft tissue units known as trebeulae. The trabecular bone layer is typically where bone related metabolic activity occurs and as such, tends to be heavily vascularized compared to cortical bone. Figure 2(b) shows a microscopic image of trabecular bone.

The spinal column consists of a series of irregularly shaped vertebral bones that extend from the base of the skull down to the pelvic girdle. The spine is separated into three distinct regions: 7 cervical vertebrae (C1-C7), 12 thoracic vertebrae (T1-T12) and 5 lumbar vertebrae (L1-L5). The individually articulating vertebrae terminate at the sacrum, which consists of five fused vertebrae. 


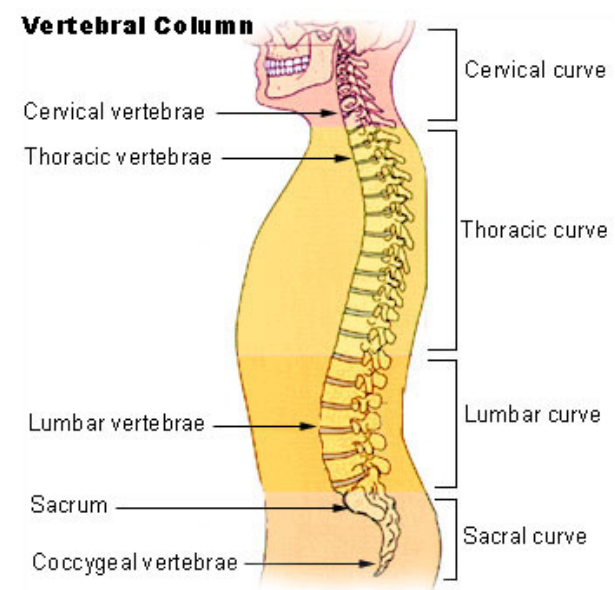

(a) The spinal cloumn

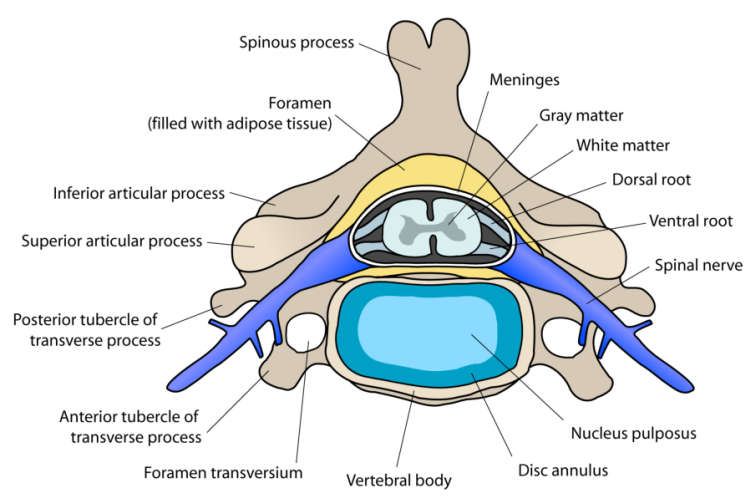

(b) Cross section of a vertebrae

Figure 2.3: Details of the spinal column [4] and vertebrae [5].

Inferior to the sacrum, attached via a partially flexible joint sits the 4 fused vertebrae of the coccyx. The central hole of each vertebral disk, known as the vertebral foramen line up to form the spinal canal, a protective boney channel that runs the entire length of the spinal column. This channel encases the spinal cord. Similar to the brain, the spinal cord is wrapped in a water-tight covering known as the meninges. Inside the spinal canal, protective adipose tissue cushion the encased spinal cord from direct contact with the internal foraminal surfaces. Anterior to the foramen sits the vertebral body, which forms the bulk of the disk. The posterior surface of the vertebrae has the prominent transverse process, a boney spike jutting out posteriorly. To each side of that is the lamina and the facet joints. Two more prominent spikes, the transverse processes, stick out of either side of the disk.

\subsection{Osteotomy in spine and cranial surgery}

Osteotomy can be classified in to three categories: excision, reparative and for approach [27]. Excision osteotomy is any procedure which requires the removal of pathological bone, be it infected, cancerous or spurious ossification. Reparative osteotomy requires modification of the bone for purposes of anatomical correction. Osteotomy for approach purposes is required when osseous tissue 


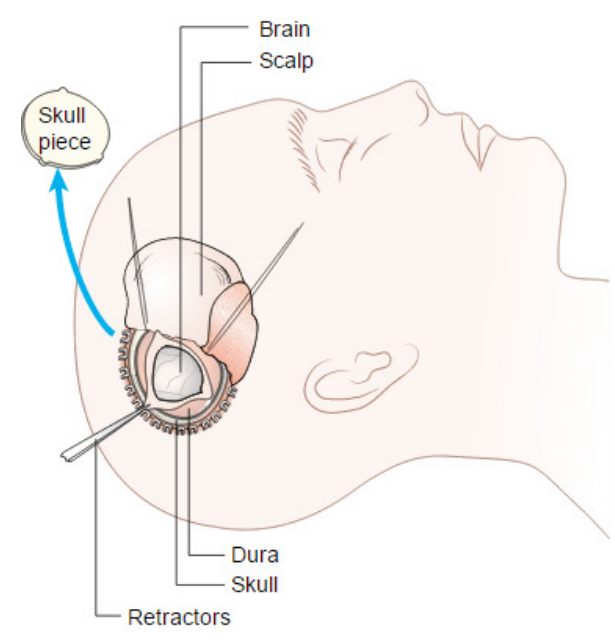

(a) Diagram of a craniotomy [25]

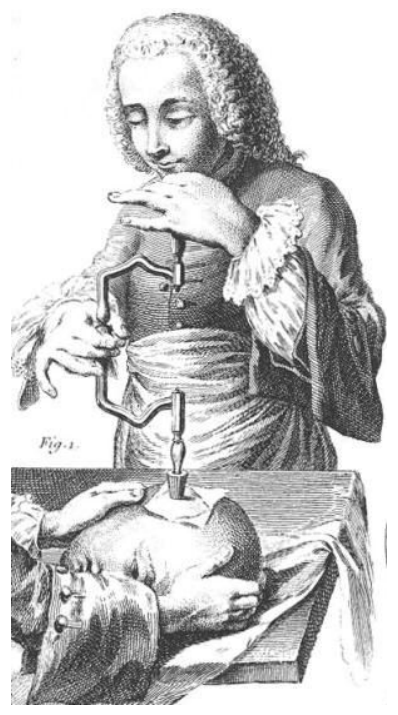

(b) Trepanation [26]

Figure 2.4: Common examples of cranial osteotomies.

needs to removed in order to gain access to a region of interest. Sections of cut bone are sometimes (but not always) returned to the original removal site at the end of the surgery. A common example of this would be removal of a skull flap (craniotomy) to gain access to the intracranial space. Tools used to perform osteotomy are considerably similar to those found in carpentry. Larger osteotomies are typically performed with drills (motorized and hand-powered), saws (manual, reciprocating and circular), chisels and osteotomes. When more precision is required, tools such as rongeurs, forceps, scissors, files, rasps and gouges are used. Regardless of which tool is used, the application of some combination of mechanical forces to hard tissue remains the dominant physical mechanism. Because of this, risk of iatrogenic mechanical damage to surrounding soft tissue, such as the cutting burr plunging into the soft cranial tissue is a real risk [28, 29, 30]. This risk can easily be actualize into morbidity if the surrounding soft tissue is especially sensitive; this is the case in neurosurgery.

Certain large cuts are quite common in brain surgery. As mentioned earlier, a craniotomy, shown in figure 2.1, is a common cut that is made to remove a flap of bone to gain access to the soft tissue inside the intracranial space. A burr hole, or trepanation, is made in the skull when a large cut 
is not needed such as in the case of cranial biopsies. Both are done with powered and/or manual drills.

Bone cutting in the spinal column offers different challenges to the surgeon, given the tighter space and irregularity of the shapes of the bones. Usually drills with finer tips are used to mill through boney surfaces, compared to the larger drill heads and more plunge-like drilling motions with regards to the skull. A laminectomy is common procedure where the surgeon cuts away the lamina of the vertebrae. This is done to accommodate insertion of surgical hardware (i.e. screws, rods, plates, etc) or to relieve stenosis of the vertebral foramen. A laminoplasty does involve full penetration of at least one lamina. Instead, a lamina is "scored" so that it can be folded to increase foraminal space.

\subsection{Laser osteotomy: techniques and challenges in modern med- ical uptake}

Despite numerous investigations over the last few decades exploring the use of laser bone ablation, no standard practices exist of laser osteotomy in most types of surgery. In fact, the use of lasers in surgery is generally quite limited. FDA approved procedures for soft tissue excision in a handful of surgeries does exist (i.e. TURP surgery for the prostate) but is still limited. Approved procedures involving bone (but not necessarily osteotomy) include thermal ablation of vertebral facet nerves, and disc decompressions to reduce disc herniation [31]. Limited use of laser osteotomy is approved in dentistry. For example the Biolase WaterLase iPlus is a hand operated dental laser device that is FDA approved for soft and limited hard tissue work inside a patient's mouth. Currently there are no FDA approved devices that use laser technology to perform osteotomy on the spine or skull.

So far, however, most bone ablation studies have been conducted on animal models and exvivo human tissue specimens. Laser osteotomy studies have been conducted in various aspects of osteotomy, including peripheral tissue damage [32, 7], post-operative recovery [33, 34, 35, 36, 37], and hemostatis (especially in oral osteotomies) [37, 38, 39]. As such, lasers show a great potential in improving osteotomy over current surgical drills. 


\subsection{Laser interaction with osseous tissue}

T ASER light is a multi-purpose tool in the context of surgery and medicine. Laser light can be 1 used in low beam-energy applications such as innocuous diagnostic imaging or high beamenergy applications for therapeutic methods, such as drug activation and tissue ablation. For purposes of osteotomy, high beam-energy with high energy density (focused beam) is primarily used to etch away bone material. Interaction of laser light with any biological tissue is primarily governed by the absorption and reflection characteristics of the tissue. According to Niemz, the process of ablation starts with heat generation through laser light interaction with the tissue based on laser and the tissue's optical parameters, the heat is then transported through the tissue, which subsequently leads to the various heat-induced effects [7].

For ablation and therapeutics, absorption tends to be the dominant parameter. In tissue, light attenuation due to absorption can be described by Beer-Lambert's Law:

$$
I(z)=I_{o} e^{-\mu_{a} z}
$$

where $I(z)$ is the intensity at depth $z$ along the optical axis, $\mu_{a}$ is the coefficient of absorption, which is a function of the wavelength and material. Scattering behaviour may also be taken into account to better understand the interaction dynamics of light as it enters tissue. The scattering coefficient, $\mu_{s}$ describes the scattering behaviour of light similar to $\mu_{a}$. Given this, the mean free path $L_{m p}$, (typically) in $\mathrm{cm}$, is naturally

$$
L_{m p}=\frac{1}{\mu_{t}}
$$

where

$$
\mu_{t}=\mu_{s}+\mu_{a}
$$

This describes the mean length that a photon can travel within a medium before an interaction event (absorption or scattering) is likely to occur. For OCT imaging, $L_{m p}$ is important because only photons that experience coherent backscatter, that is photons that have experienced no absorption, 
minimal scattering events (total length travelled being less than the coherence length of the source), and are able to travel back directly into sample arm, retain a coherent phase relation with the photons travelling in the reference arm so that interference fringes can be produced (see section 2.5). This can be further understood by the anisotropy factor $g$, which is a value between -1 and 1 that describes the likelihood of forward scatter during a photon scattering event. $g$ is used to modify $\mu_{s}$ such that

$$
\mu_{s}^{\prime}=(1-g) \mu_{s}
$$

Where $\mu_{s}^{\prime}$ is known as the reduced scattering coefficient. $g=1$ describes purely forward scattering (no scattering at all), $g=-1$ describes purely backscattering, and $g=0$ describes isotropic scattering, that is, scattering with equal likelihood in any direction. This is contrasted to the photons used for etching material, where absorption and depth of penetration (and by extension forward scattering and anisotropy) are dominant factors; coherence is not necessary to consider for the ablation source.

According to Neimz, we can use the absorption coefficient from Equation 2.3 to understand the heat deposition $S$ in a local cylindrical volume as a function of time [7]:

$$
S(r, z, t)=\mu_{a} I(r, z, t)
$$

where $I$ is a the electric field intensity of the incident light, $r$ is the radial distance perpendicular to the optical axis, $z$ is the distance travelled along the optical axis and $t$ is time. This absorbed energy is transferred into heat and phononic vibration in the tissue lattice, and part of the heat energy does get transferred into thermoelastic expansion. For the purposes of this dissertation, we only consider the light-to-heat transfer portion of the interaction.

To provide a cursory understanding of heat flow through a material, we introduce thermal relaxation time, $\tau_{\text {therm }}$ which is defined as the time it takes for the temperature of a volume of material to reach $50 \%$ of the maximum temperature, above a baseline. It can be related to $\mu_{a}$ with the following relation: 


$$
\frac{1}{\mu_{a}}=\sqrt{4 k \tau_{\text {therm }}},
$$

Where $k$ is the thermal diffusivity (in $\mathrm{m}^{2} / \mathrm{s}$ ). Isolating $\tau_{\text {therm }}$, we get:

$$
\tau_{\text {therm }}=\frac{\rho c_{p}}{4 K \mu_{a}^{2}}
$$

where $\rho$ is the volumetric mass density $\left(\right.$ in $\left.\mathrm{kg} / \mathrm{m}^{3}\right), c_{p}$ is the specific heat capacity of the material (in $\mathrm{J} / \mathrm{kg} \cdot \mathrm{K}$ ) and $K$ is the thermal conductivity (in $\mathrm{W} / \mathrm{m} \cdot \mathrm{K}$ ). This concept is important to understand since it can allow laser ablation parameters, such as pulse duration and exposure time, to be selected such that thermal breakdown can be caused to occur in the target tissue volume. According to Niemz, if the exposure time is greater than $\tau_{\text {them }}$, heat can diffuse into a multiple of $\frac{1}{\mu_{a}}$ into the tissue.

We see from equations 2.2 and 2.7 that light diffusion into tissue is heavily dependant on $\mu_{t}$, and total heat deposition and thermal relaxation are dominated by $\mu_{a}$. Since most forms of tissue have significant water content, the absorption characteristics of water are understood to have a significant and even primary role in laser tissue interaction. Figure 2.3 shows the absorption spectrum for water. It can be seen from the figure that water is highly absorbing in the UV region and beyond $10 \mu \mathrm{m}$, with a sharp spike at near $3 \mu \mathrm{m}$. A valley is seen in and around the visible band; this understood to be responsible for many of the photochemical reactions necessary to life and evolution (ie. photosynthesis, optical vision, vitamin D synthesis, etc.).

Bone is a complex matrix that is optimized to withstand loads and forces. Human bone, by composition, is 50-60\% hydroxyapatite, a hard, inorganic material composed of calcium phosphate crystals. Approximately $22 \%$ of bone consists of organics, which is primarily collagen and a small amount of protein. Approximately 15-20\% of bone consists of water. It is vital to understand the absorption characteristics of the molecular constituents of bone to ascertain a better understanding of it's interaction with laser light. 


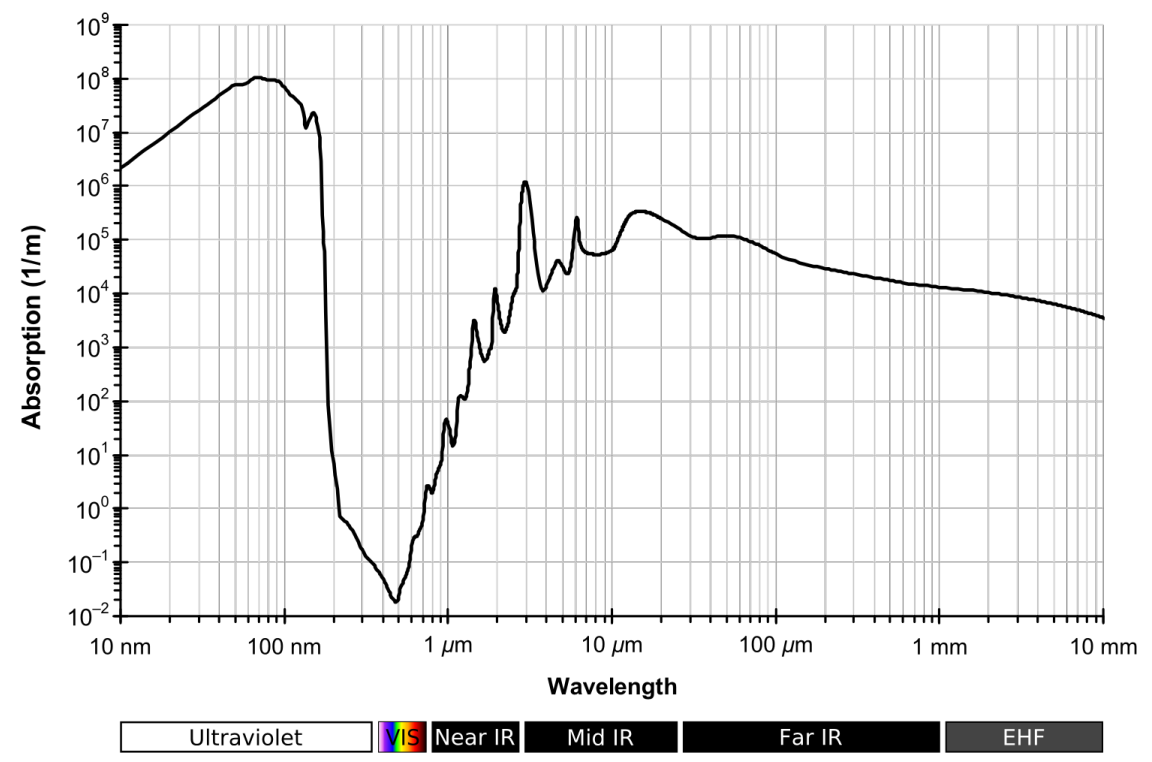

Figure 2.5: Absorption spectrum of water [6].

\section{Mechanisms of interaction}

To understand what happens locally to a volume of tissue during application of light energy, we must introduce the Arrhenius Damage Integral, which describes the thermal injury inflicted onto the tissue based on the rates of reactions of the reactants with the application of energy above a threshold, the activation energy $E_{a}$. The equation, with temperature-time dependance, is described as follows [40]:

$$
\Omega(T, t)=\int_{0}^{\tau} A e^{\frac{-E_{a}}{R T(t)}} d t
$$

where $T(t)$ is the local tissue temperature with respect to time (in Kelvin), $R$ is the molar gas constant. A, referred to as the frequency factor or pre-exponential factor, is a quantitative description of the frequency of molecular collisions that are specifically conducive for the specific reaction to take place; the units are $\mathrm{s}^{-1}$.

Depending on pulse duration, wavelength and energy density, a variety of ablative interaction mechanisms between a given material and incident laser light can occur at the atomic level. Interaction mechanisms/behaviour vary as a function of power density and pulse duration/exposure 
time. The main mechanisms of interaction are [7]:

- Photochemical Interaction

- Thermal Interaction

- Photoablation

- Plasma Induced Ablation

- Photodisruption

In the context of this dissertation, the mechanisms of main importance are thermal interaction and plasma induced ablation.

Thermal interaction, as suggested by the name involves localized heating of a specific volume of tissue. In order to etch away tissue, the level of heating has to produce a vaporization effect for material to be carried away. Figure 2.3 shows the effect thermal ablation has on the direct heated volume and surrounding tissue. As seen, layers in the tissue form according to the amount and duration of deposited heat energy with respect to depth. Just underneath the pocket of where the tissue had been vaporized, a thin layer of carbonized tissue is formed. Vaporization and carbonization occur when the tissue is heated to $100^{\circ} \mathrm{C}$ and greater (boiling point of water at sea level). A layer of coagulated tissue underneath. This occurs when the tissue has reached a temperature of approximately $60^{\circ} \mathrm{C}$. The heat damage that both these layers exhibit are irreversible; this tissue has been destroyed. The hyperthermic layer which forms between the coagulated zone and unaffected tissue can heal, as not enough heat energy has been deposited into this layer as to cause cell death. This occurs when the tissue has been heated to approximately $45^{\circ} \mathrm{C}$; not high enough to denature proteins and collagen. Nuss et al. conducted a study of bone ablation and found that the zone of damage did not extend to more than $15 \mu \mathrm{m}$ beyond the edge of the ablation site using an Nd:YAG laser $(1064 \mathrm{~nm})$ for radiant exposures ranging from 8 to $27 \mathrm{~J} / \mathrm{cm}^{2}$ [41].

Plasma induced ablation occurs when the electric field component of the incident laser light has a high enough intensity to cause optical breakdown of the material in the localized volume. When a certain threshold intensity is reached, the incident field induces ionization inside the target volume. 


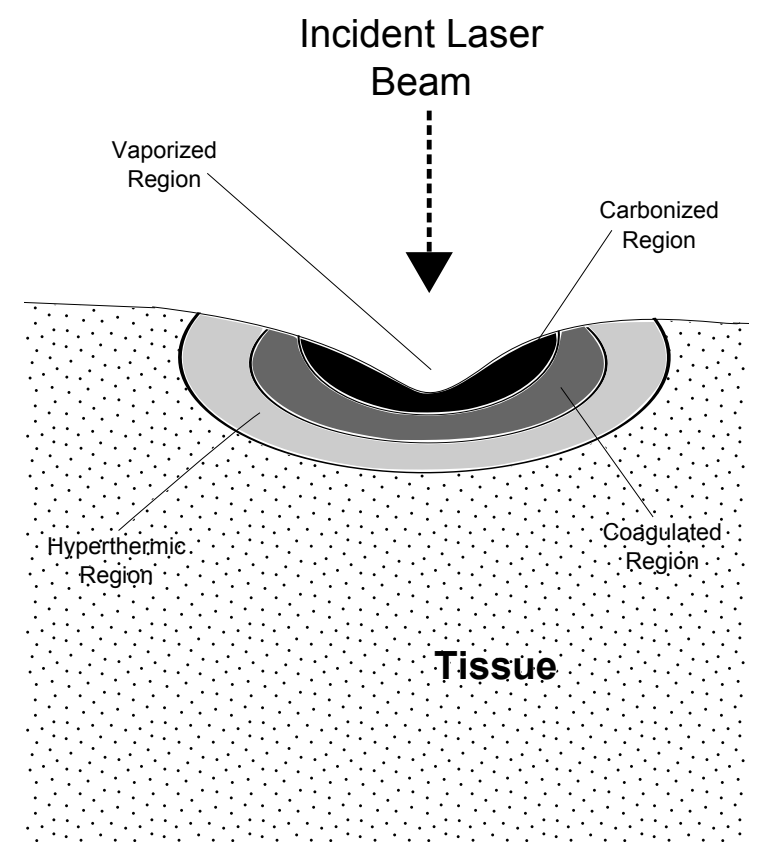

Figure 2.6: Heat affected zone (HAZ) during laser tissue ablation [7, 8].

This threshold is dependent on the intramolecular forces within the material. It is important to note that optical breakdown is a special case of the more general dielectric breakdown. Thresholds for plasma formation are heavily dependent on laser pulse duration and irradiance. Generally, most solids and fluids experience optical breakdown and intensities around $10^{11} \mathrm{~W} / \mathrm{cm}^{2}$.

\subsection{Use of assist gas in laser material processing}

Use of assist gas jets in laser material processing has been known to be beneficial in applications involving cutting of bulk materials. Gas jets, usually co-axial with the beam, help by:

- Clearing away melt, dross, debris and plasma so that laser light can interact with the bottom of the channel that is cut into the material, known as the kerf;

- Keeping workpiece cool, reducing extent of the HAZ and sometimes reducing laser energy necessary to produce similar cutting results; 
- Evacuating oxygen around the ablation site to reduce flare-ups (in the case of inert gases);

- Forcing spatter away from the optics.

Assist gas jets were shown to dramatically improve etch rate up to a certain point, after which no improvement and even slight detriment was shown to occur [42]. It was realized that this unexpected decrease in cutting efficiency was found to be a result of shock waves induced as a result of high-pressure incident gas jets for applications that use high flow rates and pressures [43]. In the case of ablation for osteotomy purposes, nitrogen would be the gas of choice given it's bio-compatibility and availability in standard ORs. As well, medically relevant pressures and jet velocities for cutting would be much lower than the efficiency boundaries discovered by Gabzdyl [42].

\subsection{OCT fundamentals}

\subsubsection{Interference of light}

Optical Coherence Tomography (OCT) can be understood by understanding the principles governing light interference. Two coherent light beams of a single wavelength with intensities $I_{1}$ and $I_{2}$, respectively, occupying the same region of space will yield a resulting intensity $I$ described by the interference equation [44]:

$$
I=I_{1}+I_{2}+2 \sqrt{I_{1} I_{2}} \cos \varphi,
$$

where $\varphi$ is the phase difference between $I_{1}$ and $I_{2}$. It can be shown through the inherent oscillatory behaviour of the cosine function that in every full cycle of $\varphi$ from 0 to $2 \pi$, the resulting intensity $I$ will fluctuate between 0 and $2 I_{o}$, where $I_{o}=I_{1}+I_{2}$.

Figure 2.7 shows a simple Michelson interferometer. Light from a coherent light source (usually a laser) is split into two distinct beams of a fixed ratio at the beam splitter. Each beam travels toward a mirror, where it is reflected back. The two reflected beams are then recombined at the beam splitter, resulting in a steady-state intensity. Equation 2.9 describes the behaviour of the interferometer when viewed from from the position of "Observer", shown at the bottom of the figure. 


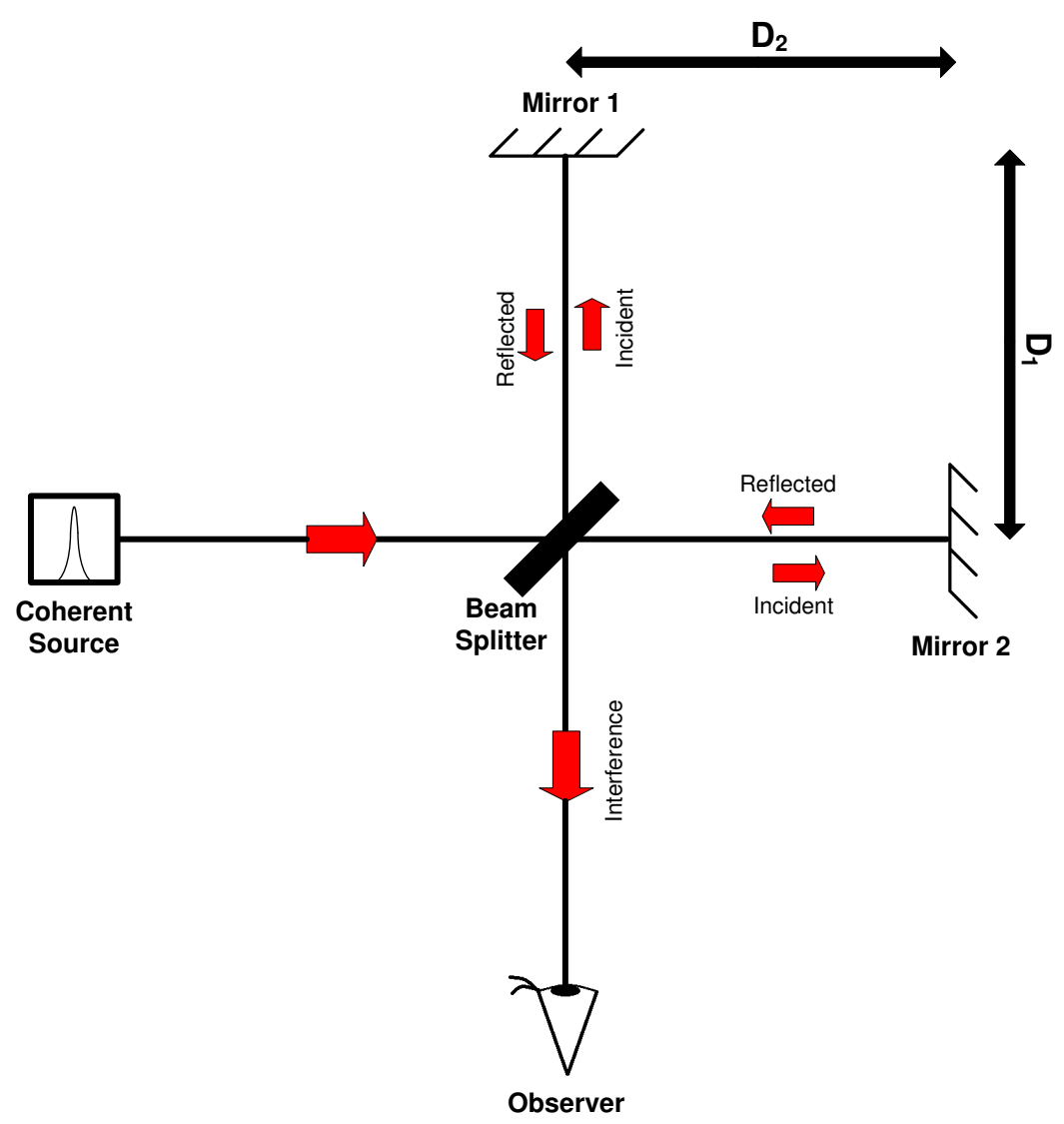

Figure 2.7: A Michelson interferometer.

The resulting phase difference $\varphi$ that governs the behaviour of the interference term of the equation is directly proportional to the difference between $D_{1}$ and $D_{2}$, which are the distances between the beam splitter and each respective mirror; this is known as the path difference. If the path difference is increased and/or decreased with respect to time (i.e. keeping one mirror fixed and linearly translating the other along the optical axis), a series of oscillations in intensity will be seen at the observer end; this is known as fringes. Analyzing these fringes will result in information about the path difference.

It is important to note that Equation 2.9 makes the simplification that the interference of the two light beams will oscillate indefinitely as the path difference grows without bound. This is not the case with real coherent sources because interference can only take place when the path difference stays within a limited length known as the coherence length, which is solely a property of the source. 


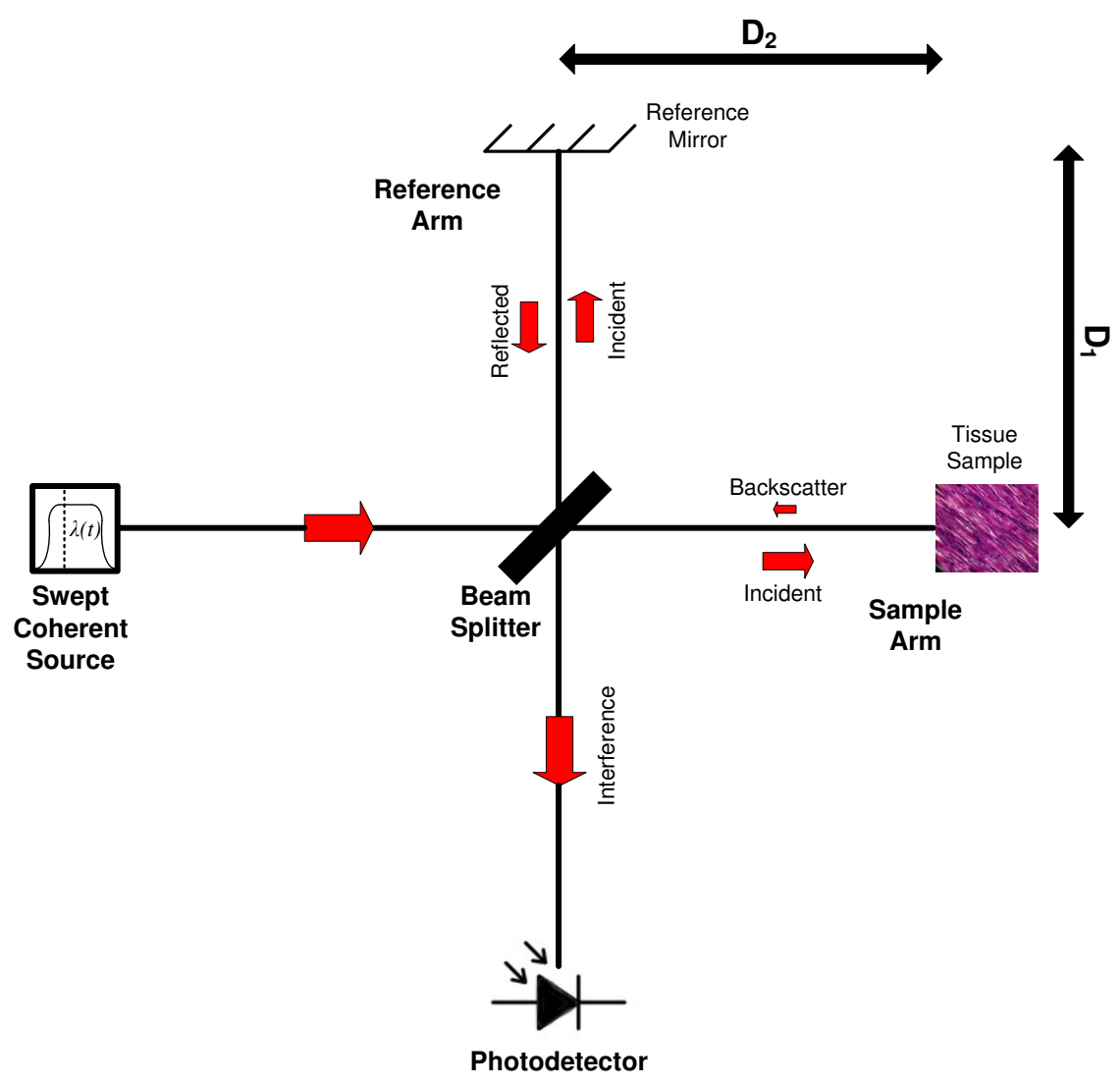

Figure 2.8: Functional diagram of a swept source OCT system.

\subsubsection{Swept source OCT}

Swept Source Optical Coherence Tomography (SS-OCT) takes advantage of the wavelength dependent depth discrimination of backscattered light that occurs when coherent light is incidentally directed onto layered media, such as tissue. Figure 2.8 shows a schematic of an SS-OCT system. When compared to Figure 2.7, it is important to note some differences. First, the light source is changed to one where the output's center wavelength is time dependent. Another difference is that one of the arms of the interferometer is now terminated at a tissue sample rather than a mirror. This means that the portion of backscattered light sent back to the beam splitter is largely attenuated. Lastly, a photodiode is placed at the observer end of the interferometer so that the intensity resulting from the fringe signal is realized as a photocurrent.

The following equation describes the photocurrent induced at the detector of the SS-OCT scheme in fgure $2.8[45,46]$ : 


$$
I_{\text {photo }}=R\left[\left(P_{\text {ref }}+P_{\text {samp }} \int r^{2}(z) d z\right)+2\left(\sqrt{P_{\text {ref }} P_{\text {samp }}} \int r(z) \Gamma(z) \cos (2 k(t) z+\Phi(z)) d z\right],\right.
$$

where:

- $R$ is the responsivity parameter of the photodiode;

- $P_{r e f}$ is the power in the reference arm;

- $P_{\text {samp }}$ is the power in the sample arm;

- $r(z)$ is the depth dependent reflectivity profile of the sample;

- $k(t)$ Describes the time-varying output of the swept source;

- $\Gamma(z)$, known as the coherence function describes the coherence of the swept source;

- $\Phi(z)$ is the depth dependent phase profile of the sample.

Analyzing the equation further, it can be shown that the DC response of the photodiode is represented in isolation by:

$$
I_{D C}=R\left(P_{\text {ref }}+P_{\text {samp }} \int r^{2}(z) d z\right),
$$

and the $\mathrm{AC}$ term, which describes the interference behaviour, is represented by:

$$
I_{A C}=2 R \sqrt{P_{\text {ref }} P_{\text {samp }}} \int r(z) \Gamma(z) \cos (2 k(t) z+\Phi(z)) d z,
$$

In SS-OCT, a single axial (depth) scan at a single point on the sample happens with each sweep of the full bandwidth of the swept source; this typically happens at kilohertz speeds. The resultant interference-induced photocurrent signal is then acquired and a Discrete Fourier Transform (DFT) is performed. The resulting signal is known as an A-line. The transverse resolution of each A-line, $\delta_{x}$, is dependent on the numerical aperture of the focusing optics and the center wavelength of the line-width, described by the following equation [46]: 


$$
\delta_{x}=0.37 \frac{\lambda_{0}}{N A},
$$

The axial (depth) resolution is characterized by the coherence length of the system, approximated by the following equation [45]:

$$
\delta_{z}=l_{c}=\frac{2 \ln (2) \lambda_{0}^{2}}{\pi \Delta \lambda},
$$

where $\lambda_{0}$ is the line width center frequency and $\Delta \lambda$ is the $3 \mathrm{~dB}$ bandwidth of the laser spectrum. Since depth information is inherent in each A-line, a two-dimensional image can be acquired if the incident beam is scanned in the transverse direction. If the beam is scanned along the third axis, a three-dimensional volume can be acquired.

The sweeping frequency of the source determines how many A-lines are able to be acquired per second; thus it is the determining factor of frame rate and overall system speed (assuming sufficiently fast acquisition electronics).

\subsection{A brief history of neurosurgical robots}

Sakaguchi reported a proof of concept robot that was to be used for percutaneous nephrostomy [47]; this was one of the first reported papers published in the realm of surgical robotics. The first recorded use of a robot in an operating theatre was in 1985 in British Colombia, Canada [48]. The Arthrobot was used for joint positioning curing orthopaedic knee surgery. Shortly after, at the Memorial Medical Centre in Long Beach, CA. A PUMA robot was used in performing a sterotactic biopsy on a brain lesion of a 52-year old patient [22]. The robot was used to hold a guide for the biopsy needle which was to be inserted into the patient's brain. After the end-effector of the robot was positioned in the desired pose, the robot was locked in position and the power was removed. The robot was shown to have $0.05 \mathrm{~mm}$ position accuracy. This was also the first example of a robot used in neurosurgery.

In 1991, researchers at the University of Toronto and Hospital for Sick Children used a PUMA 200 robot in one of the first applications of robotics in paediatric neurosurgery [49]. The robot 
was used to hold a cylindrical retractor measuring $7.5 \mathrm{~cm}$ in length and $2 \mathrm{~cm}$ in diameter. This was done so to assist the surgeons in removing astrocytic brain tumours from 5 separate paediatric patients. The robot and the BRW (Brown-Robert-Wells) stereotactic head frame were both bolted on to the same platform to keep everything rigid. The result was that the robot was able to hold the retractor in the pre-operatively planned trajectory. Even though the PUMA 200 was known to have an accuracy of $0.05 \mathrm{~mm}$, observed error was cited to be as high as $2 \mathrm{~mm}$. Patient morbidity was low and no patient mortality was reported. Similar work in robotics use in stereotactic surgery was was reported in 1995 by Glauser et al. at the Ecole Polytechnique Federale de Lausanne (EPFL) [50]. The MINERVA system was used to perform biopsies of lesions on 8 patients were performed using this system; 6 were deemed successful while one had to be aborted due to physical constraints and the other turned out to be a histological mis-diagnosis. The procedures in this study were executed under real-time CT guidance.

Another PUMA robot, this time the 260 model, was adapted for use in the very first spine surgery application by a group in Gernoble, France in 1992 [51]. Sautot et al. used the robot to intraoperatively position and hold a laser guide for drilling holes into the vertebrae of a plastic spine model. Such holes are commonly made in procedures such as spinal fixations to anchor fixation devices, such as pedicle screws, into vertebrae. The goal of this project was to use the robot to help the surgeon accurately follow the optimal, pre-planned trajectory during surgery so that the risk of cortical bone-breach and/or spinal column and peripheral nerve laceration is reduced. Besides claiming sub-millimeter accuracy, no quantified value was given. However, the experimentally made holes (done percutaneously) were said to have been executed without deviation from the preoperatively planned trajectories.

Starting in the late 1990 's, a tele-operated manipulator-based robot for frameless stereotactic neurosurgery was developed by the Robotics Institute of Beijing University of Aeronautics and Astronautics and the Navy General Hospital in Beijing, China. This culminated in the CAS-BH5 system, the 5th generation prototype. It was used in 2007 and was evaluated on 10 patients in the city of Yan'an in Shaanxi province, while the surgeon was stationed in Beijing (approximately $1300 \mathrm{~km}$ away). The study showed that after a 12 month follow up, 9 of the 10 patients showed 
neurological improvement and no complications were reported to be found [52]. In 2014, the CAS-R-2 system, an earlier prototype from 2001 [53], was used on 7 patients for the implantation of radio frequency thermocoagulation (RFTC) electrodes in the bilateral mesial temporal lobes. Of this study, four patients achieved Engel Classifcaton level I (no more disabling seizures) [54].

Ortmaier proposed the KineMedic in 2005, a 7-DOF manipulator based robotic arm guided by a infrared fiducial-based navigation camera system (BrainLab) for pedicle screw placement [55]. The project was done through the Institute of Robotics and Mechantronics at the German Aerospace Center (Deutsches Zentrum r Luft- und Raumfahrt e.V., or DLR).

A master-slave manipulator device was developed by Mitsuishi et al. at the University of Tokyo in 2012 to perform neurosurgical anastomosis. $0.3 \mathrm{~mm}$ and $0.5 \mathrm{~mm}$ blood vessels phantoms were successfully anastomosed, but no clinical trials or animal trials were reported [56].

In 2012, researchers at Hitachi Ltd. and Shinshu University School of Medicine (Nagano Prefecture, Japan) developed the NeuRobot, a tele-operated neuroendoscopic robot with three micromanipulators. The group successfully demonstrated fenestration of the third ventricular floor and septum pellucidum, third ventriculostomy, and biopsy of the choroid plexus and thalamus on cadaveric head models [57]. In 2011 and 2012, Heinig demonstrated a stereotaxy robot, dubbed MARS (Motor Assisted Robotic Stereotaxy System) and reported a mean positioning error of $0.60 \mathrm{~mm}$ with a mean deviation and mean repeatability of $0.23 \mathrm{~mm}$ and $0.06 \mathrm{~mm}$ respectively. A clinical trial of a tumor biopsy was successfully conducted $[58,59,60]$. Arata et al. developed a neurosurgery-specific master console to guide a robot during a procedure. This console was developed using a 5-DOF parallel linkage platform with pneumatic sensors that were able to detect suction and contact forces [61]. In 2014, Grau et al. used a transcranial magnetic stimulation (TMS) robot to transmit information between the brains of two subjects [62]. The robot, the Axilum Robotics TMS Robot, is a CE and Health Canada approved system.

In 2010, researchers at Karlsruhe Institute of Technology (KIT) developed a the world's first robotic laser osteotomy system. Two iterations of the system were initially developed (for craniotomy [14] and cochleostomy [63]), the first with the carbon dioxide laser payload being carried by a 6-DOF RX90B-CR, the second with a 7-DOF KUKA LWR4+. This project developed a a 
complete workflow, beginning with image acquisition and continuing with optimized trajectory planning and finally execution on ex-vivo animal bone specimens. Imaging was done via preoperative CT scans. Marking and cutting experiments using simple trajectories (lines, cuboids, triangles) were carried out on sample tissue and showed accurate results with no greater than $0.56 \mathrm{~mm}$ deviation. This work was done for the ultimate purpose of precision guided cochleostomy surgery. To this end, the work is still continuing with inline OCT imaging for high-precision tracking and depth monitoring [64, 65, 66, 67, 68, 69, 70, 71, 72, 73].

Advanced Osteotomy Tools AG (AOT) is a company based in Basel, Switzerland with a device similar to that similar to the Karlsruhe researchers. Named CARLO (Computer Assisted and Robot-guided Laser Osteotome), the system has been demonstrated to cut simple geometries. The system consists of a KUKA LBR IIWA robot carrying what appears to be a laser payload with "opto-acoustic" depth monitoring devices for depth control during ablation. The system is in development for use in oral and maxillofacial surgery $[74,75]$.

\subsubsection{FDA approved neurosurgical robots}

At the time of writing of this dissertation, only 6 robots are approved for neurosurgery:

-SpineAssit, Mazor Robotics

-Renaissance, Mazor Robotics

-Mazor X, Mazor Robotics

-ROSA, MedTech

-Neuromate, Renishaw

-Excelsius GPS, Globus Medical

It must be noted that these robots do allow for osteotomy in the very strict sense of the word, insofar as they allow for percutaneous holes to be drilled into boney structures for minimally invasive and/or stereotactic procedures. However, these systems only allow for single holes to be drilled at a time and do not perform the osteotomy autonomously; all holes are drilled by the surgeon, using 
a conventional bone drill via an end effector-mounted sleeve, positioned along the hole trajectory for drill bit guidance. Bone flaps and burr holes for direct access to the intracranial space cannot be realized using any of these systems.

Mazor Robotics, a company based in Caesarea, Israel, has solely focused their product line thus far on percutaneous pedicle screw insertion. The SpineAssist robot was their first to receive FDA approval, in 2004. The robot is a 6-DOF miniature parallel manipulator hexapod designed to guide the surgeon's tools along a pre-determined trajectory to allow for optimal pedicle screw placement [76]. Comparable in size to a soft drink can, the robot sits outside the body on a rigid linear track running along patient's spine, to which it is firmly afixed. The frame is placed over the vertebral level of interest and intraoperative fluoroscopy (anteroposterior and oblique views) is used to register the spatial placement of the frame with the pre-op CT scan. The robot is then attached to the frame and software commands the robot to the desired attitude over the vertebrae in order to position the sleeve along the desired screw trajectory. Mazor introduced the Renaissance, an update to the SpineAssist, in 2011 [77]. The Renaissance was reported to have an improved human interface, improved registration procedures and stereotactic brain surgery capabilities. The Mazor X system consists of a cart-mounted 6-DOF robotic manipulator with a much larger working volume compared to the two other robots in the Mazor line up. However, much like the other two systems, the robot is rigidly attached to the patient anatomy during setup. The system has the capability of rendering a 3D map of the surgical field surface using a laser scanner system [78]. Khan et al. reported in 2018 a study where the Mazor X system was used to place 75 screws in twenty patients. 74 of the 75 screws were classified as Ravi scale grade I (no breach or deviation from planned path [79]) and the mean time for insertion of each screw was 3.6 minutes. This group also reported that the learning curve was minimal for the use of this system [80].

The Excelsius GPS by Globus Medical (Audubon, Pennsylvania, USA) is a relative newcomer to the spinal fixation market. The system received FDA approval in August 2017 [81]. This system also uses a manipulator to place a sleeve in the path of the pedicle screw to guide the surgeon's tools. A unique feature of this robot is that it can measure forces in real time that are imparted 
on the surgical site and issue a warning if these forces reach a danger zone. As well, unlike the previous systems, the Excelcius GPS does not have to be rigidly connected to the patient's anatomy. To date, only a single study can be found reporting on the system's efficacy on two patients. This study showed a screw tip deviation of $2.1 \mathrm{~mm}$ and a mean angular offset of 2.4 degrees for 8 screws. $88 \%$ of the screws inserted were evaluated to be grade A and the rest grade B using the GertzbeinRobbins scale [82].

The Renishaw Neuromate is a multi-DOF manipulator-based systems used for stereotacitc surgery. The manipulator-based robot acts as a sterotatctic instrument holder to help surgeons guide and secure tools and instruments such as biopsy needs and deep-brain stimulation (DBS) electrodes. Just as with the previous robots, the patient must be rigidly fixed to the base of the robotic system for accurate registration and guidance. According to Yasin et al., the stereotactic approach using the Neuromate has comparable diagnostic capabilities and complication rates to traditional stereotactic biopsies [83]. In 2013, Abhinav et al. reported on a study where the Neuromate was used to place intracerebral, deep brain electrodes (stereotactic electroencephalography, or $S E E G$ ) for the treatment of focal epilepsy. It was found that even though the system allows for accurate implantation and that no major complications were reported, the mean time of electrode placement was increased from 3.1 hours to 5.6 hours. The authors state that a source of error was in the application of the robotic system rather than it's accuracy capabilities. The authors specifically mentioned application of the frame as a source of error [84]. The ROSA, by Medtech (Montpellier, France, now Zimmer Biomet, Indiana, USA) is a robot similar to the Neuromate and Mazor $\mathrm{X}$. The ROSA is capable of both stereotactic brain procedures and assistance in pedicle screw insertion. Chatillon et al. used the ROSA system for implantation of 80 electrodes in 13 patients and compared that to the manual placement of 37 electrodes in 6 patients. It was found that using the ROSA offered a $2.5 \mathrm{~mm}$ increase in target error accuracy and a $2.6 \mathrm{~mm}$ improvement in axial deviation [85]. 


\section{A note on the da Vinci robot}

Even though it's not used or neurosurgery, the Da Vinci robotic surgical system by Intuitive Surgical (Sunnyvale, California, USA) is the most well-known and one of the most widely used surgical robots in the world. The system consists of a surgeon console and a tele-operated bedside robot. It has three arms that are used to hold multiple tools including endoscopes, scalpels, scissors, etc. These arms are introduced inside the patient's body via minimally invasive ports. In 2000, the system became one of the first to be FDA approved (for laparoscopic surgery at the time [86]). At this time, the system is able to perform bariatric, urological, gynaecological, lung and general surgical procedures [87, 88]. According to [86], there are over $4400 \mathrm{Da}$ Vinci systems in hospitals worldwide and over 43000 surgeons trained on them. As of 2017, over 5 million MIS surgeries have been performed using the Da Vinci system. The Da Vinci is mentioned in this dissertation because it is seen as a pioneering surgical robotic system, and any advances in robotic surgery in general can be seen to have directly or indirectly benefited form Intuitive Surgical's work.

\subsection{Objectives}

The objective of this doctoral dissertation is to make a novel contribution to the fields of medical robotics and laser osteotomy. Inspired by the work of Burgner [14], we propose that the methods developed in this work can be used to further the technology related to robotic laser osteotomy, for which the ultimate goal is to reduce or eliminate methods of bone cutting via mechanical contact.

This dissertation has the following sub-objectives for contrubuting to the field of biophotonics and surgical robotics:

- The integration of a high-powered fiber laser with coaxial inline OCT for depth guidance through an all-fiber beam path;

- Trajectory generation and end-effector orientation of a 7-DOF robotic manipulator using simple penscription and structured light imaging.

- Enhancement of bone ablation at $1064 \mathrm{~nm}$ through the use of a topical chromophore (graphite); 
- Enhancement of bone ablation at $1064 \mathrm{~nm}$ using a coaxial nitrogen assist gas jet;

- The use of OCT for real time, sub-millimeter axial correction of the end-effector of a robotic manipulator in order to maintain beam focus; 


\section{Chapter 3}

\section{Fiber Laser and Inline OCT Integration}

The work presented in this chapter resulted in the following journal publication [89]:

Jivraj, J., Chen, C., Huang, Y., Ramjist, J., Lu, Y., Vuong, B., Gu, X. and Yang, V.X., 2018. Smart laser osteotomy: integrating a pulsed $1064 \mathrm{~nm}$ fiber laser into the sample arm of a fiber optic 1310nm OCT system for ablation monitoring. Biomedical Optics Express, 9(12), pp.6374-6387.

\subsection{Introduction}

As mentioned previously, the ability to monitor axial penetration during laser ablation into bone tissue is desirable because it can be used as a feedback mechanism for depth control. This way, the critical bone-endosteum interface, which demarcates the edge of the soft-tissue volume inside the neurocranium, can be approached with caution so as to not accidentally penetrate and damage the underlying dura and neural tissue. Without a feedback mechanism, a priori knowledge of ablation dynamics and assumptions about tissue homogeneity would need to be used to estimate removal depth per pulse of unit time $[90,91]$. This would lead to variability in ablation results and poor adaptability to different tissue compositions.

Chapter 2 explained that the current paradigm to stop a mechanical bone drill from full breach into the dural layer is by the use of a cam-connected cutting bur bit. High magnification cameras 
have been used to view the surface of the cut during ablation, but this is inherently limited due to the lack of direct sub-surface information available [92].

In this chapter we explore the combination of OCT with a high-powered ablation laser for feedback control of bone ablation. OCT provides the inherent advantages of high-speed, sub-surface imaging, as well as the ability for the OCT laser light to be coaxially coupled to the machining beam.

\subsubsection{OCT coupled ablation for process visualization}

Ohmi et al [93, 94] demonstrated inline OCT monitoring of in-situ ablation of a human tooth using a Q-switched Nd:YAG laser at $1.06 \mu \mathrm{m}$ with a 10ns pulse width.The OCT and ablation laser were time-multiplexed across a dichroic mirror usng an electronic shutter. A certain number of pulses were fired onto the tooth, at which point the shutter was closed to block the ablation laser, and the OCT system was allowed to take a B-mode image. This process was repeated until the crater reached various depths. A study by Oh et al [95] achieved high-speed imaging of ablation using a swept-source OCT (SS-OCT) with an A-line rate of $115 \mathrm{kHz}$ and a video rate of 200 frames/s. Similar studies were done using FD-OCT systems combined with Nd:YAG and YAG lasers achieving 25 frame/s acquisition speeds [96, 94]. Torkian et. al. compared results of ablation crater metrics (width and depth) of porcine vocal cords between OCT and histology; no significant differences were found, thus proving OCT as a viable method of ablation depth measurement [97]. Although the authors of this study did not monitor ablation depth in real-time, the results prove that OCT is not only effecting in qualitative visualization of crater formation but is a viable tool to gather quantitative ablation related data - exactly what is needed to provide feedback in a closed loop controller. The first study showing in-situ, real time depth profiling during ablation was done by Webster et. al. [98]. The system described in the study used a single laser source for both ablation and imaging. A $1064 \mathrm{~nm}$ mode-locked fiber laser with repetition rate of $10 \mathrm{MHz}$ and pulse duration of 20ps was expanded, then split using a Michaelson interferometer into reference and sample beams. The sample beam was reflected off of a galvanometer-mounted mirror (used for 
beam positioning) and through a focusing objective lens. The light that was backscattered from the ablation process was combined with the reference beam at the interferometer and collected at the detection arm, where it was sent to a spectrometer. This study was able to demonstrate etch progression during ablation of stainless steel using M-mode OCT imaging (capturing OCT interferometric data at a single point rather than raster scanning the beam). The images generated were able to show when the ablation process sped up or slowed down. The A-line rate of the OCT system was $46 \mathrm{kHz}$, despite the source's $10 \mathrm{MHz}$ pulse repetition rate. A similar studies by the same group demonstrated full control using OCT-based metrology as the feedback mechanism [99]. Demonstration of successful OCT feedback used during hard tissue ablation was demonstrated in [100]. The authors were able to control the percussion drilling to within $50 \mu \mathrm{m}$ of full breach of cortical bone, thereby proving the potential of OCT in controlling laser ablation for surgical applications. Similar work using all-fiber designs have been carried out by Beaudette et al. [101, 102]. In both these studies, a coagulation laser was coupled to an OCT imaging system using a double-cladded fiber coupler, achieving essentially single-fiber beam delivery for coagulation and monitoring. The power scalability for this method is limited for two primary reasons: first, fiber couplers tend to have a low damage threshold (typically in the hundreds of milliwatts). According to Boulnois [8], ablation in the thermal regime during laser-tissue interaction roughly begins at a power densty of $10 \mathrm{~W} / \mathrm{cm}^{2}$ with an exposure time of roughly $10^{-3} \mathrm{~s}$; plasma mediated ablation requires higher powers at smaller exposure times. This demonstrates the need for coupling of higher power sources with OCT for effective etching of bone in a controlled manner. Second, the OCT system is left vulnerable to damage from the higher power coagulation light source. As well, in [101], a required beam-dump at port 4 of the coupler reduces overall efficiency of the entire system. In a 2015 study [9], we presented a precursor of this work where the integration of a $1 \mathrm{~kW}$ peak power/10W average power fiber laser and swept-source OCT system was achieved using a series of edge-pass, free space filters. M-mode progression of wood ablation was shown for up to $2 \mathrm{~mm}$ in ablation depth. This paper demonstrated the first time two laser systems of such high power difference were coupled into a single delivery fiber for use in an ablation-monitoring application. In 2017, a continuation of this work demonstrated a phenomenon where the peaks of the PSF were shown to 
Table 3.1: Parameters of fiber laser.

\begin{tabular}{|c|c|}
\hline Wavelength & $1064 \mathrm{~nm}$ \\
\hline Average Power & $3.35 \mathrm{~W}$ \\
\hline Peak Power & $419 \mathrm{~W}$ \\
\hline Pulse Duration & $160 \mathrm{~ns}$ \\
\hline Pulse Frequency & $50 \mathrm{kHz}$ \\
\hline
\end{tabular}

broaden as a function of optical path difference [103].

In this paper, we present an integrated high peak-power $1064 \mathrm{~nm}$ fiber laser within the sample arm of an OCT system at 1310nm center wavelength. This configuration allows for coaxial beam delivery without the use of a dichroic mirror at the output stage or any other free-space bulk optic elements for use in coupling. We also address and resolve the issue of depth-dependant PSF peak broadening.

\subsection{Methods}

\subsubsection{Ablation fiber laser}

The high powered fiber laser used for ablation is based on work by Lu et al. [104]. It consists of active Yb-doped fiber cavity (Nufern LMA-YDF-10/130-VIII) approximately 10m in length. The cavity is enclosed by Two fiber Bragg gratings (FBGs) etched onto mode-matched passive fiber (Nufern SM-GDF-10/125). One of the FBGs has high-reflectivity (HR-FBG) at the Bragg wavelength of $1064 \mathrm{~nm}(>99 \%)$, and the other is a lower reflectivity used for output coupling (OCFBG). Just after OC-FBG is another length of passive fiber with a cladding-mode pump stripper and APC connector terminating the output end. A 976nm fiber coupled laser diode (RealLight M976 $\pm 3-110-F 105 / 22-D 1)$ is used to pump the cavity via a $(2+1) x 1$ high power pump and signal fiber combiner (ITF MM021112CC1A). All fiber used has a core diameter of $10 \mu \mathrm{m}$; this means that the output ablation beam and OCT beam remain single-mode. Table 3.1 summarizes ablation laser characteristics. 


\subsubsection{Swept-source OCT}

An SMF-28 based fiber optic swept-source OCT (SS-OCT) system was used for this study. A MEMS swept-source (Santec HSL-20-50-S) with $\lambda_{c}=1310 \mathrm{~nm}$ and a $\Delta \lambda=110 \mathrm{~nm}$ and average output power of $18.5 \mathrm{~mW}$ was connected to a Michaleson interferometer of 50/50 coupling ratio. A Thorlabs BD415C balanced detector was used to detect the interferogram. The a-lne rate of the system was $50 \mathrm{kHz}$ with a $62 \%$ duty cycle. The k-sampling trigger was output directly from the MEMS unit.

\subsubsection{Fiber laser and OCT system coupling}

Beam coupling was achieved through the use of 3 tilted fiber Bragg gratings (TFBGs) placed in series between the output of the OCT sample arm and the signal port of the pump and signal combiner. The total attenuation at the Bragg wavelength of $1064 \mathrm{~nm}$ was approximately $20 \mathrm{~dB}$. Such a high attenuation was necessary since the peak power of the laser was 4 orders of magnitude higher than the swept-source average power; this means that light from the signal port of the combiner that is generated in the fiber laser could damage the OCT system components. Path matching between sample and reference arms of the OCT system was achieved by extending the reference arm roughly the same same distance as the length of the fiber laser; SMF-28 fiber was used.

\section{Bending loss and amplification}

It was found that the MEMS generated swept-source light $(1310 \mathrm{~nm})$ was being highly attenuated through integrated fiber laser sample arm. Splice losses and insertion losses due to passive components were found to be minimal. Further investigation revealed that bending of the passive constituent fibers of the fiber laser were responsible for the very high losses. The passive SMGDF-10/125 fiber has a numerical aperture (NA) of 0.086 at 1310nm, which is much lower than that of the SMF-28 (0.14) [105]. Bending of this passive large mode area (LMA) fiber allowed for guided mode leakage into the cladding and the subsequent ejection through the cladding mode pump stripper near the output pigtail. A series of bending experiments were carried out to deter- 


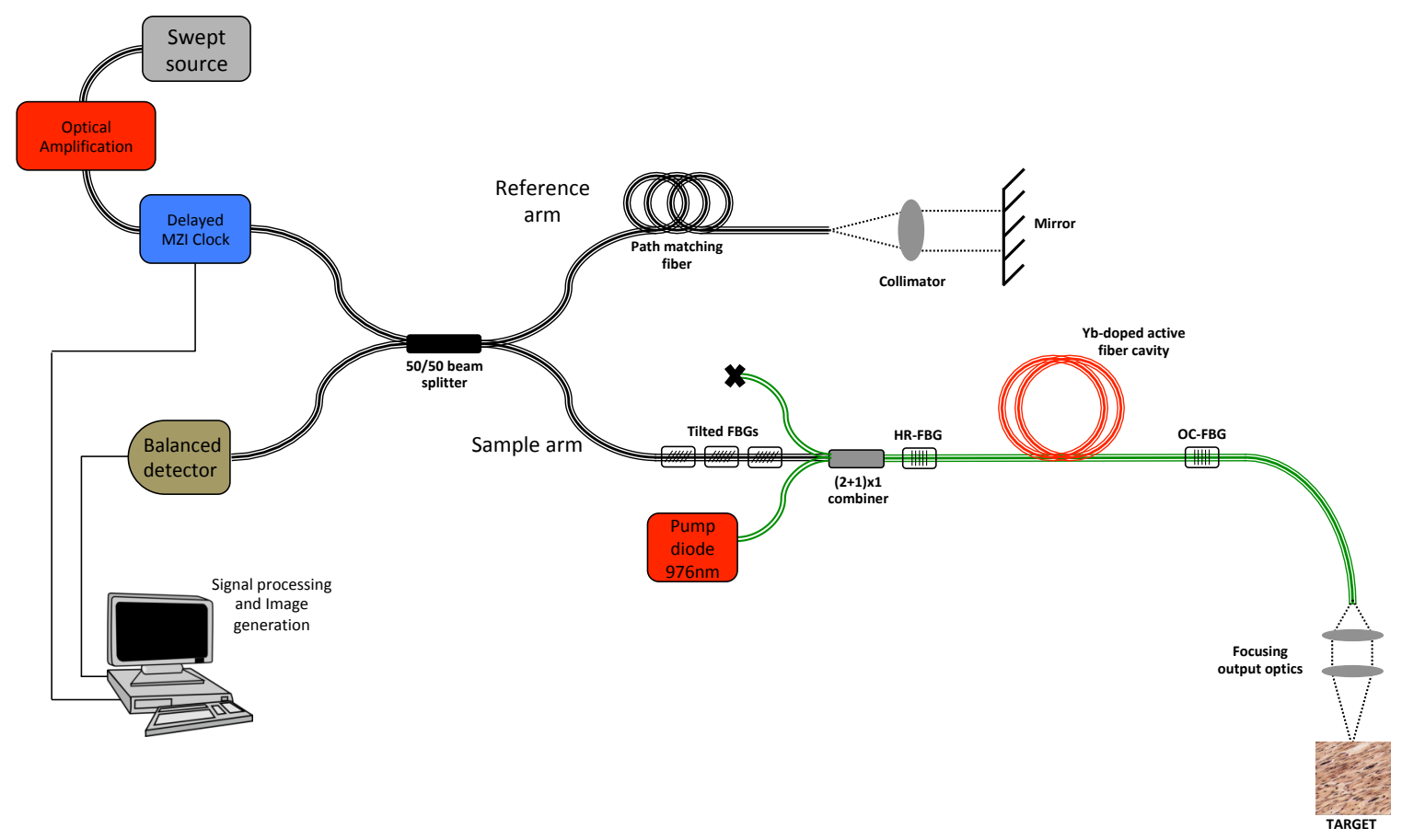

Figure 3.1: Schematic of the integrated OCT and fiber laser system. The entire fiber laser is built directly into the sample arm of the OCT system. Both systems are entirely fiber-based (aside from standard reference arm and focusing optics), allowing compact, robust packaging and elimination of dual-beam free-space alignment.(Reprinted with permission of the Optical Society of America)

mine loss severity due to this limitation; a straight length of fiber was bent along a semi-circular path with a varying radii. The swept-source generated light was launched through one end and measured out of the other. The results are summarized in figure 3.2. The figure shows that at a $15 \mathrm{~mm}$ bending diameter, the LMA passive fiber suffered an $9.7 \mathrm{~dB}$ attenuation of the OCT light; under he same conditions, SMF-28 exhibited a loss of only $0.15 \mathrm{~dB}$ for comparison.

An optical amplifier was used to increase the over-all OCT light power within the system in order to compensate for the relatively high losses through the integrates sample arm. A Covega BOA1017 was spliced between the swept-source and interferometer; the average power going into the interferometer was increased to which increased to $85 \mathrm{~mW}$. This raised the final average power of OCT swept-source laser light to $6.5 \mathrm{~mW}$, measured at the output pigtail of the fiber laser integrated sample arm. The measured power loss after the ablation laser is approximately $8.15 \mathrm{~dB}$ resulting in a measured sensitivity of $80 \mathrm{~dB}$; the theoretical sensitivity of $81.7 \mathrm{db}$ sensitivity. The expected sensitivity was measured to be $98 \mathrm{~dB}$ measured before the TFBGs and ablation fiber. Even though this attenuation is a limitation of this structure, the sensitivity is still more than adequate to provide 


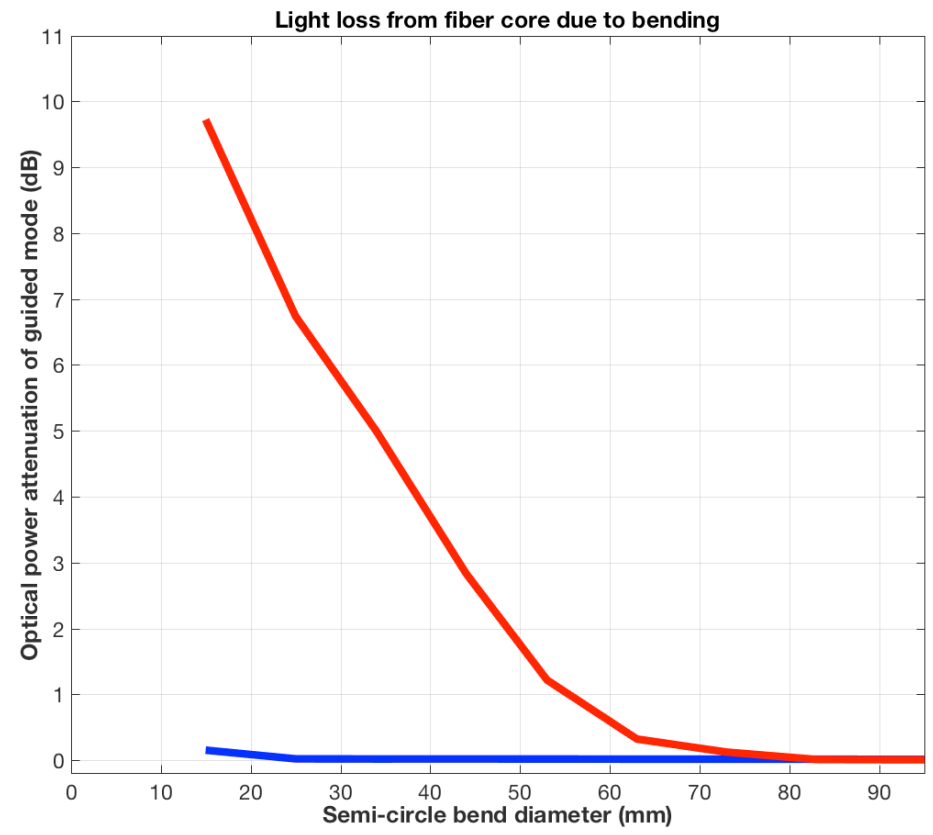

Figure 3.2: OCT swept-source light ejection from fiber core due to bending. For the data shown in this figure bends of 10 different diameters were performed, repeated three times and averaged. The red line represents the Nufern SM-GDF-10/125, and the blue line represents standard SMF-28 fiber. It is important to note how dramatic the ejection from the core of the Nufern SM-GDF-10/125 fiber is, up to approximately $53 \mathrm{~mm}$ of bending diameter, compared to SMF-28. (Reprinted with permission of the Optical Society of America)

clear and consistent surface detection. Strategies for mitigating further power lose due to output fiber bending are discussed at the end of this chapter.

\section{K-space re-sampling and point spread function}

The point spread function (PSF) can be defined as the impulse response of any imaging system; put simply, it demonstrates the smallest possible detail that an system can resolve. In OCT, the PSF is taken axially at varying depths by placing a mirror in sample arm to simulate a perfect scatter. This results in a sharp peak in the Fourier spectrum of the signal. A top-hat filter may be placed inside the sample arm as well to ensure that the interferogram is not saturated (this would cause the appearance of multiple false surfaces due to higher-order integer harmonics being present in the Fourier spectrum due to the change in frequency composition of a saturated or clipped signal). The $3 \mathrm{~dB}$ bandwidth of the peak is determined to be the resolving power of the system. The optical path difference OPD is increased by moving the mirror linearly along the optical axis, further away from the objective lens. The peak is measured at multiple distances to ensure that the resolution 


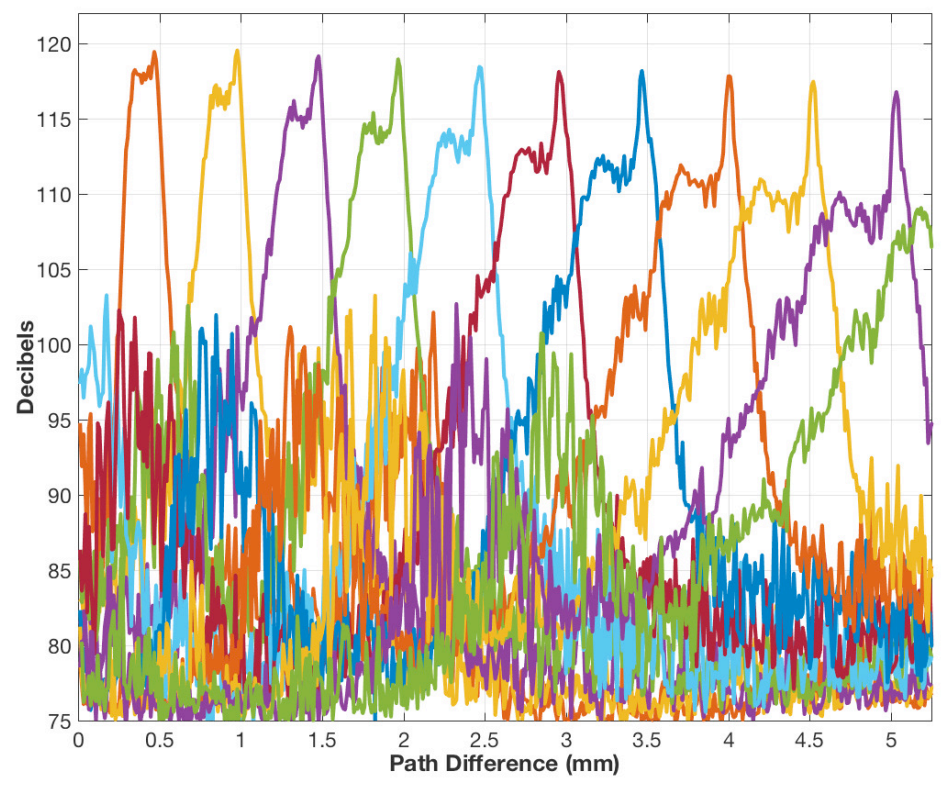

Figure 3.3: Point spread function (PSF) of system with no K-clock timing consideration. Optical path difference-dependant peak broadening is very evident due to un-delayed sampling trigger. (Reprinted with permission of the Optical Society of America)

stays relatively constant.

A phenomenon was seen in the PSF of the system described in this chapter that, to the knowledge of the author, has not been reported elsewhere in literature. It showed that the PSF was severely broadening as a function of optical path difference; this is shown in figure 3.3. The cause was determined to be the increased fiber length that the inline fiber laser added to the overall length of the OCT system. The total approximate length of the integrated fiber laser sample arm (as well as the reference arm) before the focusing optics was $23.1 \mathrm{~m}$; this excessive length introduced a significant increase in the round-trip time for the photons travelling through arm of the OCT system. For a single trip, this delay was estimated to be:

$$
\text { delay }=\frac{n(23.1)}{c}=112 n s
$$

where $n$ is the core refractive index of SMF-28 fiber (1.45205). The total estimated increase in round trip ( 2 single trips) time was $224 n s$. This delay was causing an equal electrical phase delay between the interferogram signal generated by the balanced detector and the k-clock sampling 


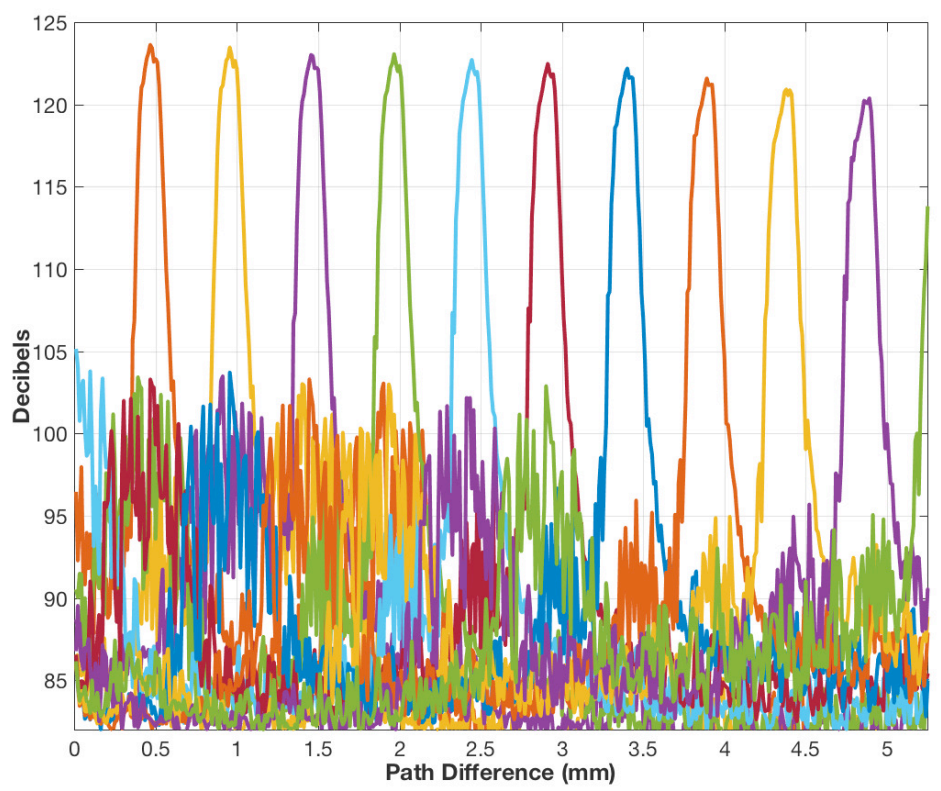

Figure 3.4: Point spread function (PSF) of the system with delayed MZI interferometer resampling clock to match round trip time of OCT light. Peak broadening is now approximately uniform due to high dispersion mismatch introduced by the inline fiber laser. (Reprinted with permission of the Optical Society of America)

trigger generated by the swept-source laser unit; which resulted in aliasing during sampling of the fringe signal.

To solve this it was determined that the k-clock sampling trigger needed to be delayed an equal amount. To achieve this, a custom Mach-Zehnder interferometer (MZI) based k-space resampling clock was constructed with approximately a total optical path length large enough to induce approximately the same delay and dispersion. The resulting PSF is shown in figure 3.4. As expected, all peaks are approximately evenly broadened. This is due to material dispersion mismatch caused by the sample and reference arms being constructed of mismatched fibers (SMF-28 in the reference arm and passive and active LMA fibers in the sample arm). Since this dispersion is constant, A $2^{\text {nd }}$ order software dispersion correction algorithm, similar to that found in [106] was used to correct this. The resulting PSF is shown in figure 3.5 with corresponding axial resolution values.

\subsubsection{A-line triggered ablation pulse phase delay}

Light generated in the fiber laser and travelling back through the combiner, into the OCT system is highly attenuated by the TFBGs placed in series. However, some leakage light still gets through and impinges on the OCT signal during A-line acquisition. The timing diagram demonstrating this is shown in figure 3.6. This impingement manifests itself as vertical stripes in the m-mode 


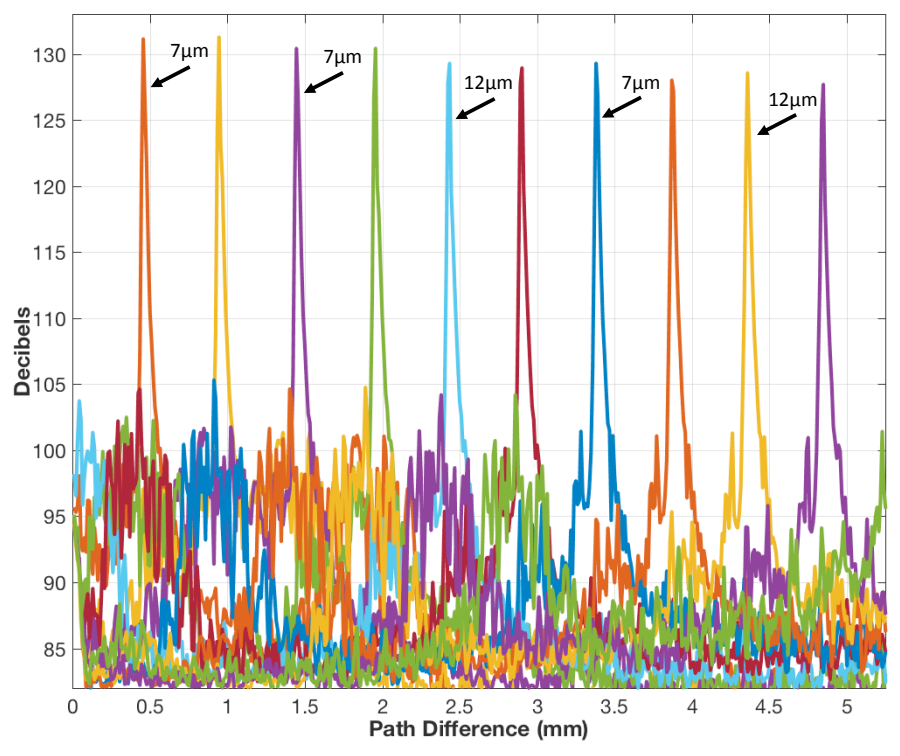

Figure 3.5: Point spread function (PSF) of the system with delayed MZI interferometer resampling clock to match round trip time of OCT light and $2^{\text {nd }}$ order software dispersion compensation. All axial resolution measurements are full-width, half-Max (FWHM). (Reprinted with permission of the Optical Society of America)

image (shown in figure 3.7). The leakage pulses cause the detector to saturate and hence no useful m-mode data can be acquired during this time. To solve this problem a time-multiplexed scheme was devised so that the fiber laser would only pulse during the portion of the OCT A-line cycle where no actual signal was being acquired.

The new timing scheme, shown in figure 3.8, was based around the A-line trigger pulse that is generated by the MEMS swept-source. The TTL-voltage signal, usually used to trigger the digitizer to begin execution of the A-line acquisition protocol, is split off and sent to the trigger input of an arbitrary waveform generator (Tektronix AFG3022C). The waveform generator is programmed to output $2 \mu \mathrm{s}$ pulses, $8.9 \mu \mathrm{s}$ after the rising edge of the A-line trigger. This signal is then fed to the laser diode driver (IXYS Colorado PCX-7420) that is responsible for driving the fiber laser's pump diode. Given that the fiber laser used in this system is gain-switched [104], pulsing of the laser occurs only during this time, and not during OCT acquisition. Figure 3.9 show the result of m-mode acquisition during percussion drilling into a piece of wood. Figure 3.10 shows the same for a piece of porcine scapula bone. Both images show total elimination of the vertical striping 


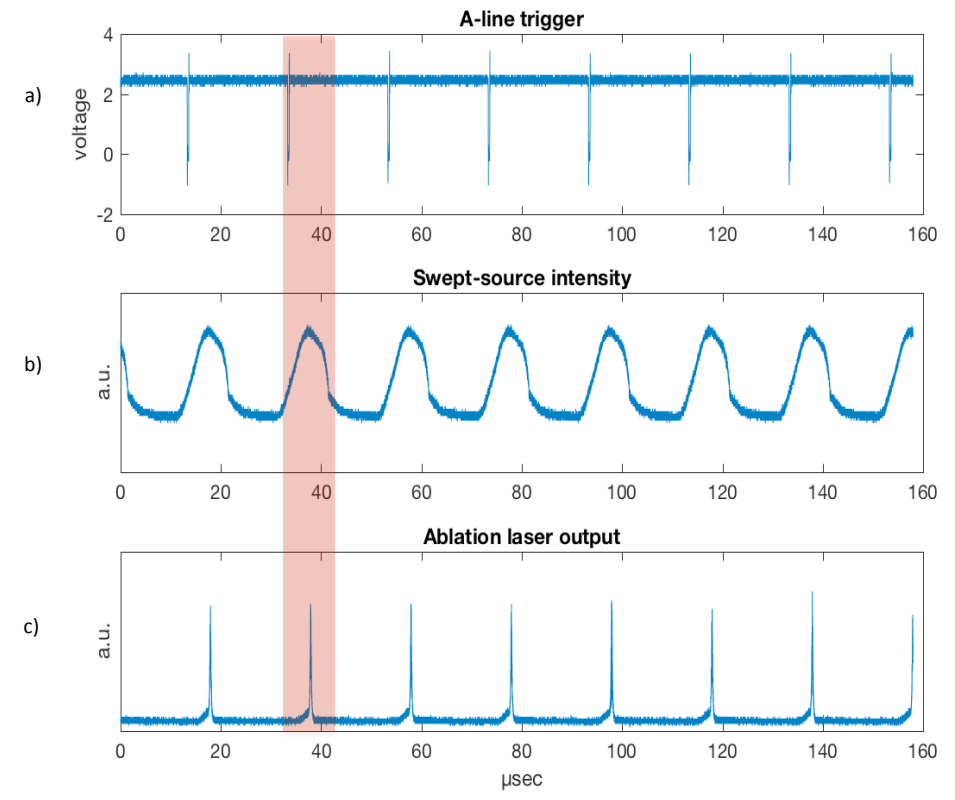

Figure 3.6: Timing relation between OCT data acquisition (A-line rate $=50 \mathrm{kHz}$ ) and ablation laser pulses $(50 \mathrm{kHz})$ when no synchronization is implemented (i.e. free-running ablation laser pulsing). The red box highlights which the portion of the cycle where the A-line acquisition takes place. It can be seen from the figure that the ablation pulses (c) clearly impinge on the OCT A-line (after the A-line trigger (a) and during the positive duty-cycle of the swept-source output (b). (Reprinted with permission of the Optical Society of America)

phenomenon.

\subsubsection{Ablation depth control implementation}

Feedback from the m-mode A-line acquisition was used for depth control in this work. The scheme that was implemented is shown in figure 3.11. The in-house developed software (based on [107, 108] was programmed to execute peak-detection, since it was assumed that the strongest peak of the Fourier spectrum would correspond to the reflection of where the surface of the specimen impinges the optical axis. For every incoming A-line, the position of the peak is detected on the Fourier axis and compared to a target position (defined by the user). A microcontroller is connected to the computer via USB serial communication. The microcontroller has a single digital output pin connected to a high-speed AND logic gate; the other input to the AND gate is connected to the output of the waveform generator (mentioned in the previous section) who's TTL output drives the laser diode driver. The output of the AND gate is connected to the driver's trigger input. The microcontroller's digital pin is by default set to logic 1, fulfilling the AND condition everytime a 


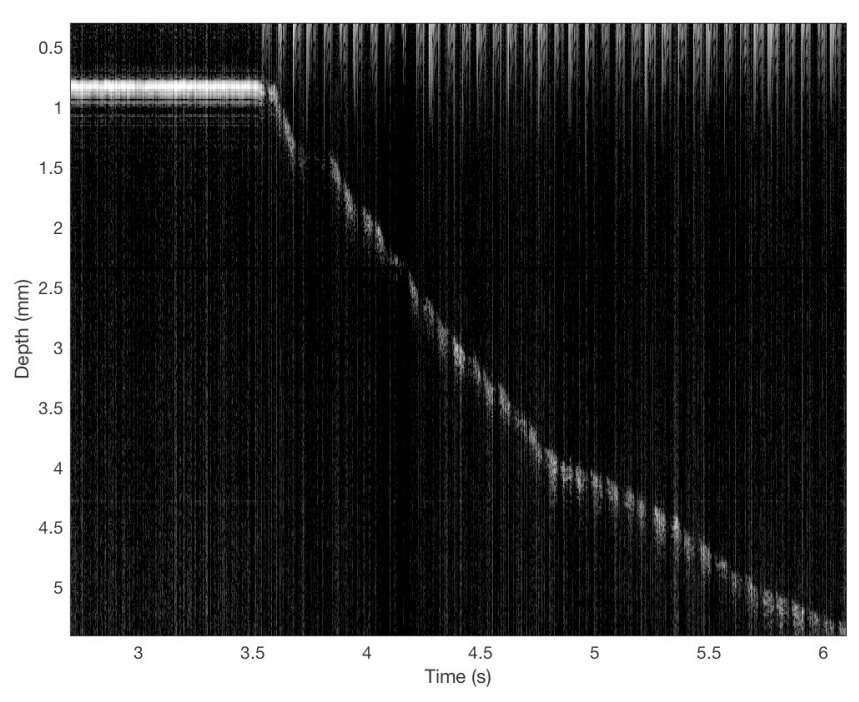

Figure 3.7: Depth progression of ablation front during ablation of wood (M-mode). Ablation laser induced artifacts are due to reflected energy through the fiber combiner saturating the balanced detectors. (Reprinted with permission of the Ontical Societv of America)

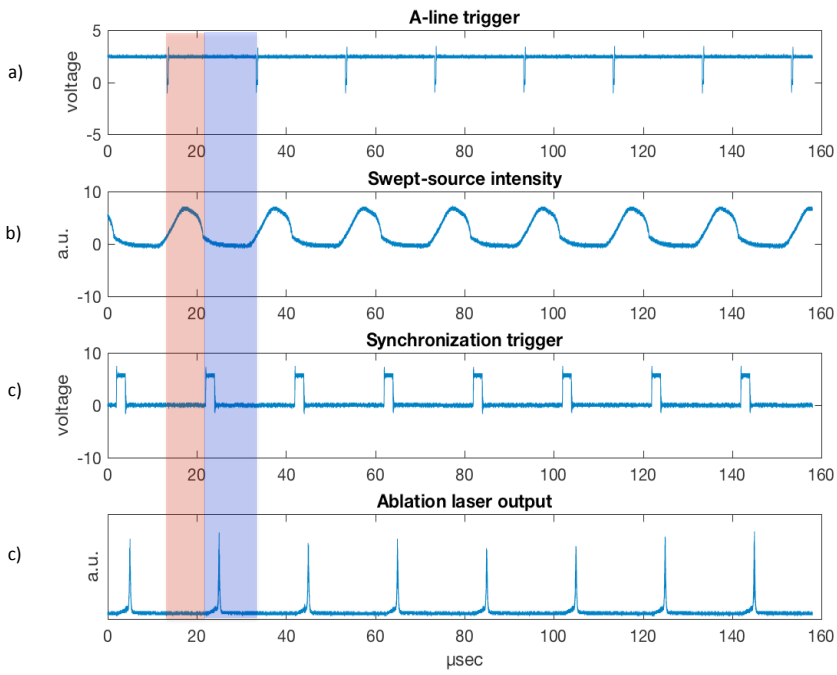

Figure 3.8: Timing relation between OCT data acquisition and ablation laser pulses with A-line triggered asymmetric pulse triggering timing scheme implemented. Red and blue boxes highlight portions of the cycle where A-line acquisition occurs and does not occur, respectively. Notice ablation (d) pulses occur only during the latter half of the cycle whilst no A-line acquisition is occuring (i.e. when swept-source output (b) is low). This is due to the fixed timing implemented between laser trigger pulse (c) and the A-line trigger (a). (Reprinted with permission of the Optical Society of America) 


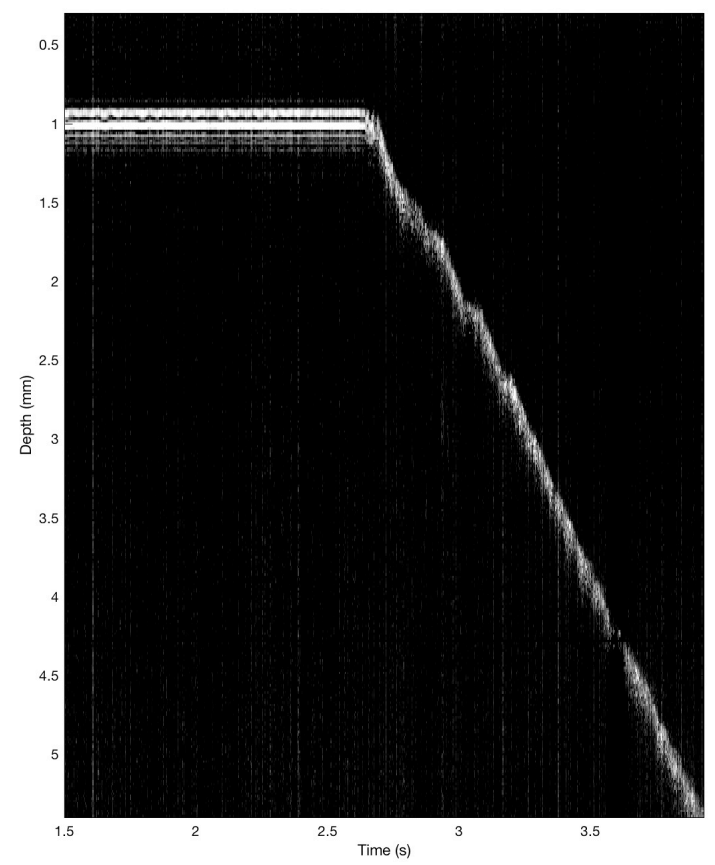

Figure 3.9: Depth progression of ablation front during ablation of wood (M-mode) using asymmetric pulse timing scheme. Ablation laser induced artifacts are no longer seen. (Reprinted with permission of the Optical Society of America) 


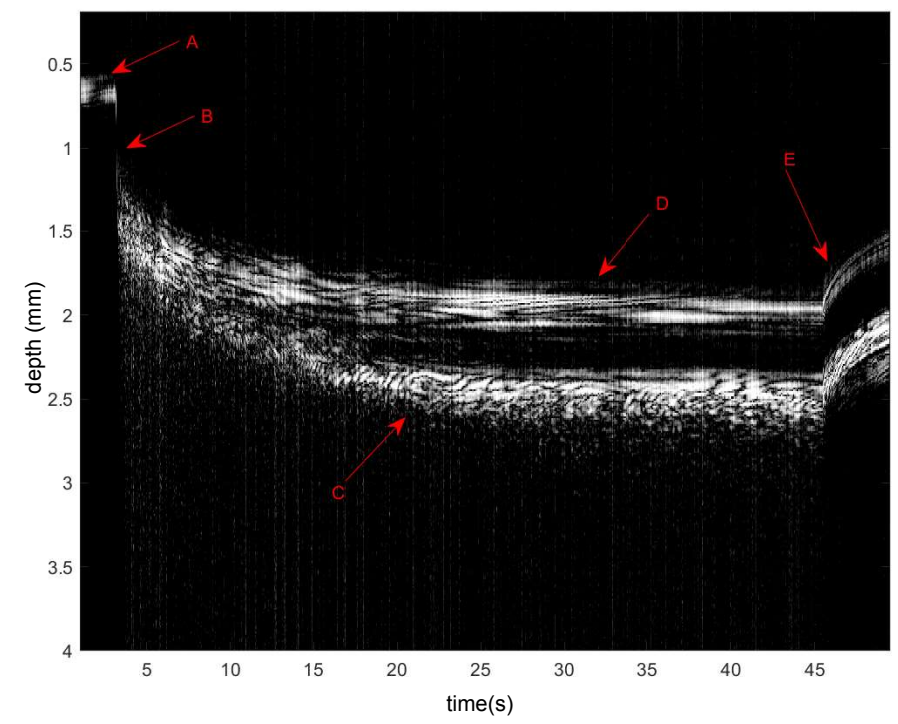

Figure 3.10: Depth progression of ablation front during ablative irradiation of fresh porcine scapula (Mmode) using the asymmetric pulse timing scheme described above. A: heating of distal cortical bone surface; $\mathrm{B}$ : penetration through cortical layer and center trabecular layer; $\mathrm{C}$ : heating of proximal cortical bone layer; D: hole side wall reflection; E: laser shut off, subsequent tissue relaxation/contraction. (Reprinted with permission of the Optical Society of America)

pulse is received from the waveform generator. Once the computer has detected that the peak has reached the target depth, a packet is sent to the microcontroller, commanding it to flip the digital pin to logic 0 , breaking the AND condition and stoping the ablation laser.

\subsection{Experiments and Results}

\section{Phantom drilling}

Phantoms made of medium density fiber board (MDF) were used to demonstrate the ability of the system, due to it's material uniformity and it's desirable response to thermal ablation. 12 holes were percussion drilled into the MDF phantom at 5 target depths $(0.5 \mathrm{~mm}, 1.0 \mathrm{~mm}, 1.5 \mathrm{~mm}, 2.0 \mathrm{~mm}$, $2.5 \mathrm{~mm})$, resulting in 60 holes in total. Axial depth targeting results are shown in figure 3.12 and summarized in table 3.2. The mean depths were measured using b-mode OCT scans taken using a separate, calibrated, OCT system. 


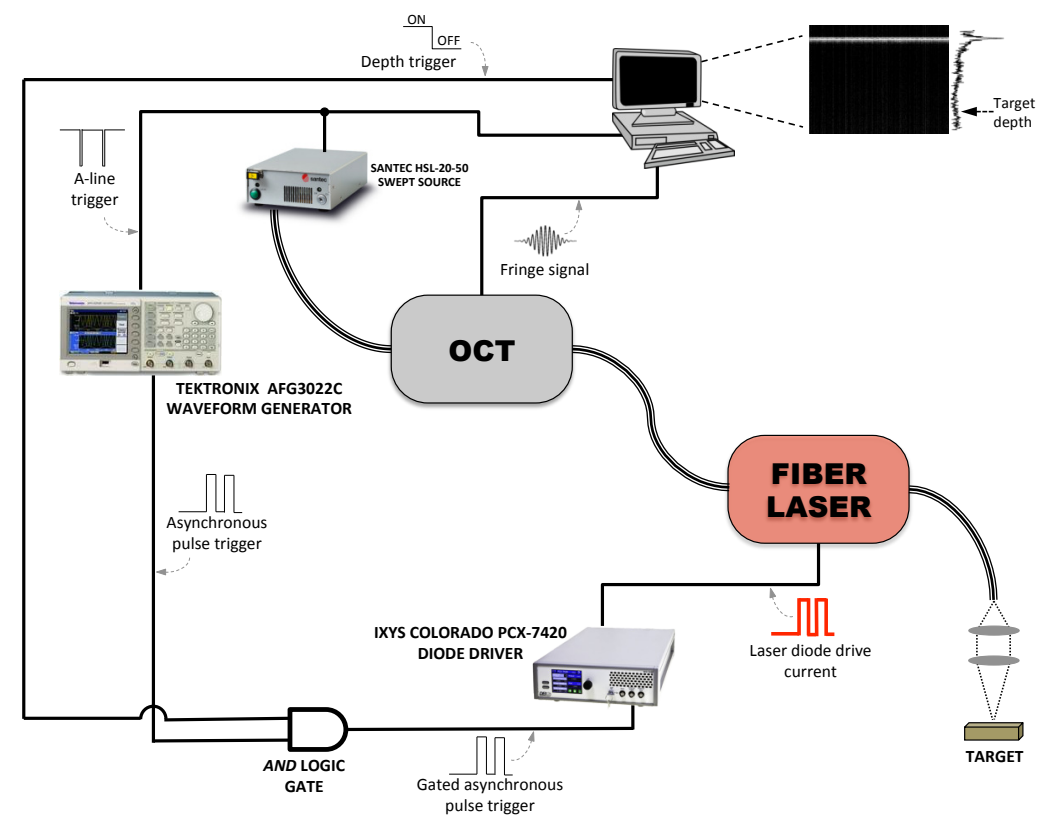

Figure 3.11: Diagram of system with integrated depth control. Detection of A-line peak at a target depth triggers a drive-signal shutoff to the ablation laser's pump diode driver. (Reprinted with permission of the Optical Society of America)

Table 3.2: Results summarizing drilling depth accuracy experiments on MDF phantom.

\begin{tabular}{|c|c|c|c|}
\hline Target Depth & Measured Depth & Mean error & Standard Deviation \\
\hline $0.5 \mathrm{~mm}$ & $0.52 \mathrm{~mm}$ & $0.020 \mathrm{~mm}$ & $0.042 \mathrm{~mm}$ \\
\hline $1.0 \mathrm{~mm}$ & $1.01 \mathrm{~mm}$ & $0.010 \mathrm{~mm}$ & $0.050 \mathrm{~mm}$ \\
\hline $1.5 \mathrm{~mm}$ & $1.47 \mathrm{~mm}$ & $-0.030 \mathrm{~mm}$ & $0.065 \mathrm{~mm}$ \\
\hline $2.0 \mathrm{~mm}$ & $1.957 \mathrm{~mm}$ & $-0.043 \mathrm{~mm}$ & $0.051 \mathrm{~mm}$ \\
\hline $2.5 \mathrm{~mm}$ & $2.42 \mathrm{~mm}$ & $-0.080 \mathrm{~mm}$ & $0.061 \mathrm{~mm}$ \\
\hline
\end{tabular}



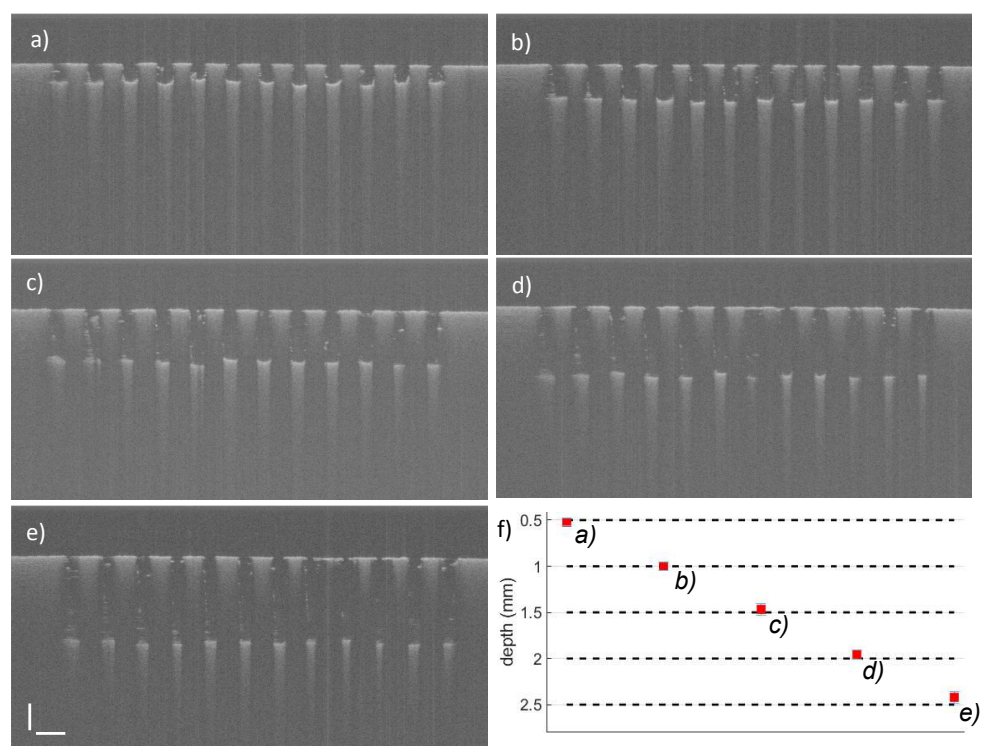

Figure 3.12: Ablation depth control trials on MDF phantom. Images a) to e) show OCT scans of the trials starting from $0.5 \mathrm{~mm}$ target depth to $2.5 \mathrm{~mm}$, incrementing by $0.5 \mathrm{~mm}$ respectively. Scale bars represent $0.5 \mathrm{~mm}$. Results are summarized in $\mathrm{f}$ ). Red squares represent mean measured depths with standard deviation of each panel figure, indicated by the each corresponding label. (Reprinted with permission of the Optical Society of America)

Table 3.3: Results summarizing drilling depth accuracy experiments porcine scapula specimens.

\begin{tabular}{|c|c|c|c|}
\hline Target Depth & Measured Depth & Mean error & Standard Deviation \\
\hline $0.3 \mathrm{~mm}$ & $0.306 \mathrm{~mm}$ & $0.060 \mathrm{~mm}$ & $0.071 \mathrm{~mm}$ \\
\hline $0.5 \mathrm{~mm}$ & $.0479 \mathrm{~mm}$ & $-0.021 \mathrm{~mm}$ & $0.054 \mathrm{~mm}$ \\
\hline
\end{tabular}

\section{bone drilling}

20 holes at two target depths, $0.3 \mathrm{~mm}$ and $0.5 \mathrm{~mm}$, were drilled into the cortical bone of porcine scapula specimens. Table 3.3 summarizes the measured depth of the bone drilling. The mean depths were measured using b-mode OCT scans taken using a separate, calibrated, in-house OCT system.

\subsection{Discussion and Conclusion}

In this chapter, the novel integration of a swept-source OCT system and an ablation fiber laser is demonstrated, as well as the ability to execute depth-controlled ablation with sub-millimeter accuracy using this system. This all-fiber topology can be applied to medical and non-medical ablation 
applications. It was also demonstrated in this chapter that bending losses of $1310 \mathrm{~nm}$ light used for OCT is significant in through the passive Nufren SM-GDF-10/125 fiber. A phenomenon that showed OPD-dependant peak broadening was described, and a successful solution was proposed. A second solution is proposed in the addendum.

The lack of dichroic mirror in this system proves to be advantageous with respect to space constraints. The system can be made extremely compact, and more importantly the output stage can be realized with a single collimating lens and a single focusing lens; this makes it ideal for mounting onto a robotic end-effector, as is a salient theme in throughout this dissertation. It is predicted that the body of the laser structure can be realized in a package the size of a text book, since the two largest components are the Santec HSL-20-50 swept-source (194x135x53mm) and the RealLight M976 $\pm 3-110-F 105 / 22-D 1$ pump diode $(85 \times 58 \times 20 \mathrm{~mm})$. By virtue of the system being all-fiber, coaxial beam coupling is inherent and the need for free-space beam alignment is eliminated. As well, using this structure allows for large laser power disparities between the OCT system and the ablation laser. Power scalability is also achievable using this structure since deeper or an increased number of TFBGs can be used, increasing the ablation laser power rejection ratio but still remaining virtually transparent to the OCT swept-source light.

This structure does have some potential limtations. First, the highly dispersive nature of the fiber does create problems for the OCT imaging. We have demonstrated a solution to this using software-based dispersion compensation, however that does cause and increase in computational overhead. Another problem is the significant attenuation of the of OCT light due to the large mode area fiber used to construct the ablation laser. This does significantly does degrade OCT SNR. Although we have demonstrated the successful use of an optical amplifier to increase the overall swept-source power through the entire system, the backscatter travelling from the sample and back through the same fiber is subject to the same high attenuation. However, since the surface of the ablation kerf is most prominent feature, depth control is still easily realized. One strategy that was adopted in order to mitigate bending losses was through the use of an inexpensive armoured sheath to encase the output pigtail. This limited the bend radius to greater than approximately $60 \mathrm{~mm}$, 
greatly reducing the bending attenuation effect. Armoured optical cables such as this are already adopted in many industrial and medical applications that require any sort of critical optical fiber outside to be protected from mechanical and even chemical damage.

We attribute the realized error being larger than the resolution system due to control loop, which slower than the ablation laser pulse rate. Increasing loop speed could potential achieve greater targeting accuracy, however the reported targeting accuracy in this chapter surpasses the suitability for bone drilling. As well, faster computation electronics or faster communication protocols between the computer and microcontroller could significantly speed up control loop frequency, but could potentially increase system cost and complexity as well. The obvious limitation of the peak detection algorithm could include false depth targeting due to spurious reflections being interpreted as peaks, as well as decreased backscattering due to carbonized tissue becoming the dominant absorber during drilling progression. Using this novel structure, top-down ablation control was successfully demonstrated. This system has the potential to visualize sub-surface interfaces well before penetration by the ablation laser.

\subsection{ADDENDUM 1: Alternate method for sample arm cou- pling of fiber laser}

Figure 3.13 shows an alternate method for coupling the fiber laser to the sample arm of the OCT system. This method uses free-space bulk optics instead of inline fiber gratings. Two collimating lenses are placed in-line between the sample arm and fiber laser. Between the two collimators are two Thorlabs FEL1150 edge-pass filters (cut-off wavelength at 1150nm). The attenuation of the $1064 \mathrm{~nm}$ light going into the OCT system was measured to be $63 \mathrm{~dB}$. Although this method was not a realization of the full fiber design, it was demonstrated with success in [9]. Results from this structure is shown in 3.14 


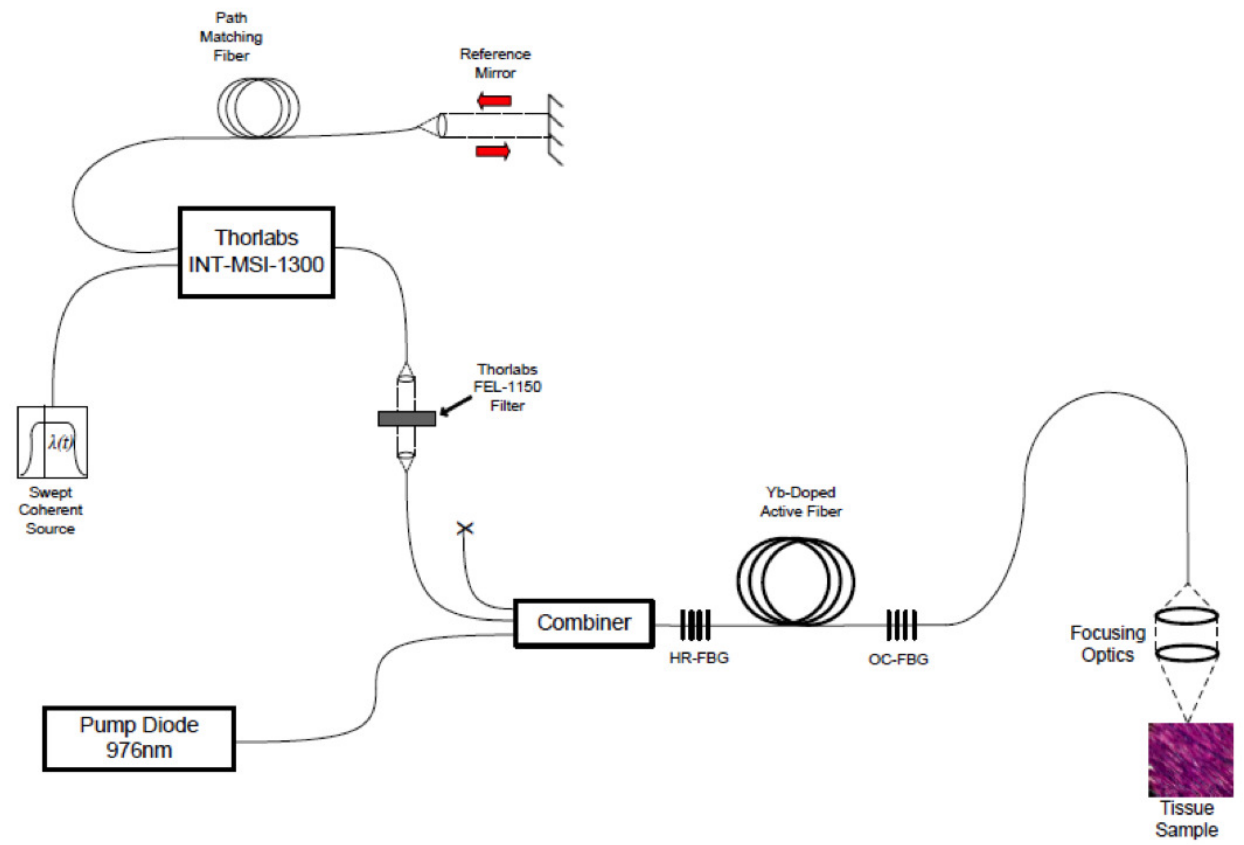

Figure 3.13: Schematic showing alternate method for coupling fiber laser into the sample arm of the OCT system. Note that this method eliminates the all-fiber design of the sample arm optics. 


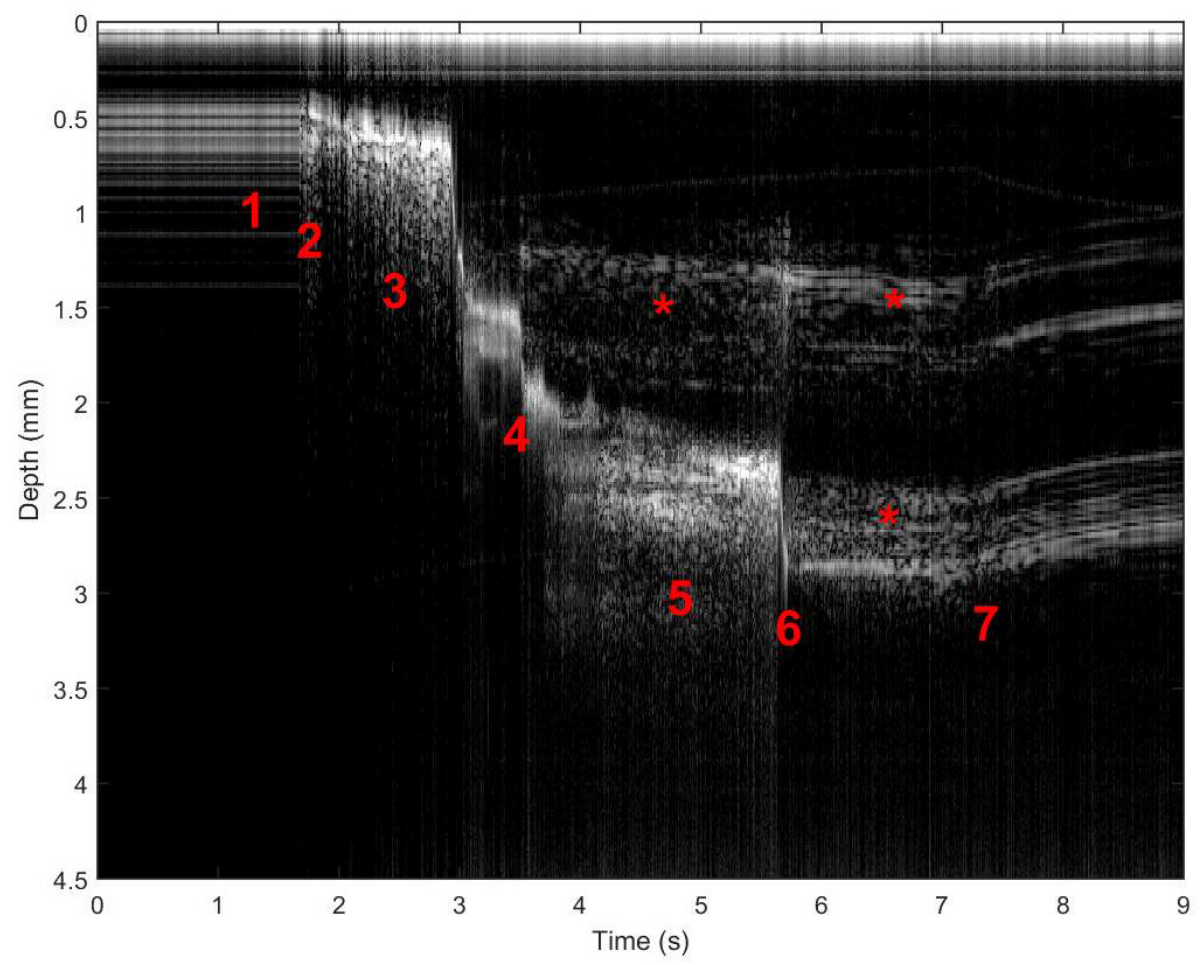

Figure 3.14: Depth progression of ablation front during ablative irradiation of fresh lamb scapula (M-mode) using alternate coupling structure and software dispersion compensation. 1: ablation laser off; 2: ablation laser switched on; 3: ablation of superior cortical bone layer; 4: ablation of inner trabecular bone layer; 5 ablation of inferior cortical bone layer; 6: breach of inferior bone-air interface; 7: laser switched off ; *: side walls of ablated hole. 


\subsection{ADDENDUM 2: Alternate method for k-clock delay}

As described in this chapter, the k-clock had to be delayed in order to match the delay caused by the OCT light making a round-trip time through the fiber. This was solved optically using a delayed Mach-Zehnder sampling clock; the delay was induced by adding a proportional length of SMF-28 fiber to the clock input. However, an electrical solution was also experimented with which used an amplifier and proportional length of coaxial conductor in order to achieve a comparable delay of the original k-clock electrical signal generated by the swept-source unit. The signal was first amplified using a Mini Circuits ZHL-6A RF amplifier (500MHz bandwidth), then sent through roughly $45 \mathrm{~m}$ of coaxial cable. This is shown in figure 3.15 .

The signal delay of the cable and amplifier resulted in a delayed k-clock signal that was practically useful. Figure 3.16 a) shows the PSF after implementation of the electrical k-clock delay but before software dispersion compensation. All peaks are seen to be uniformly broadened, which is expected due to dispersion mismatch caused by the sample and reference arms being constructed of different fibers. Figure 3.16 b) shows the PSF after using same software dispersion compensation method used with the optical method. The figures show that this method is practically viable. However, this method showed the peaks of the PSFs rising as a function of optical path difference. This was thought to have been caused by slightly imperfect matching of the required k-delay. In the end, the optical method was chosen in the end due to easier tunability of the delay.

Although the optical method previously detailed yielded an overall better PSF, this electri-

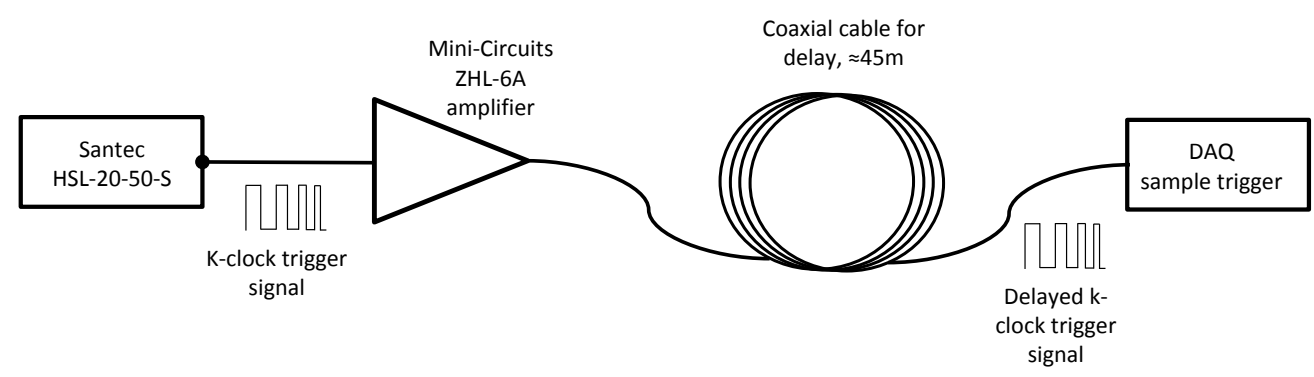

Figure 3.15: Schematic showing electrical based scheme for k-clock sample trigger delay. 

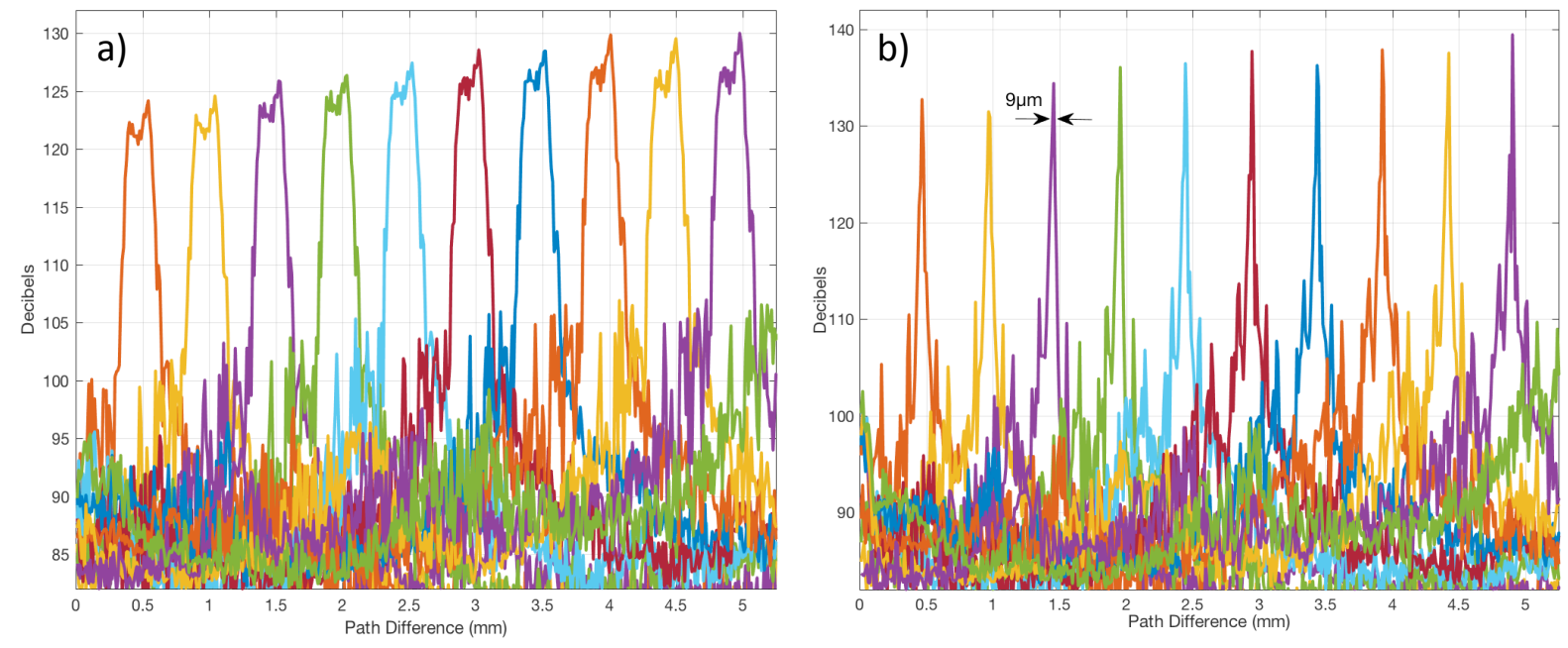

Figure 3.16: a) Point spread function (PSF) of the system with k-clock delay to match round trip time of OCT light. Peak broadening is now approximately uniform; irregular peak shapes are due to high dispersion introduced by the inline fiber laser. b) Point spread function (PSF) of the system with k-clock delay to match round trip time of OCT light and $2^{\text {nd }}$ order software dispersion compensation. Both figures show rising amplitude as a function of optical path difference, which is not to be expected.

cal method does offer a practical solution at a significantly reduced cost. The cost of the highbandwidth electrical amplifier and long coaxial cable is estimated to be roughly \$430 CAD at the time this dissertation was written $(\approx \$ 150 \mathrm{CAD}$ and $\$ 280 \mathrm{CAD}$ for coaxial cable and amplifier, respectively). To demonstrate the cost disparity, a comparable integrated Mach-Zehnder interferometer clock such as the Thorlabs INT-MZI-1300 has a current listed price of \$2556.38 CAD (\$1944.66 USD); the electrical solution is roughly one-sixth the cost of the comparable optical method. This cost disparity could potentially be greater not only because the cost of the extra delay-inducing fiber must be cosnidered, but also because the INT-MZI-1300 outputs a $200 \mathrm{MHz}$ k-clock signal; the optical method described in this chapter necessitated a custom-built MZI clock with a roughly $300 \mathrm{MHz}$ bandwidth. The cost of the custom clock is estimated to be much larger than the integrated solution. 


\section{Chapter 4}

\section{Robotic Trajectory Generation via Structured Light Penscription}

The work presented in this chapter resulted in the following journal publication [109]:

Jivraj, J., Deorajh, R., Lai, P., Chen, C., Nguyen, N., Ramjist, J., and Yang, V. X. (2019). Robotic laser osteotomy through penscriptive structured light visual servoing. International journal of computer assisted radiology and surgery, 14(5), pp. 809-818.

\subsection{Introduction}

Robotic systems potentially offer higher precision and repeatability during surgery, however usability remains a significant limiting factor. The current paradigm for trajectory planning in robotic osteotomy is done on a computer system, usually before the procedure, using a pre-surgical CT scan $[110,111]$. This approach is non-intuitive and limited in terms of flexibility (ie. depending on how the exposure turns out and whether other factors that only become obvious during surgery might necessitate a change in the original plan). Also, complex shapes and geometries that are easily realized by hand may be harder to realize with a purely robotic system, even though modern robotic manipulators have the potential to be more dexterous and could have a larger range of motion compared to humans. Robots potentially have the ability to surpass humans in surgical ability [112], but it is important that trajectory generation methods not be as complex as the capabilities of the robot itself. 
In this chapter, a very intuitive interaction method for the surgeon to guide the robot where to perform the osteotomy is presented. This method makes use of standard, high-contrast, sterile surgical pens or pencils (readily available in any operating room) to demarcate cutting trajectories. By having the robot follow a hand-drawn line that is directly penscribed on the exposed bone surface, the experience becomes very similar to the current situation of a senior surgeon teaching a resident and directly marking where the cut is to be made. This method also allows for osteotomy of complex trajectories that a human can draw easily by hand, but with the additional precision, repeatability and potential speed (feed rate) a robotic system can provide.

\subsubsection{Related Work}

As mentioned above, current schemes proposed to robotic laser osteotomies involve trajectory generation on screen, using pre-surgical imaging, and not directly on the patient tissue/anatomy $[112,113,114,115,116]$. Similar work to the method described in this chapter, where a robotic system follows a high-contrast line on the workpiece, has been carried out in the real of visionguided robotic welding. In this area, a robotic manipulator (typically 6-DOF) with a welding torch end-effector is visually guided along a seam between two plates of metal. This has very obvious parallels to robotic laser osteotomy because the welding payload, just like an optical payload, has to be guided along a defined trajectory at a specific orientation with respect to the surface, and at a specific stand-off distance to the workpiece.

Schemes using structured light have been used to guide robots along welds. A light stripe and laser scanning system has been used by Xu et al. to identify the weld seams between metal plates [117]. The was demonstrated using a method that directed a laser line to perpendicularly intersect with the seam to be welded $[117,118,119,120,121]$. A a plano-convex cylindrical lens was used to expand a collimated red laser beam into a straight line. A camera captured an image of the workpieces to be welded together, along with the laser line. The width of the same was calculated by analyzing the laser line's turning points on the image. A passive CCD (without a laser line) was 
used by Ye et al. and to detect the center of a target seam during pulse metal active gas (pulseMAG) welding through the center of the weld pool in real-time during welding [122]. Given that the welding process produces more than enough light to saturate the CCD, highly attenuating filters optical filters were placed in the optical path. To isolate the edges of the same in order to find the center, common image processing techniques: mean filtering, Sobel edge detection, image dilation, image erosion, area filtering and edge searching via Hough transform were used, in that order. The error was reported to be $\pm 1 \mathrm{~mm}$ from the seam. A similar process was extended to gas metal arc welding (GMAW) by the Xu et al. [123]. Using improved image processing techniques, the error was reported to be reduced to $\pm 0.3 \mathrm{~mm}$.

\subsection{System Description}

\subsubsection{Vision system}

A custom, in-house built surgical navigation prototype system previously used in [124] was used in this chapter. The system included of a digital micromirror device (DMD) projector-based structured light imaging system integrated with an infrared tool tracker (Vicra, Northern Digital Incorporated), built into a surgical light head. This was mounted onto a multi-DOF surgical light arm which allowed positiioning above a surgical field in the same way as is done in a standard operating room (OR) setting with a standard, ceiling-mounted surgical light.

\subsubsection{Robotic hardware}

The robot used in this chapter was the A KUKA LBR14 IIWA, a compact, 7-DOF manipulator arm that is designed to work in close collaboration with humans, such as in the OR. The robot was mounted onto a in-house built prototype cart designed to be used patient-side during surgery. An end-effector with focusing optics and a planar IR fiducial tracking frame was mounted onto the most distal DOF of robot. Inverse kinematics were not required to be calculated since the robot software and interface is designed to move the payload to any point in the robot's workspace, defined by Cartesian coordinates; joint-space angles are determined internally within the robot 
controller. Orientation of the payload is defined by Euler angles, allowing for full attitude control in a simple, command-based way.

\subsubsection{System integration}

Figure 4.1 shows the complete system diagram. The coordinate space of the IR tracking camera within the surgical navigation system is taken as the reference frame for the entire system. That means that the point cloud gathered by the DMD-based structured light (SL) system is referenced to the IR tracking camera. Position and orientation data of the payload based on the fiducal tracking frame is also within the same coordinate space. Since the fiducial frame is rigidly connected to the robot, a homogenous Cartesian transform was used to transform the Cartesian coordinates of the SL surface into the robot's working space (i.e. with respect to the robot's origin) [125]. Custom, in-house designed software was developed to perform this transformation in real-time at a rate of $20 \mathrm{~Hz}$ (this was limited by the framer rate of the IR tracking camera). This software allowed for trajectories to be defined directly using cartesian data from the SL surface and transformed into direct position and orientation set-points for the robot to follow, no matter what orientation the robot payload was initially in with respect to the navigation system (as long as the fiducal tracking frame remained unoccluded). These targets are sent to the robot point-by-point via ethernet communication.

\section{Optical Payload}

A Thorlabs F810APC-1064 collimator and A Thorlabs AC254-150-C achromatic doublet focusing lens were fixed in a lens tube were mounted onto the robot payload. Through this optical set up, laser ablation and OCT imaging were done for system characterization. An in-house built fiber laser with the following parameters was used in this experiment: $\lambda_{c}=1064 \mathrm{~nm}, P_{a v g}=4 W$, $L_{\text {focal }}=14.7 \mathrm{~cm}, D_{\text {spot }}=45 \mu \mathrm{m}$. The OCT imaging system was a MEMS-based swept source system with a $50 \mathrm{kHz}$ a-line rate and approximately $9 \mu \mathrm{m}$ axial resolution. Both ablation laser and OCT imaging were done using the robot mounted lens setup via an APC-terminated armoured fiber 


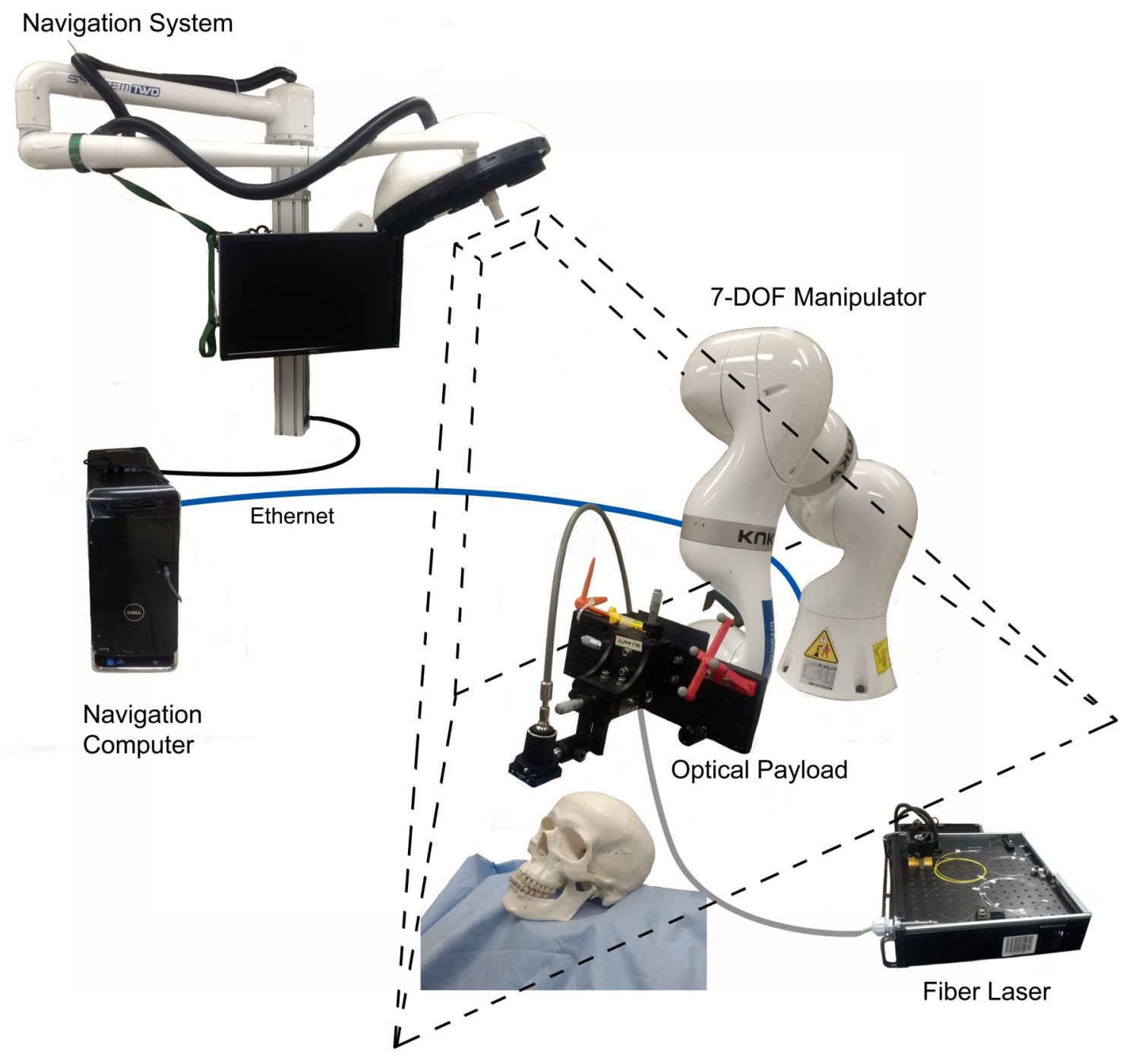

Figure 4.1: Diagram of complete system. Tracking and structured light optics are housed in the surgical light head. Payload of robot guides the objective lens of the fiber laser ablation system over the target. Cartesian data is sent to the robot via ethernet by the navigation computer. 
pigtail connected to the collimator.

\subsubsection{Workflow}

The workflow for the system is proposed as follows:

1. The surgeon performs the initial soft tissue exposure above target area of the craniotomy. The scalp is pulled back, revealing only the boney surface of the skull.

2. The surgeon uses a sterile surgical pencil or pen (color being in high-contrast to the bone) and draws an outline of the desired craniotomy by hand.

Note: the previous two steps typically exist in a standard craniotomy protocol. The third step would be the drilling by hand; the hope is that the next four steps would take less time than this.

3. The navigation system is positioned above the exposure and an SL surface is taken. On screen, the surgeon or an assistant picks two points: the first point being where the surgeon would like the cut to begin, the second being the approximate radius and/or center point of the trajectory. Note that this only has to be approximate.

4. At this point, the software, using the algorithm described in the following section, segments the trajectory and generates all necessary cartesian position and pose information for the robot to follow.

5. The robot is wheeled in place with it's payload in vicinity of the surgical cite and being full view of the navigation system.

6. Upon the surgeon's command, the robot ablates the bone along the drawn line, autonomously. 


\subsection{Algorithm}

\subsubsection{Acquisition and Isolation}

Once the user draws a trajectory on the exposed bone surface, an SL point cloud $\mathrm{S}_{1}$ of the surgical site is acquired. $\mathbf{S}_{1}$ consists of an $n$ by 7 matrix $\left[p_{n_{x}} p_{n_{y}} p_{n_{z}} N_{n_{x}} N_{n_{y}} N_{n_{z}} c_{n}\right.$ ], where $n$ is the number of surface points acquired, $P_{n}=\left[p_{n_{x}} p_{n_{y}} p_{n_{z}}\right]$ are the cartesian coordinates of each point, $N_{n_{x}}, N_{n_{y}}, N_{n_{z}}$ are vector normals to the surface at each point, and $c_{n}$ is a relative contrast value (0-255) of each point. The point cloud is displayed on the screen, and the only input to the GUI by the used is required at this point. Two points are selected on the digitized 3D surface; the first point $u_{1}$ marks where the surgeon would like to start the cut, and the second point $u_{2}$ marks the approximate center of the trajectory; This is shown in figure $4.2 \mathrm{~b}$ ). The euclidean distance, $d_{1}$ is then calculated.

A simple distance threshold operation is performed where all the points in $\mathbf{S}_{\mathbf{1}}$ that fall within $d_{1}$, centred at $u_{2}$ are preserved in set $\mathbf{S}_{\mathbf{d}}$ :

$$
P_{n} \in S_{d} \Leftrightarrow d_{n} \leq\left(d_{1}+d_{f}\right)
$$

where $d_{n}$ is the euclidean distance between $u_{2}$ and point $P_{n}$ and $d_{f}$ is a factor that extends the radius of the sphere slightly further to ensure the entire trajectory is included; typically $d_{f}<<d_{1}$. $\mathbf{S}_{\mathbf{d}}$ therefore consists of $\left[p_{m_{x}} p_{m_{y}} p_{m_{z}}\right.$, where $m$ is the number of points in $\mathbf{S}_{\mathbf{d}}$ and $m<<n$.

\subsubsection{Image space mapping and detection}

The point cloud $\mathbf{S}_{\mathbf{d}}$ was flattened to 2-dimensional XY-space since the depth coordinate information alone does not represent any unique points in the space; unique points are purely determined by what was visible in the XY-plane during acquisition. This way, simple image processing techniques can be used to isolate the trajectory information and the corresponding depth information $\mathrm{Z}$ can be introduced back without any loss of data or perspective. To flatten the point cloud into an 


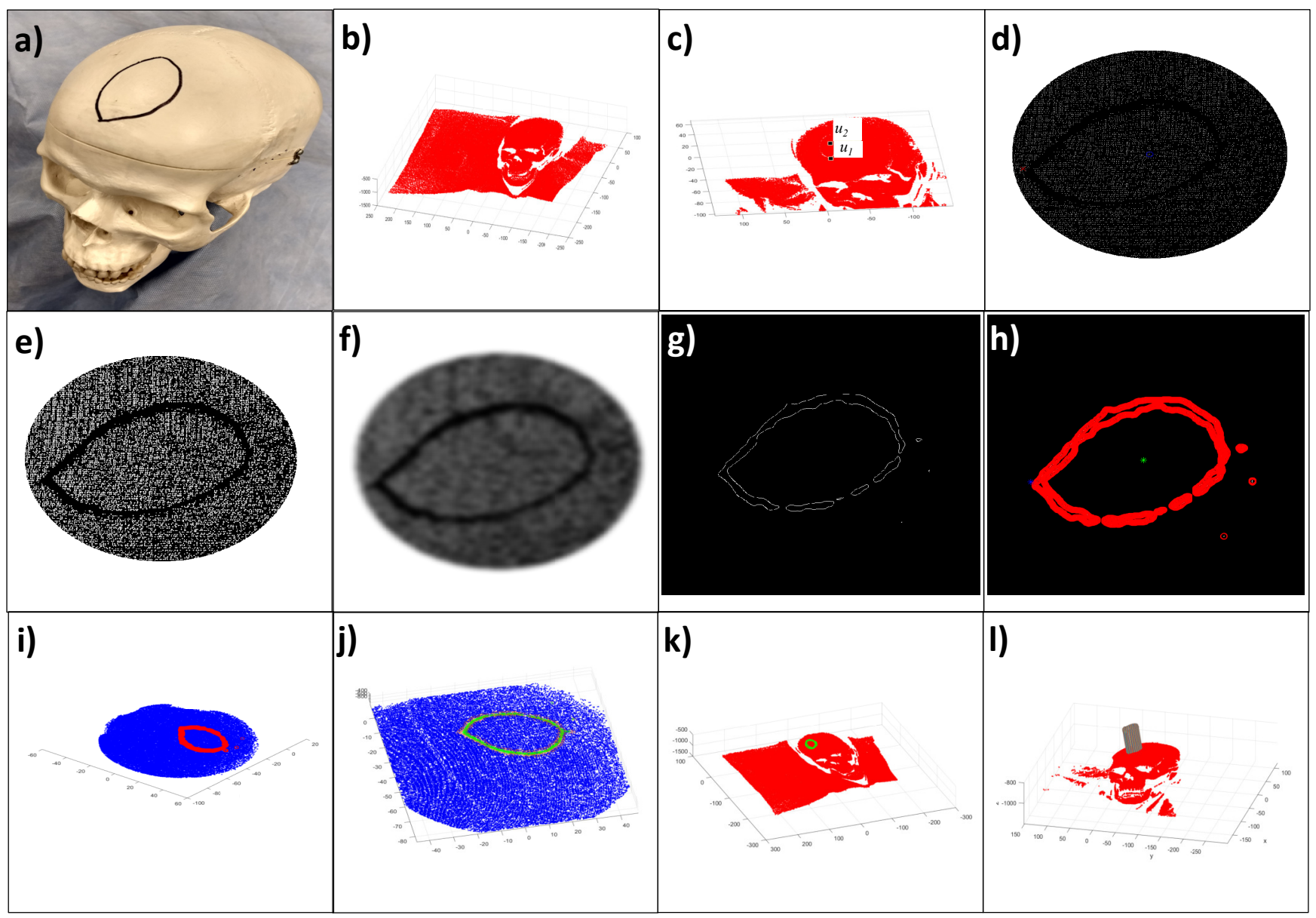

Figure 4.2: Steps of the penscription detection algorithm. a) Phantom with penscription. b) View of the entire SL surface. c) Points picked by user (start point and approximate center). d) Mapping to flattened image space. e) Image dilation of mapped image. f) Gaussian blur of mapped image. g) Sobel edge detection filter. h) Nearest neighbour growth algorithm to detect residual points that represent penscription. i) Mapping back to image space. j) Skeletonization of trajectory via averaging. k) Trajectory after multi-order least squares polynomial fit. 1) Normals for end-effector orientation generated using principal component analysis. 
image representing the $\mathrm{XY}$ plane, each $\left[p_{m_{x}} p_{m_{y}}\right]$ coordinate pair is assigned to a pixel in the image space. However, by the nature of the acquisition system, the points are not captured with uniform spacing. This non-uniformity arises because the acquisition system captures the point-cloud data with a certain distribution; that is, even though the system has a quoted resolution of $0.5 \mathrm{~mm}$, not all points within the SL surface are spaced $0.5 \mathrm{~mm}$ apart in all three dimensions. Without uniformity, common image processing techniques will have difficulty in simple tasks such as edge detection.. Therefore, an image $\mathbf{I}_{\mathbf{1}}$ is first established where each pixel represents a uniform spacing that is smaller than the resolution of the system (in this case, $0.1 \mathrm{~mm}$ was chosen to be sufficiently small), ensuring every point in $\left[p_{m_{x}} p_{m_{y}}\right]$ will have a unique corresponding pixel to be mapped to in image space. The size of $\mathbf{I}_{1}$ is $i \times j$ pixels, where

$$
\begin{aligned}
& i=\left\lceil\frac{\max \left(p_{m_{x}}\right)-\min \left(p_{m_{x}}\right)}{0.1}\right\rceil \\
& \text { and } \\
& j=\left\lceil\frac{\max \left(p_{m_{y}}\right)-\min \left(p_{m_{y}}\right)}{0.1}\right\rceil
\end{aligned}
$$

The points $\mathbf{S}_{\mathbf{d}}$ can be negative or positive floating point numbers, but in image space the pixels are assigned only positive value integers. The following relations map every point in $\mathbf{S}_{\mathbf{d}}$ to a corresponding pixel in image space $\mathbf{I}_{1}$, ensuring a one-to-one positive integer mapping of all positive and negative values in $S_{d}$ with the most negative values starting at 1 in image space:

$$
\begin{gathered}
\text { XofPixel }=\frac{\left\|p_{m_{x}}-\min \left(p_{m_{x}}\right)+0.1\right\|}{0.1} \\
\text { YofPixel }=\frac{\left\|p_{m_{y}}-\min \left(p_{m_{y}}\right)+0.1\right\|}{0.1}
\end{gathered}
$$




\section{Filtering and edge isolation}

The contrast value of every pixel in $I_{1}$ is initialized to 0 (black). Each point in $S_{d}$, defined by coordinates $\left[p_{m_{x}} p_{m_{y}}\right.$ ], is mapped to $\mathbf{I}_{\mathbf{1}}$ by setting the contrast of each corresponding pixel to a value of 255 (White), generating a binary image, shown in figure $4.2 \mathrm{c}$ ). The resulting image is very sparse, with fairly large discontinuities (black space) between the white pixels corresponding to surface points. In order to use further image processing techniques, the discontinuities are filled in using a simple morphological dilation operator, found in [126]. Rhomboid dilation of between 5 -15 pixel edge length was chosen through experimentation. The result of the image dilation is shown in figure $4.2 \mathrm{~d}$ ). Before the dilation, the pixels that represented the outline of the cutting path are sparse and non-contiguous. After dilation, the trajectory becomes contiguous and made isolation more effective.

Since the outlined cut path tends to be a large feature in the image, a gaussian blur of (0.2-0.5 standard deviation) is performed to low-pass filter the image in order to remove smaller features and high frequency artifacts caused by the dilation. Next, a Sobel filter is used to isolate edges on the low-pass filtered image [127]. Figure $4.2 \mathrm{f}$ ) shows the edge well isolated from any surrounding noise or spurious features after the Sobel filter. The resulting image after processing will be referred to as $\mathbf{I}_{2}$.

\section{Iterative Nearest Neighbour (INN) growth algorithm}

Once the edge is well isolated, an iterative growth algorithm based on the Iterative Nearest Neighbour method is performed to capture all the points in the trajectory, without capturing surrounding noise which may still be present and could be incorrectly interpreted as part of the cut path. The previous steps are imperative because the INN method relies on good isolation of the lowfrequency feature. The algorithm first designates $u_{1}$ as a pixel of interest (POI) and finds neighbouring pixels in a small surrounding neighbourhood area that have non-zero pixel contrast values

(the image is no longer binary because of gaussian blur and Sobel filter previously performed) and chooses the closest one. This pixel is then stored in a new set, discarded from the original set, and 
becomes the new POI. The process is continued for a maximum number of iterations $v$. Formally:

1. The image $\mathbf{I}_{2}$, consists of an $i$ by $j$ grid of pixels, each with an associated contrast value $c$ (between 0 and 255). The goal is to isolate pixel set $\Phi$, which contains $l$ unique pixels that approximate the outline of the cut path; $l<<k$ where $k=i j$ and $\Phi \subset \mathbf{I}_{\mathbf{2}}$.

2. for $n=1, P O I_{n}=u_{1}$.

3. for $n=2 \ldots v, P O I_{n}=\phi_{n-1}$

4. The pixel distance between each $P O I$ and all pixels in $\mathbf{I}_{2}$ where $c \neq 0$ is calculated and closest neighbouring point $\phi \in \Phi$ is found. $\phi$ is "removed" from the $\mathbf{I}_{\mathbf{2}}$ by setting $c=0$. When $\phi_{n}=\phi_{n-1}$, the algorithm is terminated, leaving $l$ unique points. Typically, $v$ is chosen such that $v>l$.

\section{Mapping back to cartesian space}

Given that the cartesian coordinates $\left[p_{m_{x}} p_{m_{y}}\right]$ for all $\mathbf{S}_{\mathbf{d}}$ were preserved as pixel coordinates $[i$ $j$ ] using equations 4.4 and 4.5 , a reversal of the process is done in order to find the corresponding points in the point cloud represented by each pixel.

$$
\begin{aligned}
& p_{m_{x}}^{\prime}=\min \left(p_{m_{x}}\right)+0.1 \phi_{i}-0.1 \\
& p_{m_{y}}^{\prime}=\min \left(p_{m_{y}}\right)+0.1 \phi_{j}-0.1
\end{aligned}
$$

At this point, the corresponding z-coordinates $p_{m_{z}}^{\prime}$ are re-introduced to give a complete 3dimensional approximate isolation of the cut-path, denoted as $\mathbf{S}_{\mathrm{o}}$ (where $\mathbf{S}_{\mathrm{o}} \subset \mathbf{S}_{\mathbf{d}}$ ). This is shown 
in figure $4.2 \mathrm{~h}$ ).

\section{Averaging and Skeletonization}

As mentioned before, the isolation of the cut-path is in actuality isolating the edges of a void that is shaped as the cut-path, due to the high-contrast black marking on the surface of the bone. This presents the problem of filling in points in that void where the data does not exist. To begin to solve this, iterative spatial averaging is performed on the points of $\mathbf{S}_{\mathbf{d}}$ that lie in proximity of $\mathbf{S}_{\mathbf{o}}$. This is done by taking the mean of all the points in $\mathbf{S}_{\mathbf{d}}$ that fall within a predefined radius of each point in $\mathbf{S}_{\mathbf{o}}$. The reasoning is that the collective points in the radius $r_{d}$ will closely approximate the actual surface of where the penscribed void lies becausebecause most bones, with the exception of irregular bones, do not exhibit sharp discontinuities (i.e. spikes or pits) within a distance that would be typical of the line thickness. The new resultant point is saved in set $S_{\mathbf{p}}$ and this process is continued for all points in $\mathbf{S}_{\mathbf{o}}$. Formally,

$$
S_{p}=\left[\frac{1}{b} \sum \rho_{p_{x}} \frac{1}{b} \sum \rho_{p_{y}} \frac{1}{b} \sum \rho_{p_{z}}\right]
$$

where $\left[\rho_{p_{x}} \rho_{p_{y}} \rho_{p_{z}}\right]$ are the coordinates of the points that fall within $r_{d}$ of each point in $\mathbf{S}_{\mathbf{o}}$. This process is repeated for all points in $\mathbf{S}_{\mathbf{o}}$. Figure $4.2 \mathrm{j}$ ) shows the outcome of this.

\subsubsection{Natural Direction Ordering and polynomial fit Re-ordering to follow natural path direction}

The trajectory thus far is populated in a matrix that is randomly ordered. If any robotic system is fed this data in it's current sequence as waypoints, the motion of the robot will be unpredictable. The cartesian points therefore need to be ordered in a "natural" order, meaning in a way one would sequentially and intuitively follow the trajectory - i.e. along the cut-path, and in a consistent direction relative to that cut-path. A seemingly simple problem, this becomes complicated given that the initial surface is randomly oriented with respect to the world reference frame and no single 
dimension or natural vector along the surface lies perfectly along any of the three cardinal world axes.

First, $u_{1}$ is selected as the first point $t_{1}$ in the sorted trajectory $\mathbf{T}$. All the points in the randomly sorted trajectory are arranged from smallest to largest euclidean distance away from it. The closest point $n n_{1}$ to $t_{1}$ is then isolated, and the direction vector between the two, $\mathbf{n}_{1}$, is calculated. $n n_{1}$ is chosen as $t_{2}$ (the next point in $\mathbf{T}$ ) and $u_{1}$ is discarded from the original, non-ordered set. After this, the process for arranging the randomly sorted points from smallest to greatest euclidean distance is repeated with respect to $t_{2}$ rather than $t_{1}$. This time, the closest $t w o$ points $n n_{1}$ and $n n_{2}$ are isolated and the direction vectors between each of them and $t_{2}, \mathbf{n}_{\mathbf{2}_{1}}$ and $\mathbf{n}_{\mathbf{2}_{2}}$, are calculated. The angles between these two direction vectors and the previous direction vector $\mathbf{n}_{\mathbf{1}}$ is calculated. The point corresponding to the smallest of these two angles is taken as the next point $t_{3}$, discarded from the original set, and this entire process is repeated until there remains no more points in the original, un-ordered set. The resulting set $\mathbf{T}$ has all the points ordered by distance and angular proximity. This results in a trajectory that approximately follows the natural order of the original cut-path with some high frequency jitter.

\section{Polynomial fit and jitter filtering}

Once the trajectory has been re-ordered to follow the cut-path, a multi-order polynomial fit is performed on the data to approximate the cut-path into a smooth curve and remove high frequency translational jitter. The polynomial fit is done using a least mean square error method [128]. Each axis is fit separately and the final trajectory is is combined into the matrix $[X Y Z]$, where:

$$
\begin{aligned}
& X(m)=c_{x_{1}} m^{n}+c_{x_{2}} m^{n-1}+\ldots+c_{x_{n}} m+c_{x_{n}+1} \\
& Y(m)=c_{y_{1}} m^{n}+c_{y_{2}} m^{n-1}+\ldots+c_{y_{n}} m+c_{y_{n}+1}
\end{aligned}
$$




$$
Z(m)=c_{z_{1}} m^{n}+c_{z_{2}} m^{n-1}+\ldots+c_{z_{n}} m+c_{z_{n}+1}
$$

where

$$
m=1 \ldots \text { length }(\mathbf{T})
$$

and $c_{x}, c_{y}$ and $c_{z}$ are the polynomial constants of each individual axis.

The order $n$ of the fit is increased relative to the complexity of the cut-path, but it was found upon experimentation that a $20^{t h}$ order fit worked well in most situations without any spurious oscillations. The result of the polynomial fit is shown in figure $4.2 \mathrm{k}$ ).

\subsubsection{Surface normal generation}

Once the cartesian coordinates representing the trajectory have been calculated, a vector that is normal to the surface and pointing distally with respect to the body needs to be calculated to give the desired orientation of the payload optical axis. A commonly used method of statistical analysis known as principal component analysis (PCA) [129] is used in this method to find the the vectors that originate on the surface of the scan and point away from the center of the body, referred to as the distal normal vectors. PCA uses the variances of a multivariate dataset to find linear orthogonal vectors, or components, within the data, which could be interpreted as useful relationships upon analysis and interpretation. Here, we take advantage of this method because we assume that small clusters of points along the surface point cloud (points in the trajectory and within proximity) approximates a plane. This plane can be defined by the first two principal components that represent the two largest directions of variance within the cluster; the third component will naturally give a vector perpendicular to the first two and approximately normal to the cluster.

Point $t_{i}$ in $\mathbf{T}$ is selected as the center, and a cluster of points within a fixed radius (typically on the order of 5-10mm) are selected. For each cluster we effectively define a new set of three axes by weighting each of the components of $\mathbf{T}$ by a set of three weighting factors $\mathbf{W}$. Formally: 


$$
w_{k}=\arg \max \left(\frac{\mathbf{w}^{\mathbf{T}} \widehat{\mathbf{T}}_{\mathbf{k}}^{\mathbf{T}} \widehat{\mathbf{T}}_{\mathbf{k}} \mathbf{w}}{\mathbf{w}^{\mathbf{T}} \mathbf{w}}\right)
$$

where $k=1 \ldots 3$ for each of the three principal components based on the three principal axes $(\mathrm{x}, \mathrm{y}, \mathrm{z}) . \widehat{T}$ is the new data set with previous principal component removed:

$$
\widehat{\mathbf{T}}_{\mathbf{k}}=\mathbf{T}-\sum_{n=1}^{k-1} \mathbf{T} \mathbf{w}_{\mathbf{n}} \mathbf{w}_{\mathbf{n}}^{\mathbf{T}}
$$

The rows of the $3 \times 3$ matrix $\mathrm{w}$ defines the unit vectors of the three principal components. The first two define the cluster surface approximation, and the third results in the distal surface normal. This process is repeated for each point in $\mathrm{T}$ such that every point will have it's own associated distal surface normal vector. The result of this is shown in figure 4.21 ).

\subsection{Experiments}

\subsubsection{Deviation in target plane}

10 straight lines, approximately $5 \mathrm{~cm}$ in length were drawn onto wooden blocks and were used as cutting targets. Wood was used as a substrate because of its ability to be consistently and clearly marked by the laser. A fiber laser $\left(\lambda_{c}=1064 \mathrm{~nm}, P_{\text {avg }}=4 W, L_{\text {focal }}=14.7 \mathrm{~cm}, D_{\text {spot }}=45 \mu \mathrm{m}\right)$ was coupled to the focusing optics of the robot payload in order to trace the followed cut-path by directly ablating the substrate during path execution. A fast moving air stream over the cutting area, provided by a pressurized air hose, was used to evacuate smoke and eliminate flame generation. Image processing techniques were used to calculate the accuracy of the cut after each trial. A picture of the wooden block after the cut was taken and the penscribed line and the cut line were isolated. The center-lines of both were approximated using a multi-order polynomial fit and the mean euclidean distance at each point on the center-line of the cut from the center-line of the penscribed line was calculated. Figure 4.3 show this distance error along the progression of the cut for one of the trials. The mean distance error is $0.46 \mathrm{~mm}$ for the straight line cut trials and a 


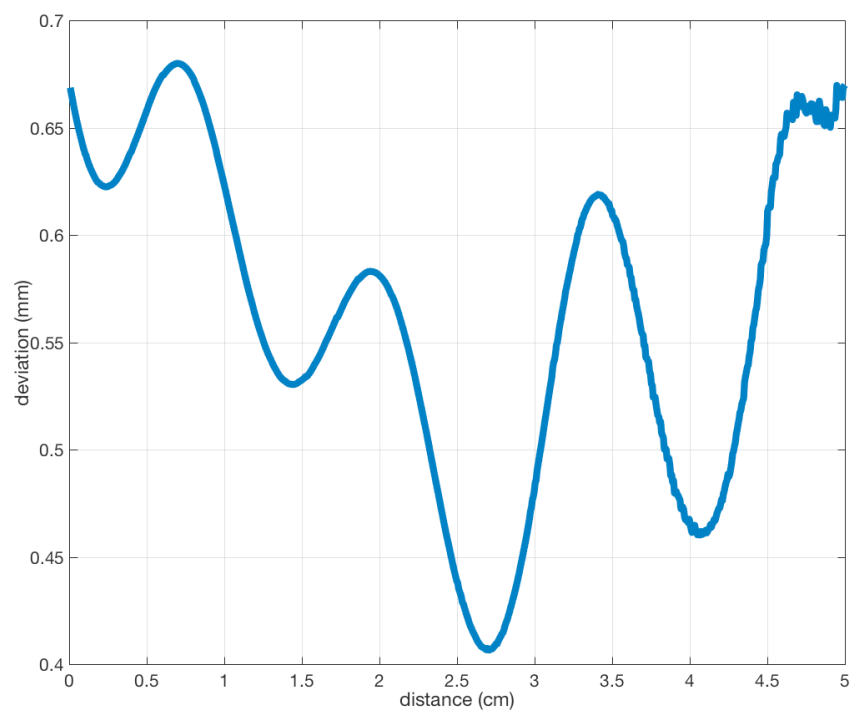

Figure 4.3: Error between penscription and ablated kerf (centerline-to-centerline) for a single straight line cutting trial.

standard deviation of $0.36 \mathrm{~mm}$. As seen in figure 4.4, as the block was moved in the YZ-plane of the tracking volume, there was a slight increase in cartesian error. It is known by [130, 131] that NDI Polaris tracking camera does have a non-homogeneous distribution in its tracking volume, and this is likely the main source for variations observed in the mean error and standard deviation through $n=10$ trials.

\subsubsection{Deviation along optical axis}

Having the laser beam impinge the surface of the cut right at the beam waist is important for cutting efficiency and volumetric confinement of the ablation. Therefore, the deviation along the optical axis was measured to quantify focal shift with respect to the surface of the target. Just as before, 10 straight lines approximately $5 \mathrm{~cm}$ in length were drawn onto a piece of medium density fiber board. The 10 targets were placed in the workspace of the robot and the imaging system. Optical coherence tomography (OCT) was used to measure optical axis deviation by coupling the beam into the robot-mounted optical payload. As the robot moved the optical payload along 


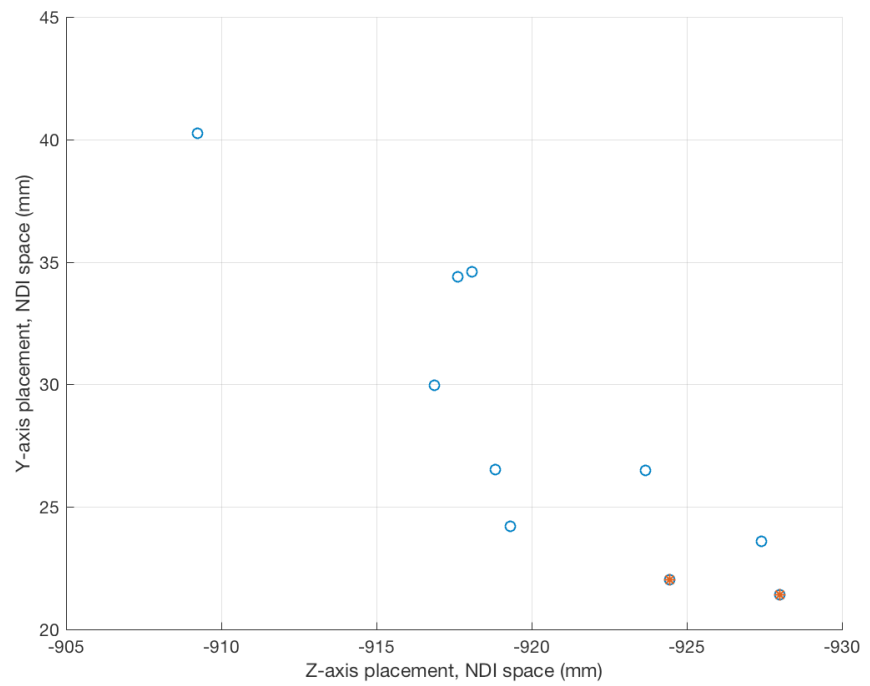

Figure 4.4: Placement of target center in YZ-plane of the NDI Polaris tracking volume. The two red points represent the trials with the highest error found (approximately double the mean). This suggests that a sweet-spot of where the target should be placed in the tracking volume.

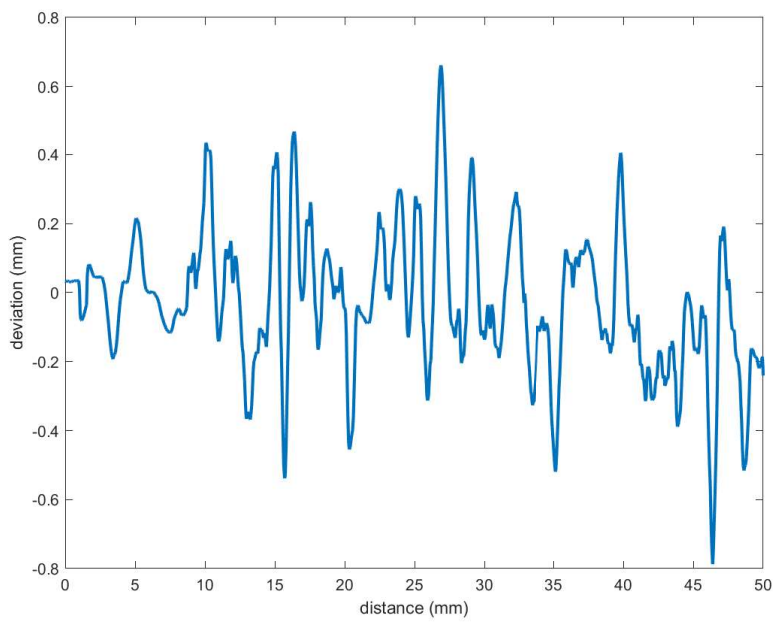

Figure 4.5: Deviations along optical axis for a single straight line cutting trial, measured using m-mode OCT. 
the penscribed line on the target, m-modem images were captured at an a-line rate of $50 \mathrm{kHz}$; movement in the optical axis was seen as surface shift between a-line scans. The results from a single trial are shown in figure 4.5. The mean of all the trials was found to be $0.36 \mathrm{~mm}$ with a standard deviation of $0.16 \mathrm{~mm}$.

\subsubsection{Reproduction of surgical osteotomy trajectory}

To demonstrate the viability of this system for use in osteotomy, a reproduction of a real surgical osteotomy performed at Sunnybrook Health Sciences Centre (Toronto, CA) was performed on a pig scapula. The individual steps for the algorithm execution are shown in 4.6. The trajectory, $4.7 \mathrm{~b}$ ) (outlined in red for visual clarity) was hand sketched onto the bare bone of a fresh porcine scapula, shown in 4.6 a) and figure $4.7 \mathrm{c}$ ). The result of the cut is shown in $4.7 \mathrm{~d}$ ). The mean deviation from the penscribed path was found to be approximately $0.53 \mathrm{~mm}$.

\subsection{Discussion and Conclusion}

In this chapter a novel method for planning of osteotomies in surgical interventions is presented; primarily craniotomies in neurosurgery. Existing robotic surgical systems lack intuitive interaction and planning schemes, and therefore potentially hinder adoption. Our method presents a highly intuitive planning method that takes advantage of the current common practice of drawing targets onto a boney surface for osteotomy with a sterile pencil or marker. The only training required of a surgeon or OR staff would be in executing placement of the two points on the SL surface (starting point and approximate center) using a CAD-based GUI. The sub-millimeter accuracy achieved in the work described in this chaper is comparable to studies involving robotic bone drilling [132, 133, 134]. According to [135], we navigation systems are deemed acceptable with a target registration error between $1.0-1.5 \mathrm{~mm}$; we have achieved an mean error well below this whilst using robotic drilling guided by a clinical navigation system. We believe that this technique 


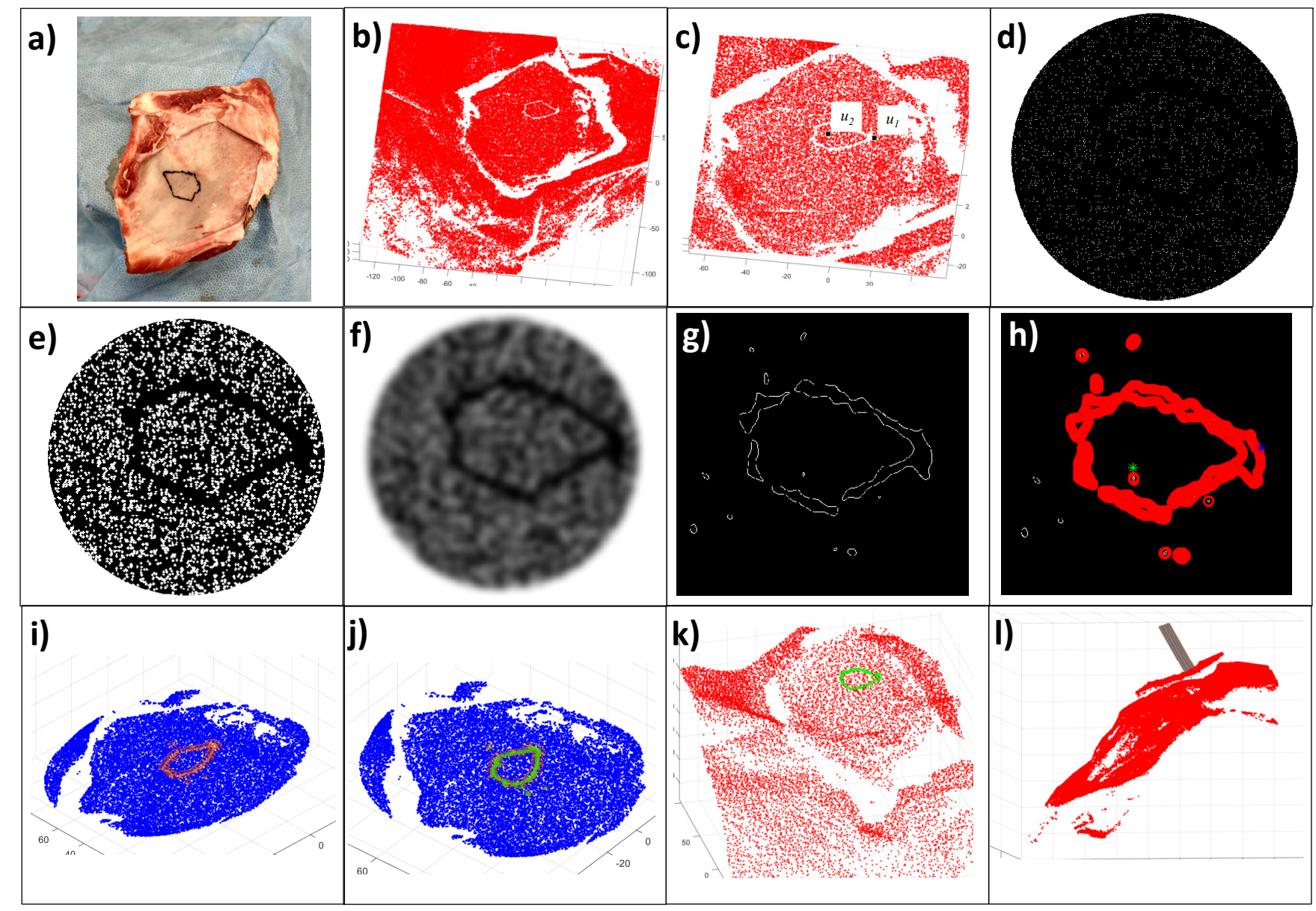

Figure 4.6: Algorithm steps for osteotomy experiment. a) Scapula with penscription. b) SL surface. c) User selected points. d) Mapping to flattened image space. e) Image dilation. f) Gaussian blurred. g) Sobel edge detection filter. h) Nearest neighbour growth algorithm. i) Mapping back to image space. j) Skeletonization via averaging. k) Post multi-order least squares polynomial fit. 1) Normal vectors generated using principal component analysis. 

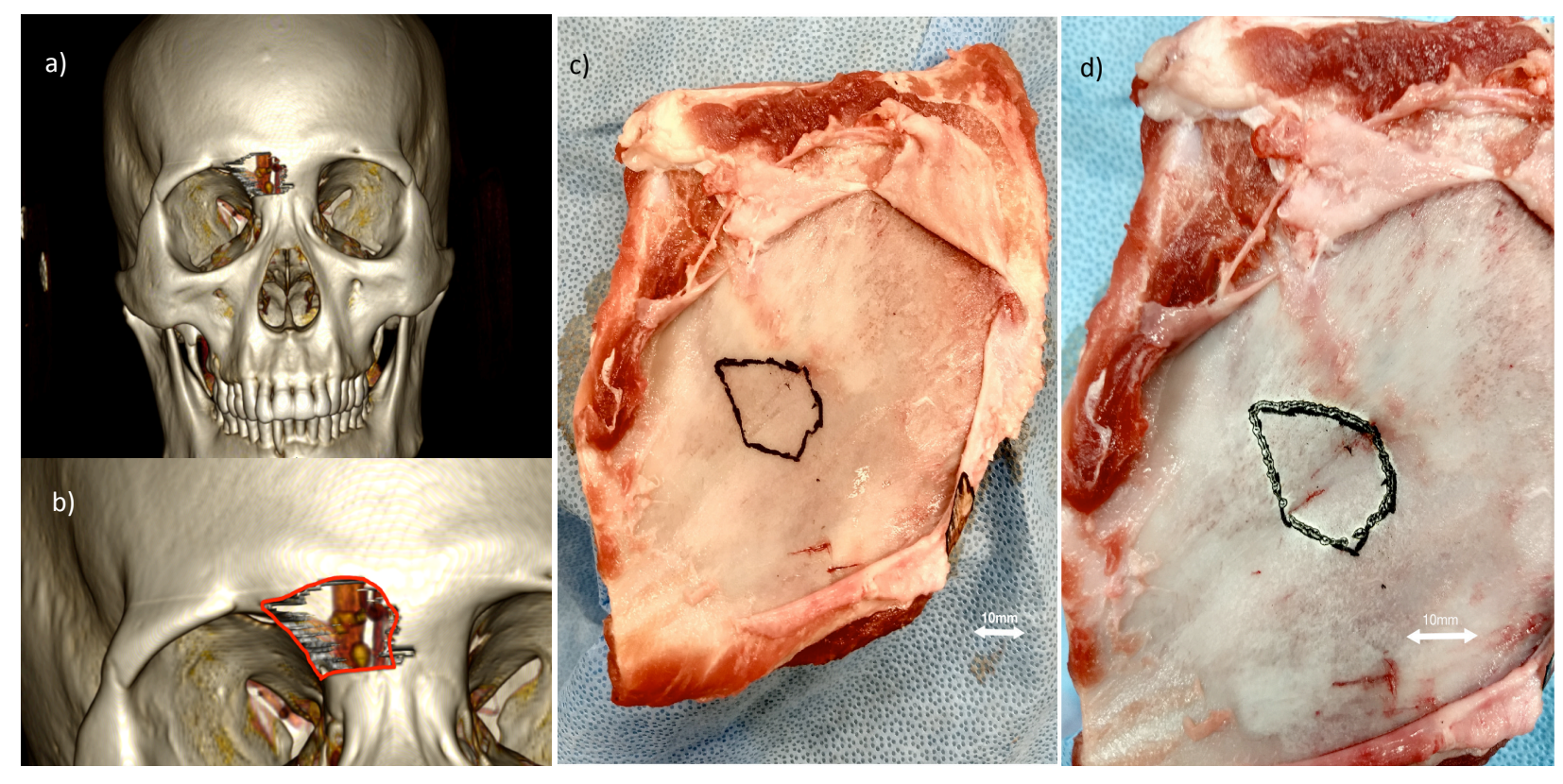

Figure 4.7: a) Patient scan of a frontal ethmoidal sinusectomy. b) Red outline showing trajectory. c) Trace of osteotomy outline onto porcine scapula. d) Ablated trajectory.

can allow for more advanced cuts that may be difficult to produce by hand. For example, our technique can allow for simple change in orientation of the laser beam with respect to the bone surface; this requires simply applying angular offsets to the normal vector that is determined using the PCA-based technique described in section 3.4. By manipulating beam orientation, it is possible to achieve complex edge profiles of the bone flap, such as chamfer, mitre, fillet, etc. As well, beam orientation manipulation can potentially allow for the robot to target the beam in scenarios of over-hang obstructions. This technique can be used with various lasers with different etch rates, since multiple passes over the same trajectory (for multi-pass full penetration) is simply achieved by biasing the values of the trajectory along the optical axis by a fixed amount with every pass. This would bring the beam focus further and further down inside the kerf with each pass. Our hope is that this planning methodology can be used in future robotic systems in order to make the speed of procedures quicker and make the use of robotic systems in the OR less intimidating and cumbersome. 


\section{Chapter 5}

\section{Bone Ablation Etch Rate Optimization Using Nitrogen as an Assist Gas and Graphite as a Topical Absorber}

The work presented in this chapter resulted in the following journal publication [136]:

Jivraj, Jamil, Chaoliang Chen, Dexter Barrows, Xijia Gu, and Victor XD Yang. "Optimization of laser osteotomy at $1064 \mathrm{~nm}$ using a graphite topical absorber and a nitrogen assist gas jet.” Biomedical Optics Express 10, no. 7 (2019): 3114-3123.

\subsection{Introduction}

In this chapter, etch rate optimization of laser bone ablation at $1064 \mathrm{~nm}$ using the laser system described in Chapter 3 is explored. This is done using a coaxial nitrogen assist gas jet and graphite as a topical chromophore. Nitrogen is commonly used OR gas, making it ideal as a potential optimizing agent. Graphite is chosen, aside from it's high absorption cross section, because in Chapter 4 the method of pencription for robotic trajectory generation was developed; the ability of a sterile surgical pencil to serve additionally as an ablation optimizer is an attractive prospect. 


\subsubsection{Topical chromophores}

The use of topical chromophores have been studied in laser skin resurfacing applications to increase thermal ablative damage for improved resurfacing results. Sumain et al. proposed the viability of carbon-based topical chromophores on skin with $532 \mathrm{~nm}$ laser irradiance for increased laser light absorption and subsequent heat transfer to dermal tissue [137]. The results showed that the coagulation depth was dependant on laser dose, and it was claimed that the use of the carbonbased chromophores were effective. However, no bare-skin irradiance baseline was established for comparison.

Indocyanine green (ICG) based topical dye has been used in select studies as a dermal topical absorber to increase thermal damage depth for skin resurfacing. Diven et al. showed that the use of ICG based dye on human cadaveric and guinea pig skin in conjunction with laser irradiation at $805 \mathrm{~nm}$ produced significant penetration of thermal damage compared to irradiation on bare skin. It was reported that with application of an ICG-based dye, thermal-induced coagulation was seen to be as high as $416 \mu \mathrm{m}$ immediately after ablation on live guinea pig specimens. It is important to note that an inverse relation between irradiation power and coagulation depth was seen when samples were immediately compared via histology just after ablation; $3 \mathrm{~W}$ of laser irradiation produced deeper damage penetration $(416 \mu \mathrm{m})$ than at $10 \mathrm{~W}(390 \mu \mathrm{m})$. A baseline of $0 \mu \mathrm{m}$ damage at $10 \mathrm{~W}$ was established when no dye was used. However, after 1 week and 1 month, depth of scarring was seen to positively correlate with irradiation power [138]. Mordon et al. experimented with the efficacy of ICG aqueous solution versus ICG emulsion (phosphatidylcholine and soybean oil) injections in laser photocoagulation of blood vessels in hamster skin flaps [139]. It was demonstrated that a 50 and 60 percent reduction in laser fluence at $805 \mathrm{~nm}$ was achieved using the ICG emulsion compared to the aqueous solution to achieve vessel coagulation and skin ablation, respectively. Roh et al. experimented with various fluence and exposure parameters of a 1064nm ND:YAG laser for treatment of enlarged facial pores. One group of subjects in the study was treated with a topical lotion of suspended carbon particles prior to ablation. This study proved the efficacy of $1064 \mathrm{~nm}$ ablation for pore size reduction, however no significant difference was seen with the application of the topical lotion. [140]. 
For osseous tissue, Kang et al. showed a three to six fold increase in ablation crater depth and volume by using a thin layer of distilled water on top of the target bone [141]. The thin layer of water was found to have decreased the damage threshold for the bone.

Ultrashort pulsed (USP) lasers have shown promise in laser osteotomy, since the peak-powers achieved using these types of lasers are easily able to induce non-thermal interaction (such as optical breakdown); because of this, little to no heat is generated at the ablation site. However, USP lasers do suffer some drawbacks. The very large field intensities achieved can potentially cause self-focusing due to spatial index variations caused by the Kerr effect [142]. This can be avoided by using high numerical aperture (NA) optics, but that would inherently lead to shorter stand-off (focusing) distances of the objective optics, which may potentially reduce or negate the general non-contact benefit of laser surgery. Given that the penetration depth of ultrashort pulses is extremely shallow (approximately $1 \mu \mathrm{m}$ due to highly efficient absorption by surface plasma), etch-rates of USP lasers tend to be comparatively slow. As well, USP lasers have much larger costs and system complexity compared to nanosecond pulsed fiber lasers.

For wavelength-multiplexed applications, high peak powers generated by USP lasers may provide challenges when optically coupling with coaxial monitoring beams, such as in applications using optical coherence tomography (OCT) based monitoring and control [89, 143]. High peak powers could lead to potentially damaging leakage into common optical paths.

Thermal damage to an ablation target is a concern that is explored in multiple studies, as excessive thermally-induced necrosis will prevent or delay healing [144, 145, 146, 147]. Ablation in the thermal regime is limited by excessive heat accumulation and diffusion into tissue. Therefore, reduced exposure time to the laser for a given target volume would be advantageous since it would limit the overall of heat deposition and diffusion into the surrounding tissue. An interesting study was done by Sotsuka et al., where bone ablation at 1070nm was compared with saw based osteotomy [148]. It was found that there no significant differences in healing times between the two. However, it was concluded that the laser ablation was a combination of thermal and plasma mediated ablation, therefore less charring was seen around the osteotomy site upon histological examination. Thermal ablation has the potential of being useful as well. Thermal ablation using radio frequency AC 
current is routinely used to treat bone lesions [149]. As well, laser ablation in the thermal regime has shown promise in applications of hemostasis [150], vascular stenosis [151] and cauterization [152], which could prove useful during osteotomy for reducing and/or stopping bone bleeds.

\subsubsection{Graphite as a topical chromophore}

In this chapter we explore the increase in efficacy of bone ablation at $1064 \mathrm{~nm}$ in the thermal regime using graphite as a topical chromophore. Since $1064 \mathrm{~nm}$ has a relatively low absorption cross-section for the various constituent materials of bone (ie. water, hydroxyapetite, collagen, etc.), most of the ablation response in the thermal regime happens after the surface incident to the beam has been heated enough for carbonization to begin. Once the black, carbonized tissue begins to form, the material removal process quickly accelerates due to the very large increase in light absorption by consequence. Before this, however, the time it takes for the carbonized tissue to first form (we refer to this as ignition time) is quite variable and unpredictable due to the inhomogeneity of bone tissue. Therefore, the use of a chromophore with a large absorption cross-section (similar to the carbonized tissue) as a topical absorber would significantly reduce the mean and variance of the ignition time. This is also important in isolating the effect of the gas-flow over the ablation site and reduce the effect of highly varying ignition times for the bone surface. Graphite was used as a topical chromophore for three main reasons:

1) High optical absorbance

2) Current use in many forms of bone surgery, where surgeons use sterile bone pencils to demarcate critical structures and cutting trajectories [153]

3) Ease of application via a simple pencil applicator 


\subsubsection{Coaxial assist gas}

Also in this chapter, we use a coaxial nitrogen gas jet to further increase the efficiency of ablation. Coaxial assist gas jets are commonly used in industrial laser machining applications. The gas flow helps to clear smoke, dross and plasma plume (assuming sufficient energy density) from the ablation site, allowing for incident photons to continue hitting the kerf bottom via an unobstructed path. Assist gas flow over the workpiece can also help in cooling the material and to mitigate the spread of heat damage and thermally induced cracking. Assist gas flow in the context of laser osteotomy is currently not well understood. Given the benefits seen in laser materials machining, the use of coaxial or blanket gas flow onto bone during laser ablation could have benefits such as increased etch rate and smaller heat affected zone (HAZ). Also, since pressurized gases such as nitrogen and oxygen are readily available in almost every operating room for multiple uses (anesthesia, insufflation, pneumatically actuated tools, etc.), the augmentation of laser osteotomy using assist gases for various surgical scenarios seems reasonable to investigate. This technique was first proposed by Wong et al. [154], where it was roughly determined which flow rates would be considered relatively safe in the context of neurosurgery. This was done by measuring the deflection of exposed sections of a porcine spinal cord (via laminectomy) using inline m-mode optical coherence tomography (OCT) whilst directly impinging the tissue with a nitrogen jet. The spinal cord was shown to compress almost $1 \mathrm{~mm}$ for a flow rate of 70SCFH, using a nozzle with an exit diameter of $3 \mathrm{~mm}$, and a tissue-nozzle separation distance of roughly $2 \mathrm{~mm}$. It was thought that any deflection beyond this point would induce unwanted somatosensory evoked potentials (SSEPs) and motor evoked potentials (MEPs). The study carried out in this chapter, which is a companion study to [154], investigates the effects that a nitrogen assist gas jet has on the etching efficacy of thermal bone ablation at $1064 \mathrm{~nm}$. The nozzle design and flow rate parameters were determined from insights gleaned from the results of [154]. We use a novel method of reducing variability in ignition behaviour of bone at this wavelength $(1064 \mathrm{~nm})$ by using a graphite topical absorber; this is the first time, to the knowledge of the authors, such a technique for bone ablation has been reported in literature. 


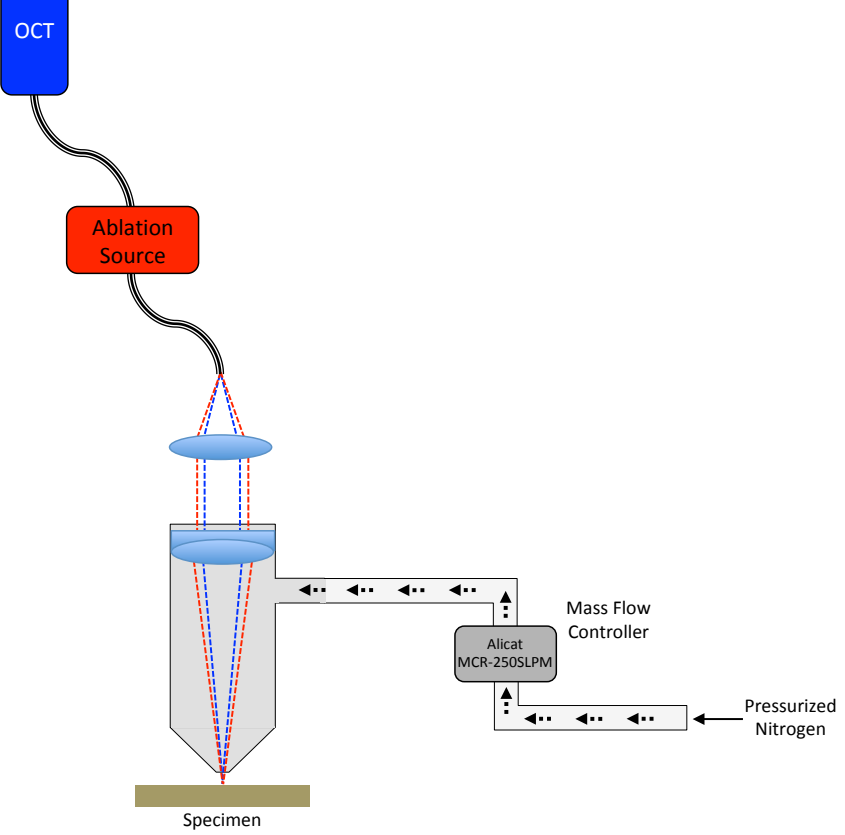

Figure 5.1: System diagram. The fiber laser system was built directly into the sample arm of the OCT based depth ranging system, allowing for real-time monitoring of the kerf bottom using m-mode imaging whilst ablating.

\subsection{Methods}

\subsubsection{Ablation laser and in-line Optical Coherence Tomography system}

The in-house built fiber laser system with integrated in-line OCT introduced in Chapter 2 was used in this experiment to ablate the samples and monitor progression of ablation craters in real-time $[9,89]$.

The 1064nm ablation laser light was produced by a high power fiber laser based on a design from [104]. It consisted of an all-fiber active cavity approximately 10m in length, constructed of Yb-doped fiber (Nufern LMA-YDF-10/130-VIII). Two fiber Bragg gratings (99.5\% and $10 \%$ reflective at $1064 \mathrm{~nm}$ for high and low reflectivity gratings, respectively) etched onto matching passive fiber (Nufern SM-GDF-10/125) were spliced onto the ends of the cavity with an APC connector terminating the output end. The cavity was pumped using a $976 \mathrm{~nm}$ fiber coupled laser diode (RealLight M976 $\pm 3-110-F 105 / 22-D 1)$ via a $(2+1) x 1$ high power fiber combiner (ITF 


\begin{tabular}{|l|l|}
\hline Center Wavelength & $1064 \mathrm{~nm}$ \\
\hline Average Power & $8.18-10 \mathrm{~W}$ \\
\hline Pulse Repitition Rate & $100 \mathrm{kHz}$ \\
\hline Peak Power & $523 \mathrm{~W}-1 \mathrm{~kW}$ \\
\hline Spot Size & $45 \mu \mathrm{m}$ \\
\hline
\end{tabular}

Table 5.1: Summary of fiber laser parameters.

\begin{tabular}{|l|l|}
\hline Center Wavelength & $1310 \mathrm{~nm}$ \\
\hline Bandwidth & $110 \mathrm{~nm}$ \\
\hline Average Power & $18.5 \mathrm{~mW}$ \\
\hline A-line Rate & $50 \mathrm{kHz}$ \\
\hline Axial Resolution (Theoretical) & $9 \mu \mathrm{m}$ \\
\hline
\end{tabular}

Table 5.2: Summary of OCT system parameters.

MM021112CC1A). All the fiber used in the construction of the fiber laser had a core diameter of $10 \mu \mathrm{m}$. The output from the laser was coupled from a pigtail fiber into a collimator (Thorlabs F280APC-C), then into a Thorlabs AC254-150-C achromatic doublet lens for focusing. The lens spacing between the collimator and focusing lens was approximately $2.5 \mathrm{~cm}$. The focal length was approximately $14.5 \mathrm{~cm}$.

The OCT system consisted of a 1310nm centered swept wavelength MEMS laser (Santec HSL20-50-S) with a bandwidth of 110nm, sweep rate of 50kHz (single-sided sweep, 62 percent duty cycle) and average power output of $18.5 \mathrm{~mW}$. An integrated Michelson interferometer (50/50 coupling ratio) with balanced detector system was used (Thorlabs INT-MSI-1300). The entire OCT system was constructed using SMF-28 fiber. The reference arm was constructed using a Thorlabs F280APC-C fiber-coupled free space collimator. The MEMS laser provides a k-clock output for sampling trigger.

We refer the reader to $[9,89]$ for details on the amalgamation of the OCT and fiber laser systems for inline ablation monitoring. 


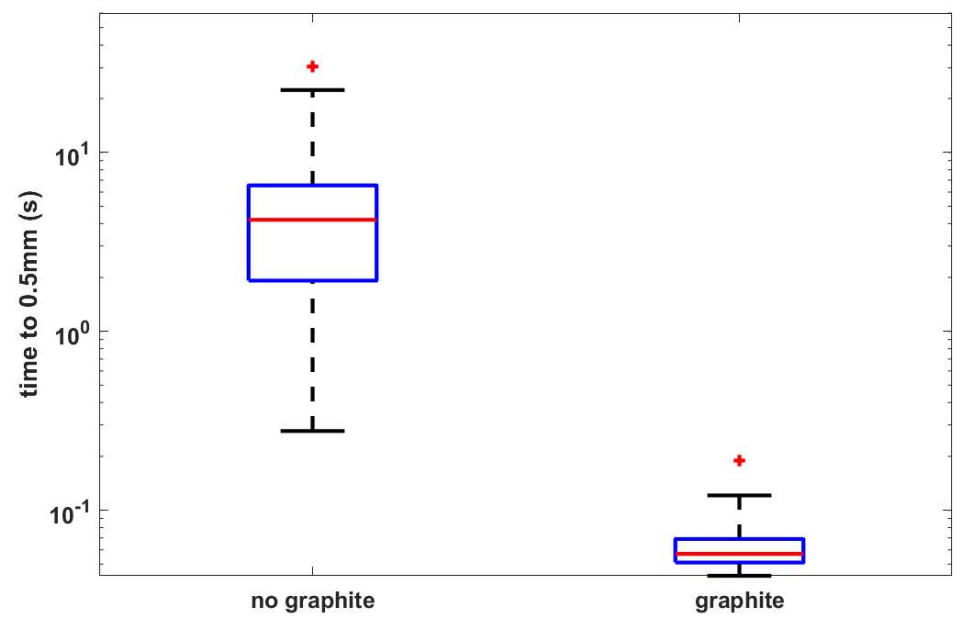

Figure 5.2: Results of ablation times for $0.5 \mathrm{~mm}$ depth with no graphite chromophore applied and with graphite chromophore applied to bone surface. We see a dramatic improvement in mean and variance of the time it takes to reach the target depth from when the laser was activated. Y-axis is in log scale.

\subsection{Experimental Results}

\subsubsection{Reduction of ignition-time variance using a topical chromophore}

Three fresh porcine scapula from two pigs were used in this experiment. The scapula was chosen in this experiment due to the similarity in structure to that of human cranial bone. Surrounding muscle and periosteum were initially left on the bone but were removed minutes before ablation experiments were conducted. Approximately twenty holes were percussion drilled into the surface of each scapula; 10 holes were ablated onto the bare bone surface and the remaining 10 after the application of bone pencil graphite onto the bone surface. Figure 5.2 shows the ablation results with and without topical application of graphite on bone surface prior to ablation. The time was measured between initiation of laser exposure and when the ablation crater reached a depth of $0.5 \mathrm{~mm}$ below bone surface. We see from the figure that there is a significant reduction in the mean and variance with the use of the topical chromophore. The mean time to $0.5 \mathrm{~mm}$ depth for no graphite used was 6.525 seconds with a standard deviation of 7.167 seconds, where as with graphite the mean value was found to be 0.068 seconds with a standard deviation of 0.029 seconds; both values showed and improvement of 2 orders of magnitude. 


\subsubsection{Gas flow experiments}

As mentioned earlier, the experimental setup was similar to that found in [154]. However, the key difference is that high-powered fiber laser mentioned in the previous section was placed inline with the OCT to monitor the ablation depth; this is summarized in Fig. 5.1. Graphite was used as a topical chromophore during these experiments as well, since from the results of the previous section it is reasonable to conclude that the use of graphite during gas assisted ablation is able to isolate the ablation results almost entirely as a function of the assist gas as large variances in ignition time and initial heating would be eliminated. For all the experiments conducted in this section, the following laser parameters were used: $P_{\text {avg }}=8.18 \mathrm{~W}, P_{\text {peak }}=523 \mathrm{~W}$, pulse repetition rate $=100 \mathrm{kHz}$. To ensure consistent flow rate of the assist gas, a mass flow controller specifically calibrated for nitrogen gas (Alicat Scientific MCR-250SLPM) was connected directly upstream of the nozzle to control the gas flow in real-time. The system was tested using MDF (medium density fiberboard) to confirm the behaviour. MDF was selected because it ablates easily and consistently at $1064 \mathrm{~nm}$. We see from the results shown in Fig. 5.3, the etch rate shows linear increase with respect to flow rate, but shows a plateauing and even decrease in etch rate for higher flow rate values. This result agrees well with the findings of Gabzdyl [42], showing that the system does behave as expected.

\subsubsection{Cortical bone experiments}

Four fresh porcine femurs were stripped of their periosteum tissue layers and cleaned with water. Porcine femurs were chosen for this part of the experiment due to the very thick layer of cortical bone (typically $>3 \mathrm{~mm}$ ), thus ensuring that accidental breach into the cancellous layer did not occur. Marks were made with the graphite pencil on the surface of each bone. The flow rates used with were $0,10,30,50,70$ and $90 \mathrm{SCFH}$ (standard cubic feet per hour); the exposure times were 250, 500, 750 and $1000 \mathrm{~ms}$. For each flow rate/exposure time combination, 10 holes were percussion drilled into the femurs, giving a total of 240 holes. The maximum achieved depths were analyzed immediately via b-mode imaging using a separate, calibrated swept-source OCT system. The results of this experiment are shown in Fig. 5.4. It can be seen that there is a significant drop in 


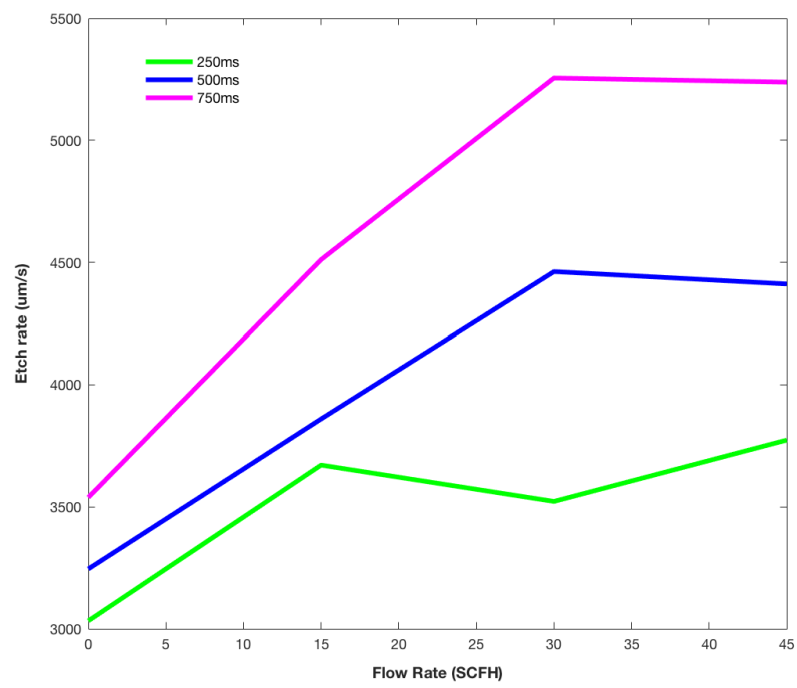

Figure 5.3: Effects of nitrogen flow during percussion ablation of medium density fiber board (MDF) for different exposure times.

etch rate after the $250 \mathrm{~ms}$ window; however there does appear to be an increase in etch rate with an increasing nitrogen flow rate. However, even though Fig. 5.4 does show that the etch rate increase does seem to be linear for the $250 \mathrm{~ms}$ exposure time, the behaviour seems non-linear with respect to flow rate for the other exposure times. The data was fitted to a least squares $2^{\text {nd }}$ order polynomial model with a coefficient of determination of $R^{2}=0.98$, suggesting a good fit. Below is the fitted polynomial describing the behaviour:

$$
\nu_{\text {etch }}=-0.0357 \nu_{\text {flow }}^{2}+0.002599 \tau_{\text {ex }}^{2}+11.57 \nu_{\text {flow }}-5.731 \tau_{\text {ex }}-0.008063 \nu_{\text {flow }} \tau_{\text {ex }}+4243
$$

where $\nu_{\text {etch }}$ is the etch rate in $\mu \mathrm{m} / \mathrm{s}, \nu_{\text {flow }}$ is the nitrogen flow rate in SCFH, and $\tau_{e x}$ is the exposure time in milliseconds. To further investigate the effect of gas flow on the etch rate of cortical bone, the same procedure was used to drill holes into three more porcine femurs. This time, the m-mode data from the OCT system inline with the fiber laser (see figure 5.1) was recorded to monitor the kerf bottom as a progression of time during ablation. For these experiments, only the nitrogen flow rate was varied; 10 holes were percussion drilled into the femurs for each flow 


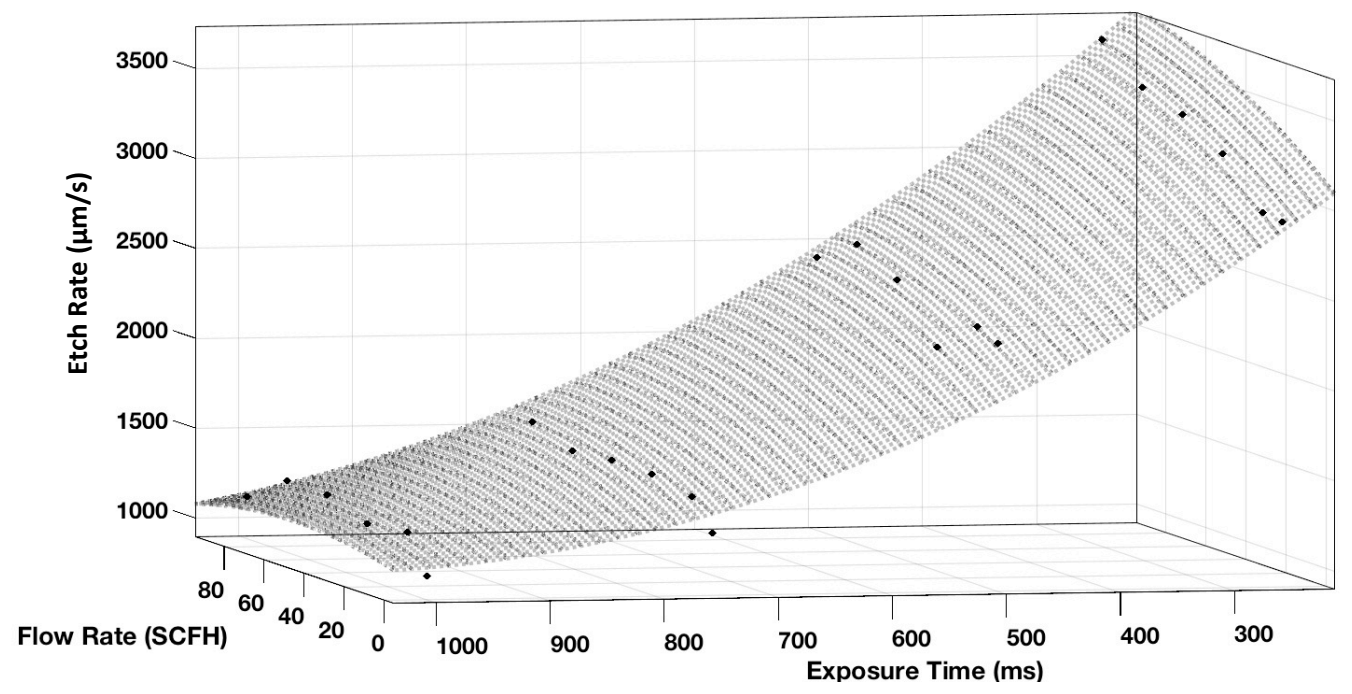

Figure 5.4: Mean etch rate as a function of exposure time and flow rate. Etch rate is quite high during the smallest exposure window and highest nitrogen gas flow. Etch rate is seen to increase as a function of flow rate specifically, but an increase flow does not linearly translate to increase in etch rate.

rate. Figure 5.5 shows the means of each set of flow rate trials. It was found that the assisted gas flow for up to 30SCFH showed a clear improvement in the depth achieved, but beyond that the improvement was reduced to near no-flow condition. It was also found that higher flow rates did result in a slight increase in slope of the m-mode progression (corresponding to the etch rate) during the initial, nearly linear portion.

\subsection{Discussion and Conclusion}

It was found that the mean time of exposure to reach a crater depth of $0.5 \mathrm{~mm}$ was significant between holes that did and did not have topical application of graphite. For the samples where no graphite was used, typically a very long period of heating of the cortical bone surface was seen before rapid material drilling could take place. This is thought to be due to the relatively poor absorption of $1064 \mathrm{~nm}$ light in osseous tissue. Due to the non-homogeneity of bone, these periods were shown to have an extremely high variance; this is shown in Fig. 5.2. Using a poorly absorbing wavelength in conjunction with a topical chromophore to cut bone can have the advantage of isolating ablation activity solely to regions where and in close proximity of chromophore application. This way, a margin of safety can be realized so that inadvertent ablation due to unexpected/sudden beam shift (ie. getting bumped or knocked) would be less likely to occur. As well, the use of a 


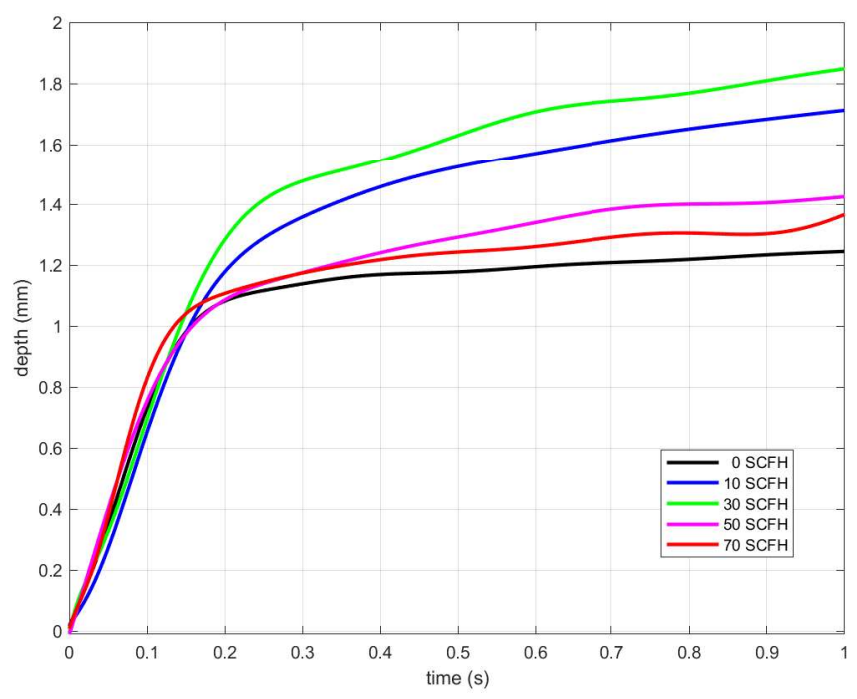

Figure 5.5: Time progression of depth measured via inline m-mode OCT, as a function of flow rate. $30 \mathrm{SCFH}$ is shown to most dramatically improve ablation. 70SCFH is shown to improve slope initial ablation progression, just before $200 \mathrm{~ms}$.

chromophore can enhance the use of an overall lower-power laser, thereby adding another aspect of safety in case of failure or damage to delivery fiber.

It was observed that etching efficiency does reduce with respect to increase in exposure time. This could be because of the kerf bottom being out of the focal region of the laser for extended periods of ablation. However, it was shown in this chapter that for 30SCFH, the efficacy of ablation did increase for the same approximate exposure time. Generally, it is desirable to keep exposure time limited for a given volume to reduce unwanted heat effects; therefore, it is crucial to optimize etching efficiency to achieve maximal penetration in the minimal amount of time.

The results reported in this chapter show similar findings to that reported by Aljekhadab et al. [155] where an increased etch rate was reported with the use of airflow over the ablation sample. As well, in this chapter it is also demonstrated cleaner and more well defined edges of craters compared to water-layer assist and no assist fluid. The use of nitrogen has some key advantages in its potential use as an assist agent in ablation over other gases and liquids. Since most of the atmospheric air is composed of nitrogen, there is no practical need to optically compensate for change in refractive index, as would potentially be the case if an optically dissimilar gas or liquid 
was to be used. This also means that unwanted absorption or lensing through the assist medium is eliminated. The use of nitrogen also suffocates the ablation region of oxygen, reducing the chance of flare up. As well, Nitrogen is readily available as a standard medical gas in operating rooms, therefore this does not have to be piped from outside the operating or brought in using a pressurized cylinder. We see the relationship in Fig. 5.4 between flow rate and exposure time vs etch rate to be strongly second order. We hypothesize that even though the nitrogen jet used in this study is a of relatively low Reynold's number, turbulence at the mouth of the kerf increases with an increase in flow rate, thereby decreasing the laminar flow entering the kerf. This is thought to lead to a decrease in clearing efficiency of smoke and ejected debris during ablation. The use of a coaxial nitrogen jet can have some drawbacks. It has the potential to cause surface drying of the bone, which could lead to undesirable effects such as increased cell death and localized heating. As well, pressurized fluid flow may cause unwanted spattering of fluids; whether this would be worse than the use of a high-speed spinning drill bit is a matter of future study.

It has been noted by several studies that ablation of bone, specifically in the thermal regime, causes a delay in healing post laser osteotomy compared to traditional mechanical methods [156, 157, 158]. Although this study did not quantify the thermal damage conducted into the tissue as a result of laser energy deposition, the proposed techniques have the potential to mitigate some of this damaged-induced healing delay primarily in two ways. First, the use of a topical absorber such as graphite can dramatically reduce the exposure time of the bone to the ablation light, thereby reducing the overall time that excess heat can conduct into surrounding tissue. Second, inert assist gas flow may act to locally cool the area, potentially reducing the heat-affected zone around the ablation site. The hope of this study and such explorations is to help make low-cost, effective laser surgery more prevalent in clinical practice. 


\section{Chapter 6}

\section{Dynamic Axial Correction of Payload using OCT}

The work presented in this chapter resulted in the following journal publication [159]:

Jivraj, Jamil, Chaoliang Chen, Dexter Barrows, and Victor XD Yang. "Optical coherence tomography for dynamic axial correction of an optical end-effector for robot-guided surgical laser ablation.” Optical Engineering 58, no. 5 (2019): 054106.

\subsection{Introduction}

In this chapter, the hardware and software techniques introduced in the previous chapters are used to develop a method for the robot to autonomously correct for end-effector deviation along the target surface using OCT. Maintenance of the beam waist at the surface of the bone in real-time is critical for optimal ablation quality. The challenge is that unlike in most applications in industrial laser material processing, most bones do not have large surfaces that are smooth and flat. As well, minute variations along the surface that can still affect ablation quality are not always fully captured using current medical imaging modalities given resolution constraints; this means that adaptation along a surface using a-priori knowledge of surface variations may not be fully affective. Therefore, it would be quite advantageous for a laser osteotomy system to make continuous, realtime, sub-millimeter adaptations along the optical axis in order to maintain beam focus along 
uneven surfaces.

Several techniques exist in for laser materials processing to maintain focus of the workpiece [160]. Field effect sensors, and contact sensors have been demonstrated with success in the processing of certain materials. However, in the context of surgery, these sensors can not perform adequately. Field effect sensors rely on inductive or capacitive interaction between the workpiece and the sensor to determine axial distance. These methods work for metallic work pieces, but they can only be used between laser pulses since the high intensity of the electric field of the laser pulse affects distance measurements. Contact sensors would not be suitable for applications of laser tissue ablation because of their innate negation of the non-contact benefit of using laser light to cut tissue. Various optical sensing methods have been reported to measure workpiece distance along the optical axis in real-time. Techniques involving monitoring of the laser spot diameter using image sensors have also been developed [161, 162], but may not perform well on irregular surfaces, defocusing direction may remain ambiguous, and marking the specimen by firing the machine beam in order to make a spot measurement may cause unwanted damage to the target. Hand et al. developed a technique that redirected a fraction of the process-generated light into a separate optical path for analysis of the chromatic aberrations to determine focal error [163]. Cao et el. recently developed methods involving a defractive beam samplers[164], as well as hole-mask, separate interrogation source $[165,166]$ and multiple intermediate bulk-optic elements and a CCD to place the target in the focus of the machining beam. By monitoring the change in diffraction pattern on the $\mathrm{CCD}$, these techniques allow for determination of the workpiece position along the optical axis and the direction of defocusing.

We explore the use of OCT to guide a robot-guided laser ablation objective payload over a target for surgery. OCT has been used to guide other types of surgical payloads. Studies have been carried out by Yu et al. [167, 168, 169, 170] for OCT guidance of retinal surgery payloads. B-mode OCT feedback was used to track the tips of various instruments (micropipette[170], grippers [169], injection pipette [168]), in real-time. Sub-millimetre tracking accuracy was reported whilst using a Steward-Gough robotic platform. Cheon et al. explored a similar application in ophthalmic surgery where OCT was used to guide a needle to a precise depth into a bovine eye [171]. These studies 
prove that OCT can be successfully used in real-time guidance of various robotic surgical payloads.

In this chapter the use of m-mode OCT as a feedback mechanism for dynamic axial adjustment of the payload of a 7-DOF robot with respect to uneven and rough/non-planar surfaces is proposed. The peak of the OCT signal, which defines the surface, is used for correcting the offset distance along the optical axis between the robot-mounted objective lens and surface of the target. Given that OCT innately has the ability to measure displacement along the optical axis, ambiguity in defocusing direction is removed using this method. The application of this technique is for ablation of bone during surgery via robotic path guidance. The technique proposed in this chapter can be easily integrated into various surgical and non-surgical ablation schemes. Figure 6.1 shows two widely reported schemes of dual-wavelength beam combination that has been used for ablation process monitoring and control using OCT. a) shows the most prevalent beam combination method, which uses a dichroic element to guide both free-space beam paths into a single set of objective optics $[172,99,173]$. b) shows a method that maintains a single fiber output path for both beams by combining the ablation source (in this case a fiber laser) directly into the sample arm of the OCT system, reducing the amount of bulk optic elements at or near in the output [9]. Both methods can be easily used to design an optical payload for robot-guided ablation, as shown in figure 6.2. It is important to note that any method that uses different wavelengths for ablation and interrogation with a single set of objective optics will have a a shift along the axial direction between the waists of both beams. This easily measurable offset must be taken into account so that the ablation beam waist is always kept at the specimen surface.

\subsection{System description}

\subsubsection{Hardware}

robotic manipulator

The robotic manipulator used in this system is the IIWA, manufactured by KUKA AG. The IIWA is a 7-DOF anthropomorphic manipulator designed to work in close proximity with humans, such as in a surgical setting. The robot has a quoted maximum payload weight of $14 \mathrm{~kg}$ and a positioning 
a)

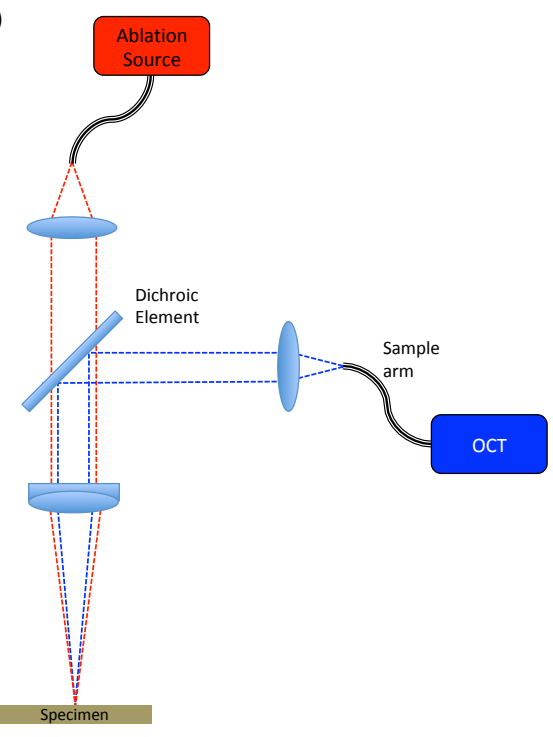

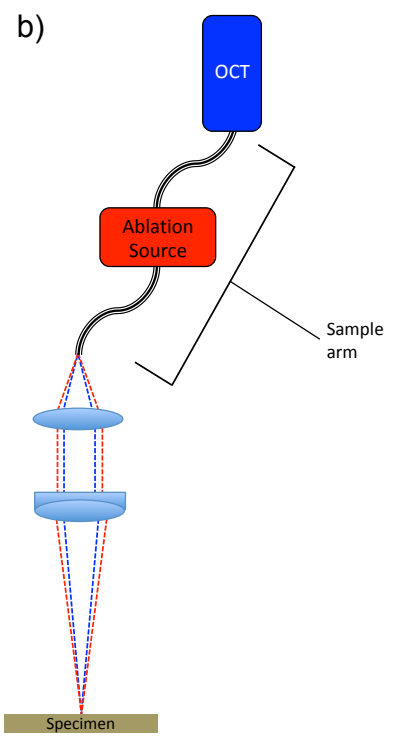

Figure 6.1: Methods for ablation and OCT beam combination. Image a) shows the most common method, using a dichroic mirror to combine the high-powered ablation beam with relatively low-powered OCT beam. The wavelength-specific transmissivity of the dichroic element prevents high-powered ablation light from following the beam path of the OCT system. b) shows a method that involves a fiber laser to be built directly into the sample arm of the OCT system, as proposed in [9].

accuracy of $\pm 0.1 \mathrm{~mm}$ [174]. The robot was sent axial position information in real-time via ethernet communication protocol using a desktop computer. The update period between the robot and the computer was approximately 500ms.

\section{Optical Payload and Beam Delivery}

The sample arm beam of the OCT system is delivered via fiber to a Thorlabs F810APC-1064 doublet collimator. The collimated light is then focused through a Thorlabs AC-254-150C achromatic doublet lens. The measured spot size $d_{o}$, depth of focus $L_{d}$ and focal length $L_{f}$ is $48 \mu \mathrm{m}, 2.1 \mathrm{~mm}$ and $146 \mathrm{~mm}$, respectively; figure 6.3 shows a physical summary. The focusing optics are attached to the mounting flange of the robot (corresponding to the seventh degree of freedom) using an optical breadboard. The location of the focal spot is programmed to be the tool center point (TCP) of the end-effector. 


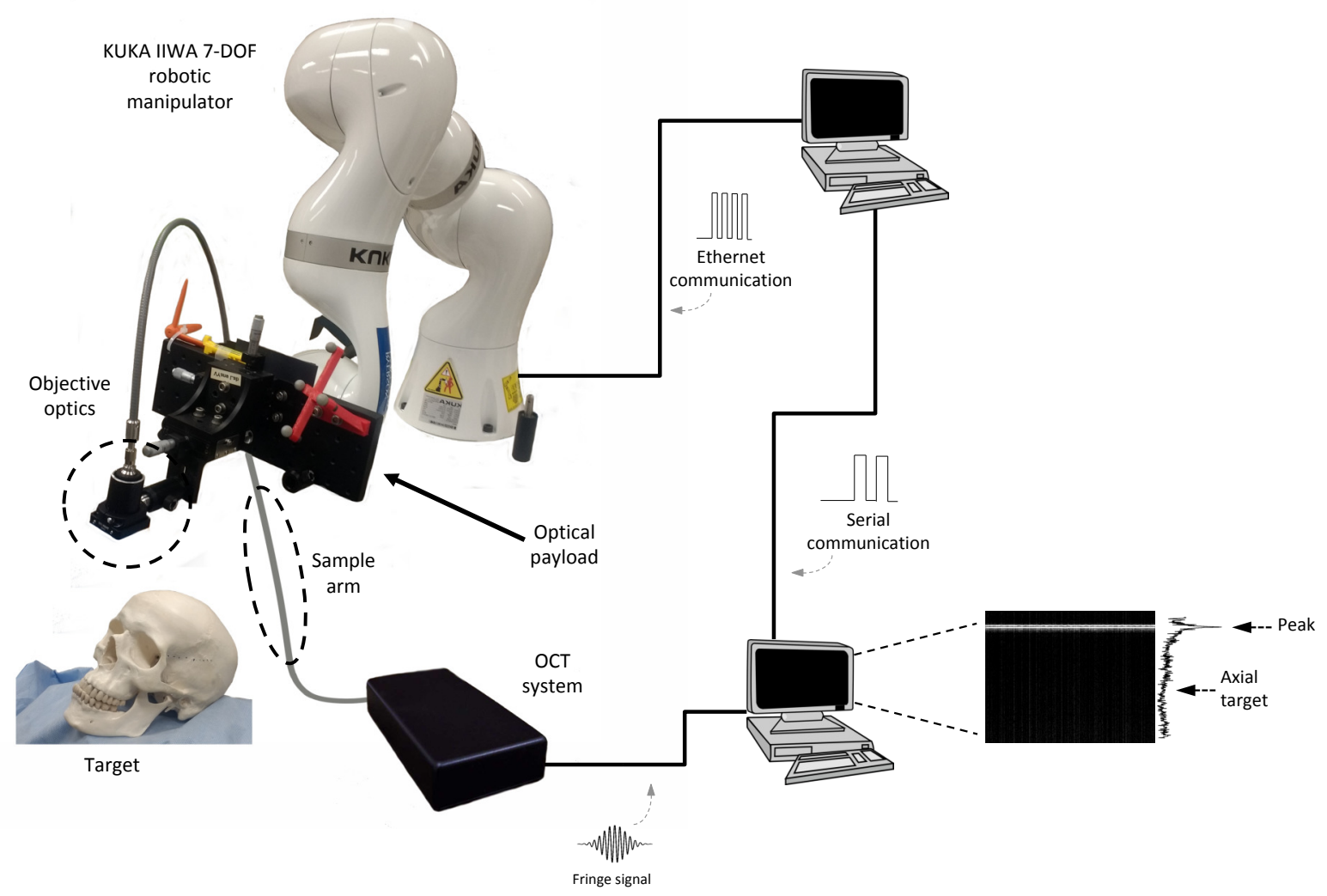

Figure 6.2: System diagram. The 7-DOF manipulator carries optical payload (with objective optics) over target. Feedback of axial position over target's surface is measured in real-time via OCT and correction is sent to the robot over high-speed serial communication. The two elements highlighted in dashed lines (objective optics and delivery fiber) show where either of the two method of beam combination explained in figure 6.1 would take place.

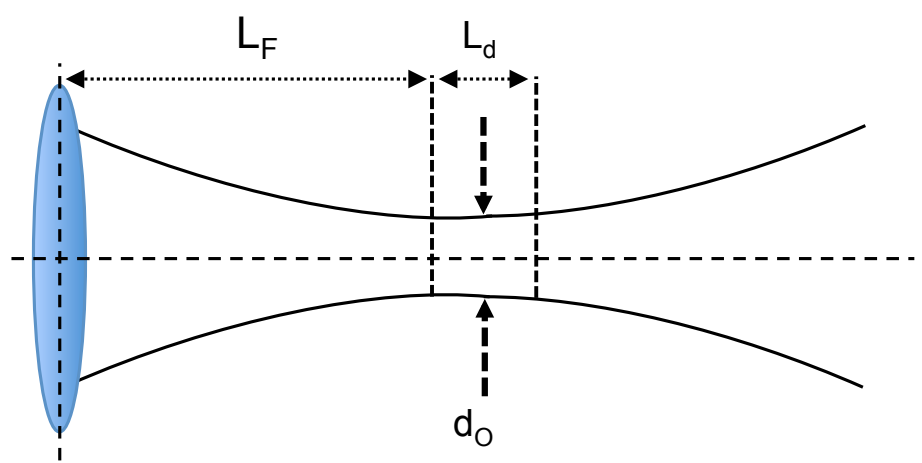

Figure 6.3: Anatomy of a focused gaussian beam. Depth of focus denotes planes along optical axis at which beam area is twice that of the area at beam waist. $L_{d} / 2$ denotes Rayleigh length. It is typically desirable to maintain the specimen surface at the beam waist. 

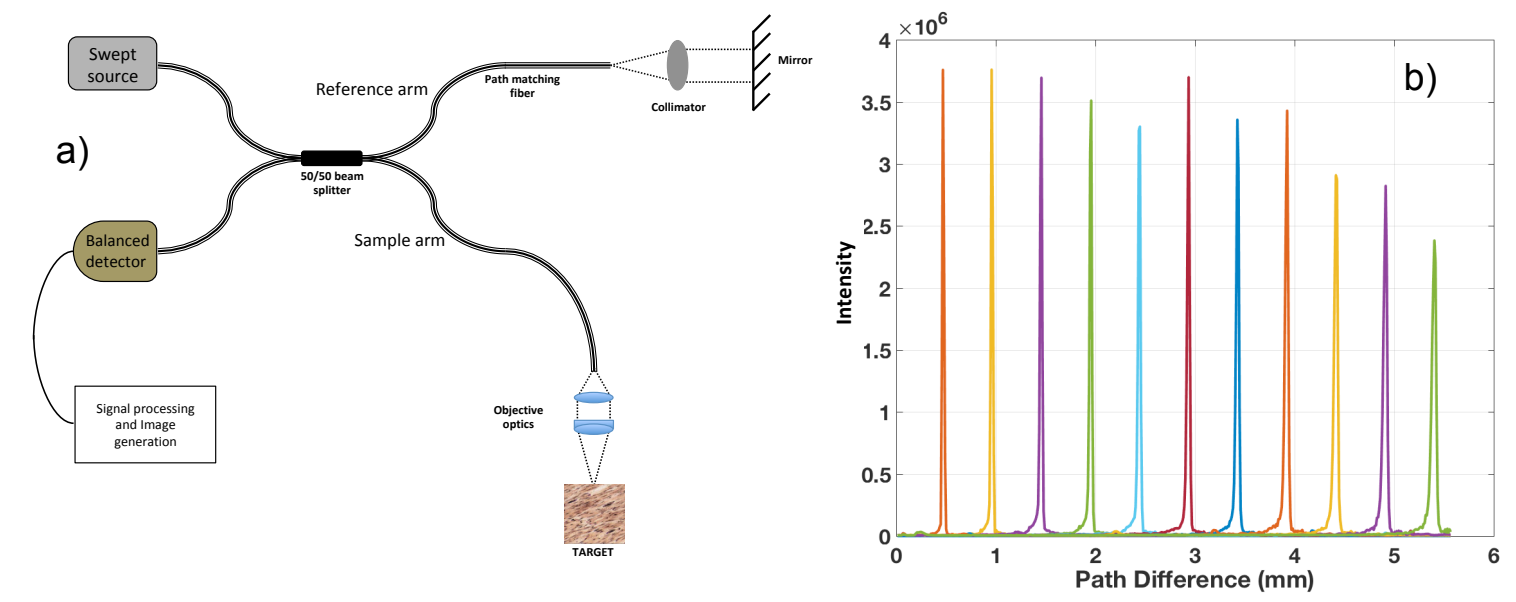

Figure 6.4: a) Schematic of fiber based swept-source OCT system. b) Point spread function (PSF) of OCT system. Axial resolution $=9 \mu \mathrm{m}$.

\section{Swept-source OCT system}

The OCT system consisted of a 1310nm centered swept wavelength MEMS laser (Santec HSL-20$50-\mathrm{S}$ ) with a bandwidth of $105 \mathrm{~nm}$, sweep rate of $50 \mathrm{kHz}$ and (measured) average power output of 18mW. A fiber-based Michelson interferometer (50/50 coupling ratio) and a Thorlabs PDB415C $100 \mathrm{MHz}$ balanced detector was used. The signal was acquired using an Alazartech ATS9350 digitizer card. The entire OCT system was constructed using SMF-28 fiber. Figure 6.4 a) shows the diagram for the OCT system and $6.4 \mathrm{~b}$ ) shows it's point spread function (PSF). Peak detection of the Fourier transformed interference fringe signal (acquired by the balanced detector) was used to determine the axial offset of the surface of the target; this information was processed in real-time using a desktop computer using in-house developed software.

\section{Explanation of elimination of de-focusing direction ambiguity}

Given the nature of the swept-source OCT interference fringe signal captured by the photodetector, de-focusing directional ambiguity is eliminated. The frequency composition of the fringes is depthdependant - higher frequencies are proportional to larger optical path difference. Figure 6.5 shows this concept in greater detail. The plot in the diagram shows the fringe signal after the Fourier 


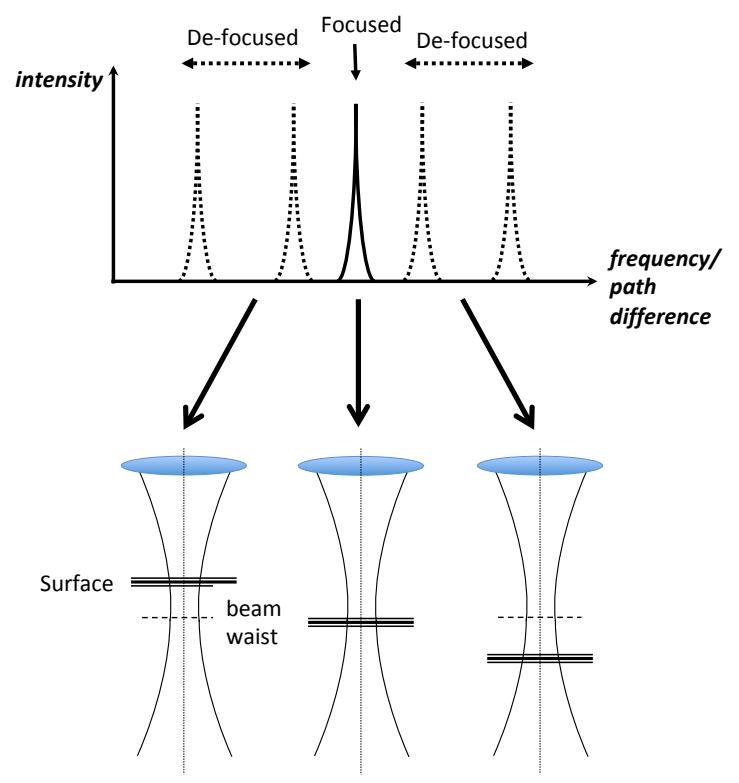

Figure 6.5: Elimination of ambiguity in de-focusing direction. The plot represents the fringe signal postFourier transform. The peak represents the position of the target surface along the optical axis. The frequency is directly proportional to the optical path difference, therefore position of the peak along the frequency axis represents the absolute position of the surface along the optical axis.

transform; the sharp peaks represent the surface reflection. The further the peak travels along the frequency axis, the larger the optical path difference, meaning the further the surface is away from the payload along the optical axis. Any point along the frequency axis can be arbitrarily chosen as the target position; typically this is where the beam waist is placed in relation to the target. Any deviation from this chosen position can be easily compensated for by determining the difference between the current position along the frequency axis and the desired, focused position.

\section{System Integration}

A high-speed serial communication link between the two desktop computers was established so that OCT-acquired axial peak position could be relayed in real-time to the computer commanding the manipulator. The workflow for the system was designed as follows:

1. Acquire interferogram from the photodetector. 
2. Perform signal filtering and Fourier transform to attain depth-dependant frequency spectrum.

3. Perform peak detection and check if the peak has moved from initial position (taken to be $0 \mathrm{~mm}$ ); if peak has moved, send robot the updated axial correction to bring it back to $0 \mathrm{~mm}$ position.

\subsection{Experiments and Results}

A series of experiments were performed in order to characterize the mean distance at which the robot was able to position the beam waist relative to the surface of a target whilst the target was linearly translated. The goal was to maintain the surface of the target within the depth of focus, as close to the beam waist as possible. The axial positions were recorded in real-time using m-mode OCT imaging.

\subsubsection{Step response}

The robot was made to adapt to a step elevation change of $2 \mathrm{~mm}$, which represents the most aggressive input change possible; with the m-mode system response shown in figure 6.6. The figure shows the surface at axial error at $0 \mathrm{~mm}$, meaning that the surface is at it's intended target axial position. The target surface is dropped $2 \mathrm{~mm}$, showing the negative step change in figure 6.6 ; after a short dwell period, corrective action is initiated and the robot end effector is moved along the optical axis until the surface of the target is brought back to $0 \mathrm{~mm}$ axial error. The process is then repeated with a positive step change.The total response time (10\% - 90\% definition) of $1.3 \mathrm{~s}$ was found, which corresponds to a total response rate of $0.77 \mathrm{~Hz}$.

\subsubsection{Axial correction on sloped progression}

To characterize axial adaptation performance along a gradual elevation change, a surface sloped at various angles was used as a target, as shown in figure 6.7. The optical payload was linearly translated $10 \mathrm{~mm}$ laterally at four different linear velocites $(0.5 \mathrm{~mm} / \mathrm{s}, 1.0 \mathrm{~mm} / \mathrm{s}, 1.5 \mathrm{~mm} / \mathrm{s}, 2.0 \mathrm{~mm} / \mathrm{s})$ relative to a surface at three different slopes $\left(10^{\circ}, 20^{\circ}, 45^{\circ}\right)$. M-mode OCT data was recorded to show axial deviation from the target similar to figure 6.6; this is sown in figure 6.8). As expected, 


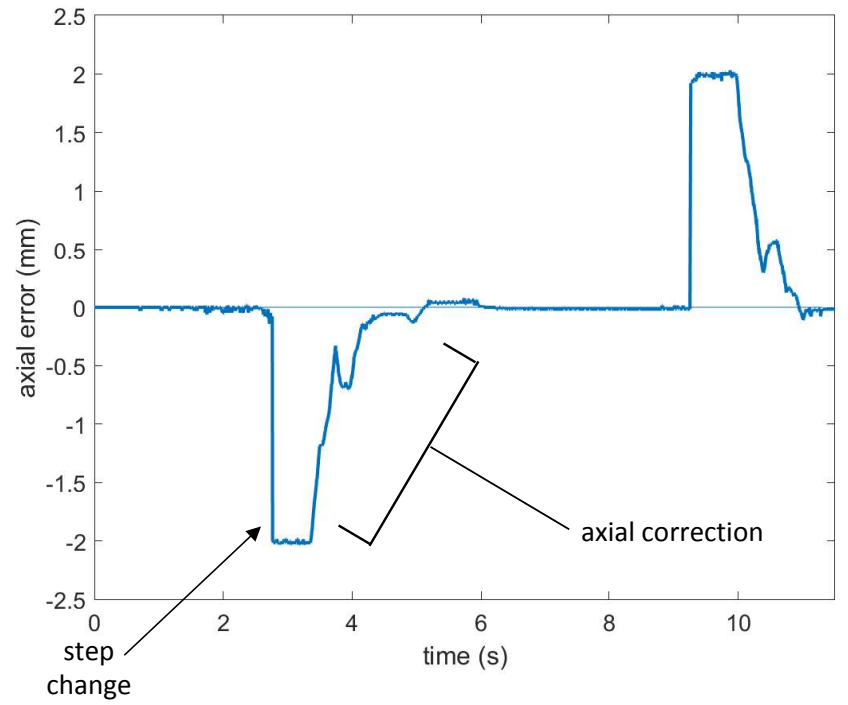

Figure 6.6: Response of system correction to step change in the axial direction.

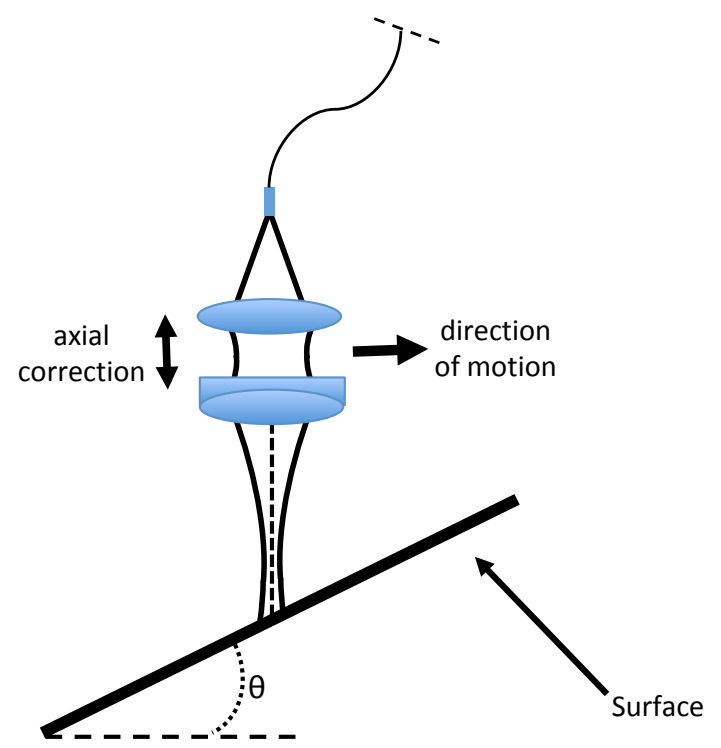

Figure 6.7: Schematic of slope progression experiment. $\theta$ represents the angles that the surface was positioned to. 

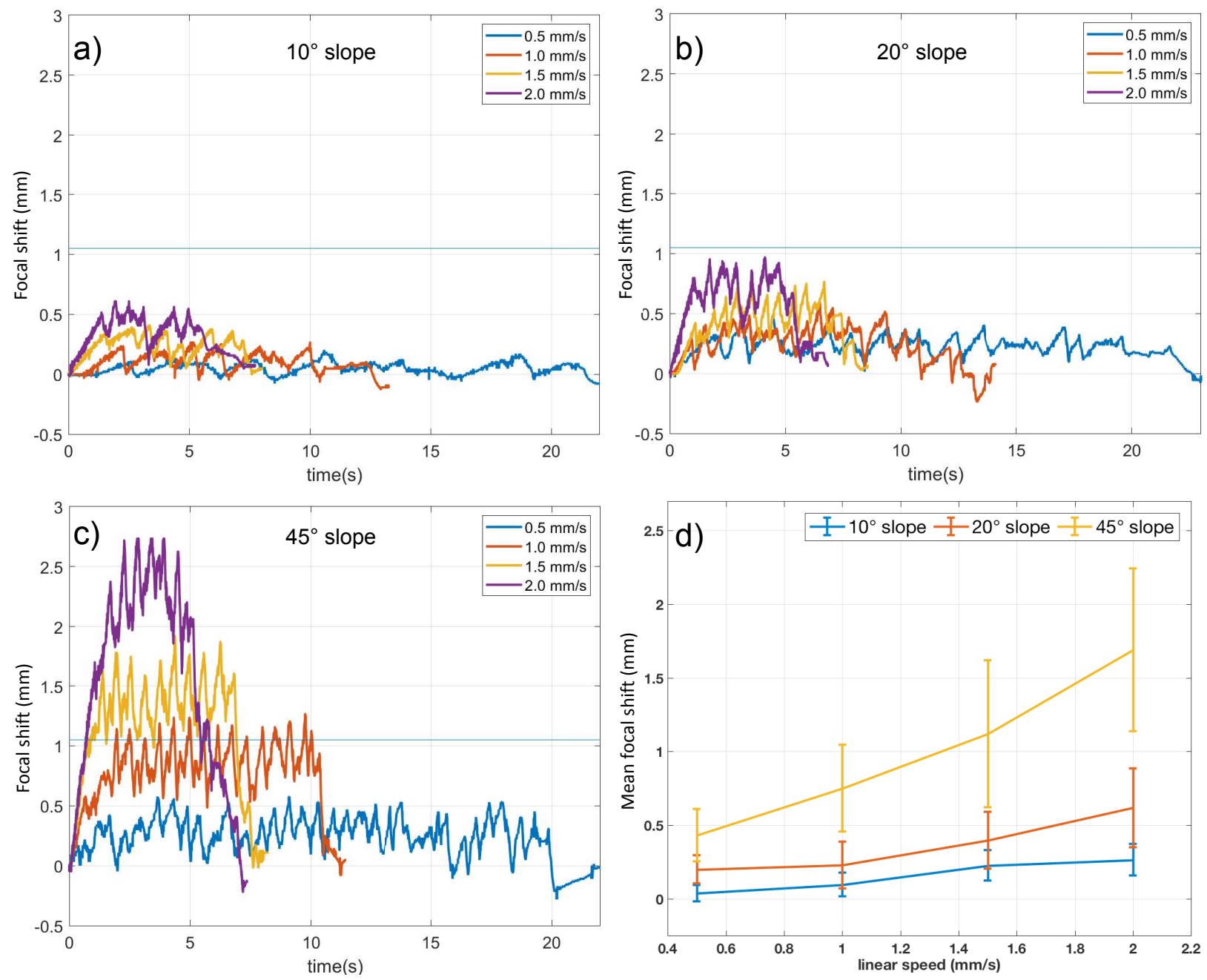

Figure 6.8: Phantom experiments summarizing tracking error versus linear speed and slope. Panels a), b) and c) show m-mode tracking error at 10, 20 and 45 degree surface incline, respectively. Blue line demarcates Rayleigh length. d) shows mean of tracking error experiments.

the increase in slope angle and linear velocity corresponded to a larger cumulative axial error. It was also seen that at the most aggressive slope, $45^{\circ}$, linear speeds of up to $1.0 \mathrm{~mm} / \mathrm{s}$ could be maintained with the axial error still staying below the Rayleigh length of the focusing optics. At $1.5 \mathrm{~mm} / \mathrm{s}$ and higher, the axial error began to drift outside the Rayleigh length.

\subsubsection{Experiment on skull phantom}

A 1:1 scale human skull phantom was used as a target to characterize the mean axial tracking error. This allowed for a very close approximation of the system performance characterization for a real world osteotomy scenario.The phantom was placed such that the transverse plane of the skull was 

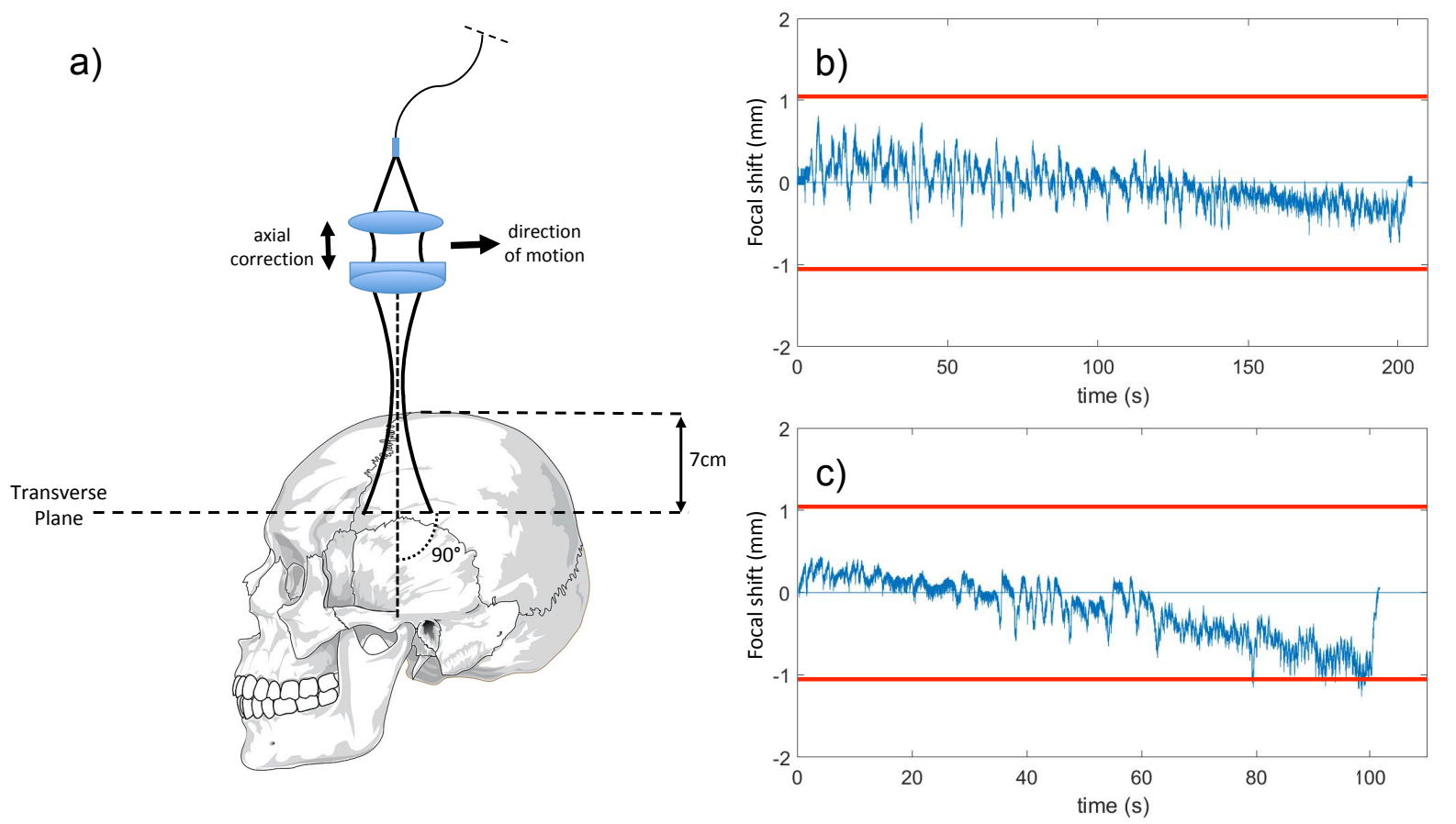

Figure 6.9: a) Diagram showing axial adaptation on a skull phantom target. Experiment is executed over the transverse medial suture. Total axial height change is $7 \mathrm{~cm}$ over the $10 \mathrm{~cm}$ linear translation. b) and c) Axial tracking error results over transverse suture of the skull phantom for $0.5 \mathrm{~mm} / \mathrm{s}$ and $1.0 \mathrm{~mm} / \mathrm{s}$, respectively. Red lines represent Rayleigh lengths. 
positioned perpendicularly to the optical axis of the objective lens, as shown in figure 6.9. The payload was moved along the sagittal suture, simulating a cranial osteotomy. The mean tracking error from the beam waist was found to be $-0.02 \mathrm{~mm}$ and $-0.2 \mathrm{~mm}$ for $0.5 \mathrm{~mm} / \mathrm{s}$ and $1.0 \mathrm{~mm} / \mathrm{s}$ linear velocity, respectively.

\subsection{Discussion and Conclusions}

We have shown that OCT is a viable method for surface detection and dynamic re-focusing using a robotic manipulator. The technique developed is able to keep the target within the depth of focus for the objective lens at surface inclines as aggressive as $45^{\circ}$ for linear velocities of up to $1.0 \mathrm{~mm} / \mathrm{s}$. For less aggressive slopes, dynamic focusing was successful for linear velocities of up to $2.0 \mathrm{~mm} / \mathrm{s}$. This agrees well actual bone cutting feed rates, such as those explored by Davidson et al. [175] (up to $5 \mathrm{~mm} / \mathrm{s}$ ). This technique can be useful when the surface profile is not known a-priori, or if the surface tends to shift during ablation, i.e. during patient breathing. As well, this technique is able to maintain focus along a surface with variations that are irresolvable using most medical imaging modalities. The simplicity of this technique lends well to integration into existing ablation systems where OCT is integrated in-line for depth monitoring [172, 143, 9, 89]. As well, it has

been shown in this chapter that this type of correction is possible without the use of galvo-scanners for b-mode scanning, thereby reducing potentially maintaining a minimum payload complexity to a single collimating lens and single objective lens.

As seen in section 3, mean accuracy during increasingly aggressive slope of the surface significantly improved with the reduction of speed. This means that the axial error can be significantly improved if the feed-rate of the workpiece can be dynamically modulated based on axial deflection/incline as ascertained by OCT surface feedback.

Future steps in this work entail increasing the system loop-rate so that adaptation can occur faster, thereby potentially adapting to high-frequency surface artifacts and increasing the feed rate of the workpiece. 


\section{Chapter 7}

\section{Summary, Conclusions and Future Work}

Lasers have had a relatively slow uptake in medical applications compared to the promise that researchers saw during the infancy of this technology. Contrasting this to the sheer dominance of laser technologies in the areas of telecommunications and manufacturing, there is much left to be desired in terms of laser-based revolutions in medicine. Fortunately, there does exist some sparse examples of important and prolific laser-based advancements in medicine. Laser interferometric techniques introduced in the late 1980's and early 1990's [176, 177, 178, 179, 180] culminated in the development and dominance of OCT in not only ophthalmology, but increasingly in various other subspecialties of medicine including dermatology [181, 182, 183], cardiology [184, 185, 186], and gastroenterology [187, 188, 189]. OCT represents one of, if not the strongest example of the potential of lasers to revolutionize medicine. In the same spirit, lasers have the potential to dominate in osteotomy procedures. The challenge is to create laser-based tissue cutting systems that do not rob the surgeon of depth feedback, and are intuitive enough to interact with such that specialized training and complex setup is mitigated as much as possible.

The following scientific and engineering contributions were made throughout this dissertation in the fields of robotic surgery and laser osteotomy:

- The integration of a high-powered fiber laser with coaxial inline OCT for depth guidance through an all-fiber beam path, and with the largest reported ablation laser power to sweptsource power ratio ( greater than $2 \times 10^{4}$ peak to average power). An alternate method was 
presented using bulk optics instead of fiber gratings to achieve the same functionality of ablation monitoring.

- The first to observe and report the phenomenon of seemingly optical path difference (OPD) dependant PSF peak broadening, actually due to k-clock mis-sampling caused by abnormally large optical path length (OPL). Two solutions were realized in this dissertation: 1) using an MZI-based sampling clock with equal OPL, thereby inducing an equal delay and 2) delaying the original k-clock signal though electrical amplification and subsequent propagation through a large length of coaxial cable.

- The first to achieve trajectory generation and end-effector orientation of a 7-DOF robotic manipulator using simple penscription and structured light imaging. This was then applied to laser osteotomy, also demonstrated for the first time.

- The first to demonstrate the use of a topical chromophore (in this case, graphite) to improve bone ablation at $1064 \mathrm{~nm}$ by two orders of magnitude in terms of mean and variance of ablation time.

- The first to demonstrate the enhancement of bone ablation at $1064 \mathrm{~nm}$ using a coaxial nitrogen assist gas jet.

- The first to demonstrate the use of OCT for real time, sub-millimeter axial correction of the end-effector of a robotic manipulator in order to maintain beam focus (within rayleigh range) along the surface of an ablation target.

\subsection{Future Work}

The areas of robotic neurosurgery and laser osteotomy still remain fruitful for exploration, understanding and application. The work presented in this dissertation has lead to some interesting and potentially impactful lines of inquiry. Some of these questions are discussed below. 


\section{Robust OCT detection of bone-endostium interface during ablation}

Leung et al. demonstrated the successful pre-breach detection of a bone-air interface using inline OCT during percussion drilling [100]. Zhang was able to demonstrate similar results during laser cochleostomy [63]. However, it remains to be seen if this can be replicated consistently and with slim margins of error for applications in large osteotomies (ie. bone flaps, mastoidectomy, etc.), especially during blood obfuscation and heavy formation of carbonization. If this is achieved, then the possibility for using OCT-guided osteotomy without pre-surgical registration to a CT scan has the possibility of becoming a clinical reality. Successful, quantitative awareness of approaching full breach of the proximal cortical bone layer of any bone would approximate, if not surpass, the feel surgeons currently use during bone drilling to ascertain how deep the cutting instrument has travelled.

\section{Reduction of roundtrip OCT power loss through fiber laser}

As mentioned before, much of OCT power loss through the fiber laser was due to the poor guidance capability of SM-GDF-10/125 around 1310nm. Future designs of the system could have improved SNR by using fiber better suited to guide $1310 \mathrm{~nm}$ light (though that would necessitate a change in fiber laser design), or to use a different wavelength for OCT interrogation that is able to be guided well and is out of the rejection bandwidth of the TFBG coupling scheme.

\section{Advanced penscriptive cues for diverse robot behaviour}

In this dissertation it was demonstrated that the use of penscription is definitely a possibility for human-computer (more specifically, surgeon-robot) interaction. Robotic guidance of straight line and closed/pseudo-circular penscribed trajectories was successfully demonstrated. Further research can be done in not only having the system recognize trajectories, but also to recognize penscribed cues for various behaviours such as:

- Arrows for cutting directions and feed rate/speed

- "X's" for safe/danger zones 
- Shaded-in areas to demarcate volume removal

- Dots or small circles to demarcate rotational axis confinement

- Varying line thickness for thicker or thinner line cuts (achieved through focal shift)

Also, given the precision that robot guided laser osteotomy can provide, complex cut shapes not easily realizable with conventional osteotomy tools can be experimented with to potentially enhance healing through increased contact area and/or a decreased need for bone flap fixation hardware (ie. sutures, staples, plates, meshes, dog bones, etc.). Shapes that tesselate and lock together, such as the shape of a jigsaw puzzle piece, could potentially allow for increased mechanical stability during post-op healing.

\section{Nozzle design for assist gas flow}

Delivery of assist gas over the ablation site can augmented for potentially improved affect using different designs for the coaxial delivery nozzle. In this dissertation, the nozzle was kept to a relatively simple conical design and the gas jet speeds were kept subsonic (for obvious reasons). Various nozzle designs (ring, convergent, parallel, etc.) can be experimented with to determine if a change in aero/fluid dynamics during gas-workpiece interaction can improve bone ablation performance.

\section{Understanding safety margins with the use of an absorbing topical chromophore}

The use of a wavelength with small absorption cross section of the substrate (bone) in conjunction with a highly absorbing chromophore has the potential to mitigate unwanted light-to-heat interactions in surrounding tissue, thereby potentially minimizing the HAZ. As well, depositing enough of the chromophore onto the surface of the bone may cause shallower penetration for the photons into the tissue, again reducing the chance of heat generation further into the tissue due to deeper light penetration. 


\subsection{Conclusions}

This research has produced advancements in medical robotics and laser ablation of bone tissue. It is hoped that further developments in this area will result in the replacement and/or augmentation of classical osteotomy techniques and tools with the use of higher-precision mechatronic and photonic methods. It is the author's sincere hope that bone cutting can be made safer, more precise and less traumatic to the patient. 


\section{Appendix A}

\section{Permissions}

\section{A.1 The Optical Society of America (Biomedical Optics Ex- press)}

On Wed, April 24, 2019 at 2:51 PM, pubscopyright wrote:

Dear Jamil Jivraj,

Thank you for contacting The Optical Society.

For the use of material from Jamil Jivraj, Chaoliang Chen, Yize Huang, Joel Ramjist, Yi Lu, Barry Vuong, Xijia Gu, and Victor X. D. Yang, ”Smart laser osteotomy: integrating a pulsed 1064nm fiber laser into the sample arm of a fiber optic 1310nm OCT system for ablation monitoring," Biomed. Opt. Express 9, 6374?6387 (2018):

Because you are the author of the source paper from which you wish to reproduce material, OSA considers your requested use of its copyrighted materials to be permissible within the author rights granted in the Copyright Transfer Agreement submitted by the requester on acceptance for publication of his/her manuscript. It is requested that a complete citation of the original material be included in any publication. This permission assumes that the material was not reproduced from another source when published in the original publication. 
While your publisher should be able to provide additional guidance, OSA prefers the below citation formats: For citations in figure captions:

[Reprinted/Adapted] with permission from ref [x], [Publisher]. (with full citations in reference list) For images without captions:

Journal Vol. \#, first page (year published) An example: Biomed. Opt. Express 9, 6374 (2018)

Please let me know if you have any questions.

Kind Regards,

Rebecca Robinson

Rebecca Robinson

April 24, 2019

Authorized Agent, The Optical Society

The Optical Society (OSA)

2010 Massachusetts Ave., NW Washington, DC 20036 USA www.osa.org 


\section{A.2 SPIE (Optical Engineering)}

Dear Jamil,

Thank you for seeking permission from SPIE to reprint material from our publications. The article you have requested to reprint from is published under Creative Commons (CC BY 4.0)?Gold Open Access. For articles published under CC BY 4.0, users are free to share (copy, distribute, and transmit), to remix (adapt), and to make commercial use of the Article under the following conditions:

Attribution: Users must attribute the contribution in the manner specified by the author or licensor (but not in any way that suggests that they or their use of the Article is endorsed by the Author or licensor). See citation formatting below.

Notice: For any reuse or distribution, users must make clear to others the license terms of this work, preferably using a link to the Creative Commons webpage. The full legal code of the CC BY 4.0 license can be found at https://creativecommons.org/licenses/by/4.0/legalcode.

Citation format (Journals):

Author(s), ?Article Title,? Journal Title and Volume Number (Issue), Article Number (Publication Date). http://dx.doi.org/... Best of luck with your dissertation! Please let me know if I may be of further assistance.

Best,

Katie Sinclair

Editorial Assistant, Publications SPIE 
+13606855436katies@ spie.org

SPIE is the international society for optics and photonics

http://SPIE.org 


\section{A.3 Springer Nature (International Journal of Computer As- sisted Radiology and Surgery)}


This Agreement between Ryerson University -- Jamil Jivraj ("You") and Springer Nature ("Springer Nature") consists of your license details and the terms and conditions provided by Springer Nature and Copyright Clearance Center.

\begin{tabular}{|c|c|}
\hline License Number & 4663140456586 \\
\hline License date & Sep 06, 2019 \\
\hline Licensed Content Publisher & Springer Nature \\
\hline Licensed Content Publication & International Journal of Computer Assisted Radiology and Surgery \\
\hline Licensed Content Title & $\begin{array}{l}\text { Robotic laser osteotomy through penscriptive structured light visual } \\
\text { servoing }\end{array}$ \\
\hline Licensed Content Author & Jamil Jivraj, Ryan Deorajh, Phillips Lai et al \\
\hline Licensed Content Date & Jan 1, 2019 \\
\hline Licensed Content Volume & 14 \\
\hline Licensed Content Issue & 5 \\
\hline Type of Use & Thesis/Dissertation \\
\hline Requestor type & academic/university or research institute \\
\hline Format & electronic \\
\hline Portion & full article/chapter \\
\hline Will you be translating? & no \\
\hline Circulation/distribution & $30-99$ \\
\hline $\begin{array}{l}\text { Author of this Springer } \\
\text { Nature content }\end{array}$ & yes \\
\hline Title & PENSCRIPTIVE DEPTH-CONTROLLED ROBOTIC LASER OSTEOTOMY \\
\hline Institution name & Ryerson University \\
\hline Expected presentation date & Oct 2019 \\
\hline Requestor Location & $\begin{array}{l}\text { Ryerson University } \\
99 \text { Bethany Leigh Drive }\end{array}$ \\
\hline & $\begin{array}{l}\text { Toronto, ON M1V } 2 \mathrm{~N} 6 \\
\text { Canada } \\
\text { Attn: Ryerson University }\end{array}$ \\
\hline Total & $0.00 \mathrm{CAD}$ \\
\hline
\end{tabular}

Terms and Conditions

\section{Springer Nature Customer Service Centre GmbH Terms and Conditions}

This agreement sets out the terms and conditions of the licence (the Licence) between you and Springer Nature Customer Service Centre GmbH (the Licensor). By clicking 'accept' and completing the transaction for the material (Licensed Material), you also confirm your acceptance of these terms and conditions.

\section{Grant of License}

1. 1. The Licensor grants you a personal, non-exclusive, non-transferable, world-wide licence to reproduce the Licensed Material for the purpose specified in your order only. Licences are granted for the specific use requested in the order and for no other use, subject to the conditions below.

1. 2. The Licensor warrants that it has, to the best of its knowledge, the rights to license reuse of the Licensed Material. However, you should ensure that the material you are requesting is original to the Licensor and does not carry the copyright of another entity (as credited in the published version).

1. 3. If the credit line on any part of the material you have requested indicates that it was reprinted or adapted with permission from another source, then you should also seek permission from that source to reuse the material.

\section{Scope of Licence}

2. 1. You may only use the Licensed Content in the manner and to the extent permitted by these Ts\&Cs and any applicable laws.

2. 2. A separate licence may be required for any additional use of the Licensed Material, e.g. where a licence has been purchased for print only use, separate permission must be obtained for electronic re-use. Similarly, a licence is only valid in the language selected and does not apply for editions in other languages unless additional translation rights have been granted separately in the licence. Any content owned by third parties are expressly excluded from the licence.

2. 3. Similarly, rights for additional components such as custom editions and derivatives require additional permission and may be subject to an additional fee. Please apply to 
2. 4. Where permission has been granted free of charge for material in print, permission may also be granted for any electronic version of that work, provided that the material is incidental to your work as a whole and that the electronic version is essentially equivalent to, or substitutes for, the print version.

2. 5. An alternative scope of licence may apply to signatories of the STM Permissions Guidelines, as amended from time to time.

\section{Duration of Licence}

3. 1. A licence for is valid from the date of purchase ('Licence Date') at the end of the relevant period in the below table:

\begin{tabular}{|l||c|}
\hline $\begin{array}{c}\text { Scope of } \\
\text { Licence }\end{array}$ & \multicolumn{1}{c|}{ Duration of Licence } \\
\hline Post on a website & 12 months \\
\hline Presentations & 12 months \\
\hline $\begin{array}{l}\text { Books and } \\
\text { journals }\end{array}$ & $\begin{array}{l}\text { Lifetime of the edition in the language } \\
\text { purchased }\end{array}$ \\
\hline
\end{tabular}

\section{Acknowledgement}

4. 1. The Licensor's permission must be acknowledged next to the Licenced Material in print. In electronic form, this acknowledgement must be visible at the same time as the figures/tables/illustrations or abstract, and must be hyperlinked to the journal/book's homepage. Our required acknowledgement format is in the Appendix below.

\section{Restrictions on use}

5. 1. Use of the Licensed Material may be permitted for incidental promotional use and minor editing privileges e.g. minor adaptations of single figures, changes of format, colour and/or style where the adaptation is credited as set out in Appendix 1 below. Any other changes including but not limited to, cropping, adapting, omitting material that affect the meaning, intention or moral rights of the author are strictly prohibited.

5. 2. You must not use any Licensed Material as part of any design or trademark.

5. 3. Licensed Material may be used in Open Access Publications (OAP) before publication by Springer Nature, but any Licensed Material must be removed from OAP sites prior to final publication.

\section{Ownership of Rights}

6. 1. Licensed Material remains the property of either Licensor or the relevant third party and any rights not explicitly granted herein are expressly reserved.

\section{Warranty}

IN NO EVENT SHALL LICENSOR BE LIABLE TO YOU OR ANY OTHER PARTY OR ANY OTHER PERSON OR FOR ANY SPECIAL, CONSEQUENTIAL, INCIDENTAL OR INDIRECT DAMAGES, HOWEVER CAUSED, ARISING OUT OF OR IN CONNECTION WITH THE DOWNLOADING, VIEWING OR USE OF THE MATERIALS REGARDLESS OF THE FORM OF ACTION, WHETHER FOR BREACH OF CONTRACT, BREACH OF WARRANTY, TORT, NEGLIGENCE, INFRINGEMENT OR OTHERWISE (INCLUDING, WITHOUT LIMITATION, DAMAGES BASED ON LOSS OF PROFITS, DATA, FILES, USE, BUSINESS OPPORTUNITY OR CLAIMS OF THIRD PARTIES), AND

WHETHER OR NOT THE PARTY HAS BEEN ADVISED OF THE POSSIBILITY OF SUCH DAMAGES. THIS LIMITATION SHALL APPLY NOTWITHSTANDING ANY FAILURE OF ESSENTIAL PURPOSE OF ANY LIMITED REMEDY PROVIDED HEREIN.

\section{Limitations}

8. 1. BOOKS ONLY:Where 'reuse in a dissertation/thesis' has been selected the following terms apply: Print rights of the final author's accepted manuscript (for clarity, NOT the published version) for up to 100 copies, electronic rights for use only on a personal website or institutional repository as defined by the Sherpa guideline (www.sherpa.ac.uk/romeo/).

\section{Termination and Cancellation}


9. 1. Licences will expire after the period shown in Clause 3 (above).

9. 2. Licensee reserves the right to terminate the Licence in the event that payment is not received in full or if there has been a breach of this agreement by you.

Appendix 1 - Acknowledgements:

For Journal Content:

Reprinted by permission from [the Licensor]: [Journal Publisher (e.g.

Nature/Springer/Palgrave)] [JOURNAL NAME] [REFERENCE CITATION

(Article name, Author(s) Name), [COPYRIGHT] (year of publication)

For Advance Online Publication papers:

Reprinted by permission from [the Licensor]: [Journal Publisher (e.g.

Nature/Springer/Palgrave)] [JOURNAL NAME] [REFERENCE CITATION

(Article name, Author(s) Name), [COPYRIGHT] (year of publication), advance

online publication, day month year (doi: 10.1038/sj.[JOURNAL ACRONYM].)

For Adaptations/Translations:

Adapted/Translated by permission from [the Licensor]: [Journal Publisher (e.g.

Nature/Springer/Palgrave)] [JOURNAL NAME] [REFERENCE CITATION

(Article name, Author(s) Name), [COPYRIGHT] (year of publication)

Note: For any republication from the British Journal of Cancer, the following credit line style applies:

Reprinted/adapted/translated by permission from [the Licensor]: on behalf of Cancer Research UK: : [Journal Publisher (e.g. Nature/Springer/Palgrave)] [JOURNAL

NAME] [REFERENCE CITATION (Article name, Author(s) Name),

[COPYRIGHT] (year of publication)

For Advance Online Publication papers:

Reprinted by permission from The [the Licensor]: on behalf of Cancer Research UK: [Journal Publisher (e.g. Nature/Springer/Palgrave)] [JOURNAL NAME]

[REFERENCE CITATION (Article name, Author(s) Name), [COPYRIGHT] (year of publication), advance online publication, day month year (doi: 10.1038/sj.

[JOURNAL ACRONYM])

For Book content:

Reprinted/adapted by permission from [the Licensor]: [Book Publisher (e.g.

Palgrave Macmillan, Springer etc) [Book Title] by [Book author(s)]

[COPYRIGHT] (year of publication)

Other Conditions:

Version 1.2

Questions? customercare@copyright.com or +1-855-239-3415 (toll free in the US) or +1-978-646-2777. 


\section{Bibliography}

[1] staff, B., "Medical gallery of balusen medical 2014," WikiJournal of Medicine 1, DOI:10.15347/wjm/2014.010 (ISSN 2002-4436 2014).

[2] Source digital bitmap graphics: BDBRecreated in vector format: Nyq [CC BY-SA 4.0 (https://creativecommons.org/licenses/by-sa/4.0)], from Wikimedia Commons.

[3] Gtirouflet [CC BY-SA 3.0 (https://creativecommons.org/licenses/by sa/3.0)], f. W. C., "Bone normal and degraded micro structure." https://commons.wikimedia.org/ wiki/File:Bone_normal_and_degraded_micro_structure.jpg.

[4] [Public domain], v. W. C., "Illu vertebral column." https://upload.wikimedia. org/wikipedia/commons/f/f8/Illu_vertebral_column.jpg.

[5] user:debivort [CC BY-SA 3.0 (http://creativecommons.org/licenses/by sa/3.0/)], f. W. C., "Cervical vertebra english." commons.wikimedia.org/wiki/File:Cervical_ vertebra_english.png.

[6] Kebes at English Wikipedia [CC BY-SA 3.0 (https://creativecommons.org/licenses/bysa/3.0) or GFDL (http://www.gnu.org/copyleft/fdl.html)], "Absorption spectrum of liquid water." https://upload.wikimedia.org/wikipedia/commons/1/18/ Absorption_spectrum_of_liquid_water.png.

[7] Niemz, M. H., [Laser-tissue interactions: fundamentals and applications], Springer Science \& Business Media (2007). 
[8] Boulnois, J.-L., "Photophysical processes in recent medical laser developments: a review," Lasers in Medical Science 1(1), 47-66 (1986).

[9] Jivraj, J., Huang, Y., Wong, R., Lu, Y., Vuong, B., Ramjist, J., Gu, X., and Yang, V., “Coaxial cavity injected oct and fiber laser ablation system for real-time monitoring of ablative processes," in [Proc. SPIE BiOS], 930505-930505 (2015).

[10] Gresky, J., Batieva, E., Kitova, A., Kalmykov, A., Belinskiy, A., Reinhold, S., and Berezina, N., "New cases of trepanations from the 5th to 3rd millennia bc in southern russia in the context of previous research: Possible evidence for a ritually motivated tradition of cranial surgery?," American journal of physical anthropology 160(4), 665-682 (2016).

[11] Dabis, J., Templeton-Ward, O., Lacey, A. E., Narayan, B., and Trompeter, A., "The history, evolution and basic science of osteotomy techniques," Strategies in Trauma and Limb Reconstruction 12(3), 169-180 (2017).

[12] Kirkup, J., "The history and evolution of surgical instruments. vi. the surgical blade: from finger nail to ultrasound.," Annals of the Royal College of Surgeons of England 77(5), 380 (1995).

[13] Bryant, W. S., “A new motor for bone surgery,” Annals of Surgery 48(2), 303 (1908).

[14] Burgner, J., [Robot assisted laser osteotomy], KIT Scientific Publishing (2010).

[15] Vercellotti, T., "Technological characteristics and clinical indications of piezoelectric bone surgery.," Minerva stomatologica 53(5), 207-214 (2004).

[16] DiMeco, F., Li, K., Mendola, C., Cantu, G., and Solero, C., "Craniotomies without burr holes using an oscillating saw," Acta neurochirurgica 146(9), 995-1001 (2004).

[17] Eddy, H. and Anspach III, W. E., "Perforator," (Mar. 2 1999). US Patent 5,876,405.

[18] Rogers, L. and Lancaster, R., "Dura guard for use with a craniotome and method of use," (Jan. 14 2003). US Patent 6,506,199. 
[19] Engelhardt, M., Uhlenbruch, S., Christmann, A., Miede, C., Eufinger, H., Scholz, M., Harders, A., and Schmieder, K., "Accidential dural tears occurring during supratentorial craniotomy-a prospective analysis of predisposing factors in 100 patients," Zentralblatt für Neurochirurgie 66(02), 70-74 (2005).

[20] Juul, N. and Cold, G. E., "Limitations and complications connected with monitoring of subdural intracranial pressure and insertion of jugular catheter," in [Monitoring of Cerebral and Spinal Haemodynamics During Neurosurgery], 313-318, Springer (2008).

[21] Katzir, A., [Lasers and optical fibers in medicine ], Academic Press Inc., Harcourt Brace \& Company (1993).

[22] Kwoh, Y. S., Hou, J., Jonckheere, E. A., and Hayati, S., "A robot with improved absolute positioning accuracy for ct guided stereotactic brain surgery," Biomedical Engineering, IEEE Transactions on 35(2), 153-160 (1988).

[23] Yiu, G. and He, Z., "Glial inhibition of cns axon regeneration," Nature Reviews Neuroscience 7(8), 617-627 (2006).

[24] Martini, F. et al., [Anatomy and Physiology'2007 Ed], Rex Bookstore, Inc. (1998).

[25] Cancer Research UK and Wikimedia Commons, "Diagram showing craniotomy cruk 063." https://commons.wikimedia.org/wiki/File:Diagram_showing_ a_craniotomy_CRUK_063.svg.

[26] ENCYCLOPÉDIE OU DICTIONNAIRE RAISONNÉ DES SCIENCES, D. A. E. D. M., "Trepanation illustration france 1800s." http://en.wikipedia.org/w/index. php?title=Image:Plate_20_6_20_extract_300px.jpeg.

[27] Giraud, J.-Y., Villemin, S., Darmana, R., Cahuzac, J.-P., Autefage, A., and Morucci, J.-P., "Bone cutting," Clinical Physics and Physiological Measurement 12(1), 1 (1991). 
[28] Caird, J. and Choudhari, K., "'plunging'during burr hole craniostomy: a persistent problem amongst neurosurgeons in britain and ireland," British journal of neurosurgery 17(6), 509512 (2003).

[29] Donovan, D. J., Moquin, R. R., and Ecklund, J. M., "Cranial burr holes and emergency craniotomy: review of indications and technique," Military medicine 171(1), 12-19 (2006).

[30] Ito, M., Sonokawa, T., Mishina, H., and Sato, K., "Penetrating injury of the brain by the burr of a high-speed air drill during craniotomy: case report," Journal of clinical neuroscience 8(3), 261-263 (2001).

[31] Gupta, A. K., Bodhey, N. K., Jayasree, R., Kapilamoorthy, T., Kesavadas, C., Krishnamoorthy, T., Thomas, B., et al., "Percutaneous laser disc decompression: clinical experience at sctimst and long term follow up," Neurology India 54(2), 164 (2006).

[32] Li, Z.-Z., Reinisch, L., and Van de Merwe, W. P., "Bone ablation with er: Yag and co2 laser: study of thermal and acoustic effects," Lasers in surgery and medicine 12(1), 79-85 (1992).

[33] Gertzbein, S., Dedemeter, D., Cruickshank, B., and Kapasouri, A., "The effect of laser osteotomy on bone healing," Lasers in surgery and medicine 1(4), 361-373 (1981).

[34] Wang, X., Zhang, C., and Matsumoto, K., "In vivo study of the healing processes that occur in the jaws of rabbits following perforation by an er, cr: Ysgg laser," Lasers in medical science 20(1), 21-27 (2005).

[35] Stein, E., Sedlacek, T., Fabian, R. L., and Nishioka, N. S., "Acute and chronic effects of bone ablation with a pulsed holmium laser," Lasers in surgery and medicine 10(4), 384-388 (1990).

[36] Yoshino, T., Aoki, A., Oda, S., Takasaki, A. A., Mizutani, K., Sasaki, K. M., Kinoshita, A., Watanabe, H., Ishikawa, I., and Izumi, Y., "Long-term histologic analysis of bone tissue alteration and healing following er: Yag laser irradiation compared to electrosurgery," Journal of periodontology 80(1), 82-92 (2009). 
[37] Keller, U. and Hibst, R., "Lasers in oral surgery," in [International Symposium on Biomedical Optics Europe'94], 146-154, International Society for Optics and Photonics (1994).

[38] Horch, H.-H. and Deppe, H., "New aspects of lasers in oral and craniomaxillofacial surgery," Medical Laser Application 20(1), 7-11 (2005).

[39] Deppe, H. and Horch, H.-H., "Laser applications in oral surgery and implant dentistry," Lasers in medical science 22(4), 217-221 (2007).

[40] Murphy, M. and Torstensson, P., "Thermal relaxation times: an outdated concept in photothermal treatments," Lasers in medical science 29(3), 973-978 (2014).

[41] Nuss, R. C., Fabian, R. L., Sarkar, R., and Puliafito, C. A., "Infrared laser bone ablation,” Lasers in surgery and medicine 8(4), 381-391 (1988).

[42] Gabzdyl, J. T., The effect of laser mode and coaxial gas jet on laser cutting, $\mathrm{PhD}$ thesis, Imperial College London (University of London) (1989).

[43] Steen, W., Watkins, K. G., and Mazumder, J., [Laser material processing], Springer Science \& Business Media (2010).

[44] Fowles, G. R., [Introduction to modern optics], Courier Corporation (2012).

[45] Drexler, W. and Fujimoto, J. G., [Optical coherence tomography: technology and applications ], Springer Science \& Business Media (2008).

[46] Bouma, B., [Handbook of optical coherence tomography], Informa Health Care (2001).

[47] Sakaguchi, T., "Percutaneous puncture with a robot," Hinyokika kiyo. Acta urologica Japonica 31(7), 1265-1268 (1985).

[48] Lechky, O., "World's first surgical robot in b.c.," (November 1985).

[49] Drake, J. M., Joy, M., Goldenberg, A., and Kreindler, D., “Computer-and robot-assisted resection of thalamic astrocytomas in children.," Neurosurgery 29(1), 27-33 (1991). 
[50] Glauser, D., Fankhauser, H., Epitaux, M., Hefti, J.-L., and Jaccottet, A., "Neurosurgical robot minerva: first results and current developments," Journal of Image Guided Surgery 1(5), 266-272 (1995).

[51] Sautot, P., Cinquin, P., Lavallee, S., and Troccaz, J., "Computer assisted spine surgery: A first step toward clinical, application in orthopaedics," in [Engineering in Medicine and Biology Society, 1992 14th Annual International Conference of the IEEE], 3, 1071-1072, IEEE (1992).

[52] Tian, Z., Lu, W., Wang, T., Ma, B., Zhao, Q., and Zhang, G., "Application of a robotic telemanipulation system in stereotactic surgery," Stereotactic and functional neurosurgery 86(1), 54-61 (2008).

[53] Gao, X., "The anatomy of teleneurosurgery in china," International journal of telemedicine and applications 2011, 9 (2011).

[54] Wu, Z., Zhao, Q., Tian, Z., Zhang, J., Xiao, X., Lin, H., Wang, H., and Wang, F., "Efficacy and safety of a new robot-assisted stereotactic system for radiofrequency thermocoagulation in patients with temporal lobe epilepsy," Experimental and therapeutic medicine 7(6), 17281732 (2014).

[55] Ortmaier, T., "Kinemedic: Robot assisted placement of pedicle screws," Proc. 2nd Summer European University, Montpellier, France (2005).

[56] Mitsuishi, M., Morita, A., Sugita, N., Sora, S., Mochizuki, R., Tanimoto, K., Baek, Y. M., Takahashi, H., and Harada, K., "Master-slave robotic platform and its feasibility study for micro-neurosurgery," The International Journal of Medical Robotics and Computer Assisted Surgery 9(2), 180-189 (2013).

[57] Takasuna, H., Goto, T., Kakizawa, Y., Miyahara, T., Koyama, J., Tanaka, Y., Kawai, T., and Hongo, K., "Use of a micromanipulator system (neurobot) in endoscopic neurosurgery," Journal of Clinical Neuroscience 19(11), 1553-1557 (2012). 
[58] Heinig, M., Govela, M. F., Gasca, F., Dold, C., Hofmann, U. G., Tronnier, V., Schlaefer, A., and Schweikard, A., "Mars-motor assisted robotic stereotaxy system," in [Neural Engineering (NER), 2011 5th International IEEE/EMBS Conference on], 334-337, IEEE (2011).

[59] Heinig, M., Design and evaluation of the motor assisted robotic stereotaxy system MARS, PhD thesis, Zentrale Hochschulbibliothek Lübeck (2012).

[60] Heinig, M., Hofmann, U. G., and Schlaefer, A., "Calibration of the motor-assisted robotic stereotaxy system: Mars," International journal of computer assisted radiology and surgery 7(6), 911-920 (2012).

[61] Arata, J., Kenmotsu, H., Takagi, M., Hori, T., Miyagi, T., Fujimoto, H., Kajita, Y., Hayashi, Y., Chinzei, K., and Hashizume, M., "Surgical bedside master console for neurosurgical robotic system," International journal of computer assisted radiology and surgery 8(1), 7586 (2013).

[62] Grau, C., Ginhoux, R., Riera, A., Nguyen, T. L., Chauvat, H., Berg, M., Amengual, J. L., Pascual-Leone, A., and Ruffini, G., "Conscious brain-to-brain communication in humans using non-invasive technologies," PLoS One 9(8), e105225 (2014).

[63] Zhang, Y., [Optical Coherence Tomography Guided Laser-Cochleostomy], KIT Scientific Publishing (2015).

[64] Burgner, J., Toma, M., Vieira, V., Eggers, G., Raczkowsky, J., Mühling, J., Marmulla, R., and Wörn, H., "System for robot assisted orthognathic surgery,"

[65] Burgner, J., Raczkowsky, J., and Wörn, H., "Establishment of an experimental setup for robot assisted laser osteotomy with corresponding simulation environment for optimization of all relevant parameters," Proceedings of the curac 8, 147-148 (2008).

[66] Burgner, J., Zhang, Y., Raczkowsky, J., Woern, H., Eggers, G., and Muehling, J., “Meth- 
ods for end-effector coupling in robot assisted interventions," in [Robotics and Automation, 2008. ICRA 2008. IEEE International Conference on ], 3395-3400, IEEE (2008).

[67] Burgner, J., Kahrs, L. A., Raczkowsky, J., and Wörn, H., "Including parameterization of the discrete ablation process into a planning and simulation environment for robot-assisted laser osteotomy," in [Medicine meets virtual reality], 17, 43-48 (2009).

[68] Burgner, J., Knapp, F., Kahrs, L., Raczkowsky, J., Wörn, H., Schipper, J., and Klenzner, T., "Setup and experimental trial for robot-assisted laser cochleostomy," International Journal of Computer Assisted Radiology and Surgery 4(Suppl 1), 118-119 (2009).

[69] Burgner, J., Raczkowsky, J., and Wörn, H., "End-effector calibration and registration procedure for robot assisted laser material processing: Tailored to the particular needs of short pulsed co 2 laser bone ablation," in [Robotics and Automation, 2009. ICRA'09. IEEE International Conference on ], 3091-3096, IEEE (2009).

[70] Burgner, J., Mueller, M., Raczkowsky, J., and Woern, H., "Robot assisted laser bone processing: Marking and cutting experiments," in [Advanced Robotics, 2009. ICAR 2009. International Conference on ], 1-6, IEEE (2009).

[71] Zhang, Y., Burgner, J., Raczkowsky, J., and Wörn, H., “Optimization of line cut strategy for bone tissue ablation using short-pulsed co2 laser.,"

[72] Kahrs, L. A., Burgner, J., Klenzner, T., Raczkowsky, J., Schipper, J., and Wörn, H., "Planning and simulation of microsurgical laser bone ablation," International journal of computer assisted radiology and surgery 5(2), 155-162 (2010).

[73] Burgner, J., "Robotic guidance for microsurgical laser bone processing in ent surgery," (2010).

[74] Augello, M., Baetscher, C., Segesser, M., Zeilhofer, H.-F., Cattin, P., and Juergens, P., "Performing partial mandibular resection, fibula free flap reconstruction and midfacial osteotomies with a cold ablation and robot-guided er: Yag laser osteotome (carlo®)-a study 
on applicability and effectiveness in human cadavers," Journal of Cranio-Maxillofacial Surgery 46(10), 1850-1855 (2018).

[75] Deibel, W., Schneider, A., Augello, M., Bruno, A. E., Juergens, P., and Cattin, P., “A compact, efficient, and lightweight laser head for carlo®: integration, performance, and benefits," in [Novel Optical Systems Design and Optimization XVIII], 9579, 957905, International Society for Optics and Photonics (2015).

[76] Shoham, M., Burman, M., Zehavi, E., Joskowicz, L., Batkilin, E., and Kunicher, Y., "Bonemounted miniature robot for surgical procedures: Concept and clinical applications," IEEE Transactions on Robotics and Automation 19(5), 893-901 (2003).

[77] (June 2011).

[78] Liu, Y., "Potential risk of intelligent technologies in clinical orthopedics," in [Intelligent Orthopaedics ], 281-288, Springer (2018).

[79] Ravi, B., Zahrai, A., and Rampersaud, R., "Clinical accuracy of computer-assisted twodimensional fluoroscopy for the percutaneous placement of lumbosacral pedicle screws," Spine 36(1), 84-91 (2011).

[80] Khan, A., Meyers, J. E., Siasios, I., and Pollina, J., "Next-generation robotic spine surgery: First report on feasibility, safety, and learning curve," Operative Neurosurgery (2018).

[81] Medical, G., "Globus medical announces fda 510(k) clearance for excelsius gps ${ }^{\mathrm{TM}}$," (August 2017).

[82] Jiang, B., Ahmed, A. K., Zygourakis, C. C., Kalb, S., Zhu, A. M., Godzik, J., Molina, C. A., Blitz, A. M., Bydon, A., Crawford, N., et al., "Pedicle screw accuracy assessment in excelsiusgps® robotic spine surgery: evaluation of deviation from pre-planned trajectory," Chinese Neurosurgical Journal 4(1), 23 (2018).

[83] Yasin, H., Hoff, H.-J., Blümcke, I., and Simon, M., "Experience with 102 frameless stereotactic biopsies using the neuromate robotic device," World neurosurgery (2018). 
[84] Abhinav, K., Prakash, S., and Sandeman, D. R., "Use of robot-guided stereotactic placement of intracerebral electrodes for investigation of focal epilepsy: initial experience in the uk," British journal of neurosurgery 27(5), 704-705 (2013).

[85] Châtillon, C.-E., Mok, K., Hall, J., and Olivier, A., "Comparative study of manual versus robot-assisted frameless stereotaxy for intracranial electrode implantation,” (12 2011).

[86] Surgical, I., “About intuitive.”

[87] Surgery, D. V., “Abdominal conditions.”

[88] Health, A., "Robotic assisted surgery - mercy hospital - unity campus - surgery types."

[89] Jivraj, J., Chen, C., Huang, Y., Ramjist, J., Lu, Y., Vuong, B., Gu, X., and Yang, V. X., "Smart laser osteotomy: integrating a pulsed 1064nm fiber laser into the sample arm of a fiber optic 1310nm oct system for ablation monitoring," Biomedical Optics Express 9(12), 6374-6387 (2018).

[90] Kahrs, L. A., Burgner, J., Klenzner, T., Raczkowsky, J., Schipper, J., and Wörn, H., "Planning and simulation of microsurgical laser bone ablation," International journal of computer assisted radiology and surgery 5(2), 155-162 (2010).

[91] Stopp, S., Svejdar, D., Von Kienlin, E., Deppe, H., and Lueth, T. C., “A new approach for creating defined geometries by navigated laser ablation based on volumetric 3-d data," Biomedical Engineering, IEEE Transactions on 55(7), 1872-1880 (2008).

[92] Kahrs, L. A., Raczkowsky, J., Werner, M., Knapp, F. B., Mehrwald, M., Hering, P., Schipper, J., Klenzner, T., and Wörn, H., "Visual servoing of a laser ablation based cochleostomy," in [Medical imaging], 69182C-69182C, International Society for Optics and Photonics (2008).

[93] Ohmi, M., Tanizawa, M., Fukunaga, A., and Haruna, M., "In-situ observation of tissue laser ablation using optical coherence tomography," Optical and quantum electronics 37(13-15), 1175-1183 (2005). 
[94] Ohmi, M., Ohnishi, M., Takada, D., and Haruna, M., "Real-time oct imaging of laser ablation of biological tissue," in [BiOS], 756210-756210, International Society for Optics and Photonics (2010).

[95] Oh, W., Yun, S., Vakoc, B., Tearney, G., and Bouma, B., "Ultrahigh-speed optical frequency domain imaging and application to laser ablation monitoring," Applied Physics Letters 88(10), 103902-103902 (2006).

[96] Ohnishi, M., Takada, D., Ohmi, M., and Haruna, M., "Dynamic analysis of laser ablation of biological tissue using a real-time oct," in [SPIE BiOS: Biomedical Optics], 717513717513, International Society for Optics and Photonics (2009).

[97] Torkian, B. A., Guo, S., Jahng, A. W., Liaw, L.-H. L., Chen, Z., and Wong, B. J., "Noninvasive measurement of ablation crater size and thermal injury after co2 laser in the vocal cord with optical coherence tomography," Otolaryngology-Head and Neck Surgery 134(1), 86-91 (2006).

[98] Webster, P. J., Muller, M. S., and Fraser, J. M., "High speed in situ depth profiling of ultrafast micromachining," Optics express 15(23), 14967-14972 (2007).

[99] Webster, P. J., Leung, B. Y., Joe, X., Anderson, M. D., Hoult, T. P., and Fraser, J. M., "Coaxial real-time metrology and gas assisted laser micromachining: process development, stochastic behavior, and feedback control," in [MOEMS-MEMS], 759003-759003, International Society for Optics and Photonics (2010).

[100] Leung, B. Y., Webster, P. J., Fraser, J. M., and Yang, V. X., "Real-time guidance of thermal and ultrashort pulsed laser ablation in hard tissue using inline coherent imaging," Lasers in surgery and medicine 44(3), 249-256 (2012).

[101] Beaudette, K., Baac, H. W., Madore, W.-J., Villiger, M., Godbout, N., Bouma, B. E., and Boudoux., C., "Laser tissue coagulation and concurrent optical coherence tomography through a double-clad fiber coupler.," Biomedical Optics Express 6(4), 1293-1303 (2015). 
[102] Beaudette, K., Lo, W., Villiger, M., Shishkov, M., Godbout, N., Bouma, B. E., and Boudoux., C., "Towards in vivo laser coagulation and concurrent optical coherence tomography through double-clad fiber devices," in [Multimodal Biomedical Imaging XI], 9701, 97010B, International Society for Optics and Photonics (March 2016).

[103] Jivraj, J., Zhou, J., Gu, X. J., and Yang, V. X., "Coaxial cavity injected oct and fiber laser ablation system for real-time monitoring of ablative processes part 2: toward all fiber design and dispersion control (conference presentation)," in [Clinical and Translational Neurophotonics ], 10050, 100500C, International Society for Optics and Photonics (2017).

[104] Lu, Y. and Gu, X., "All-fiber passively q-switched fiber laser with a sm-doped fiber saturable absorber," Optics express 21(2), 1997-2002 (2013).

[105] Incorporated, C., "Corning smf-28e+ optical fiber product information," specification sheet, Corning Incorporated, New York, USA (2014).

[106] Wojtkowski, M., Srinivasan, V., Ko, T., Fujimoto, J., Kowalczyk, A., and Duker, J., "Ultrahigh-resolution, high-speed, fourier domain optical coherence tomography and methods for dispersion compensation," Optics express 12(11), 2404-2422 (2004).

[107] Lee, K. K., Mariampillai, A., Joe, X., Cadotte, D. W., Wilson, B. C., Standish, B. A., and Yang, V. X., "Real-time speckle variance swept-source optical coherence tomography using a graphics processing unit," Biomedical optics express 3(7), 1557-1564 (2012).

[108] Barrows, D., Vuong, B., Lee, K., Jivraj, J., and Yang, V. X., "Graphics processor unit acceleration enables realtime endovascular doppler optical coherence tomography imaging," in [Optical Coherence Tomography and Coherence Domain Optical Methods in Biomedicine XXI], 10053, 100532G, International Society for Optics and Photonics (2017).

[109] Jivraj, J., Deorajh, R., Lai, P., Chen, C., Nguyen, N., Ramjist, J., and Yang, V. X., "Robotic laser osteotomy through penscriptive structured light visual servoing," International journal of computer assisted radiology and surgery , 1-10 (2019). 
[110] AG, A. O. T., "Advanced osteotomy tools."

[111] Vadera, S., Chan, A., Low, T., Gill, A., Morenkova, A., Phiellip, N. M., Hermanowicz, N., and Hsu, F. P., "Frameless stereotactic robot-assisted subthalamic nucleus deep brain stimulation: case report.," World Neurosurgery 97, 762-e11 (Janurary 2017).

[112] Giger, A., Jud, C., and Cattin, P., "Respiratory motion compensation for the robotguided laser osteotome," International Journal of Computer Assisted Radiology and Surgery 12(10), 1751-1762 (2017).

[113] Burgner, J., "Robotic assisted laser osteotmy," KIT Scientific Publishing (2010).

[114] Baek, K.-W., Deibel, W., Martinov, D., Griessen, M., Bruno, A., Zeilhofer, H.-F., Cattin, P., and Juergens, P., "Clinical applicability of robot-guided contact-free laser osteotomy in cranio-maxillo-facial surgery: in-vitro simulation and in-vivo surgery in minipig mandibles," Journal of Oral and Maxillofacial Surger 53(10), 976-981 (2015).

[115] Deibel, W., Schneider, A., Augello, M., Bruno, A. E., Juergens, P., and Cattin, P., [A compact, efficient and light weight laser head for CARLO®: integration, performance and benefits ], vol. 9579, International Society for Optics and Photonics (September 2015).

[116] Mönnich, H., Stein, D., Raczkowsky, J., and Wörn, H., "Results of co2 robotic laser oseotomy in surgery with motion compensation," Photonic Therapeutics and Diagnostics VI 7548 (2010).

[117] Xu, D., andLinkun Wang, Z. J., and Tan, M., "Features extraction for structured light image of welding seam with arc and splash disturbance," in [8th International Conference on Controt, Automation. Robotics and Vision ], (December 2004).

[118] Xu, D., Wang, L., and Tan, M., "Image processing and visual control method for arc welding robot," in [International Conference on Robotics and Biomimetics], 727-732 (2004). 
[119] Xu, D., Tan, M., and Li, Y., [Industrial Robotics Theory Modelling Control], ch. Visual Control System for Robotic Welding, Pro Literatur Verlag, Germany / ARS, Austria, Germany (December 2006).

[120] Xu, D., Tan, M., Zhao, X., and Tu, Z., "Seam tracking and visual control for robotic arc welding based on structured light stereovision," International Journal of Automation and Computing 1, 63-75 (2004).

[121] Yu, Z., He, Y., Xu, Y., and Chen, H., "Vision-based deviation extraction for threedimensional control in robotic welding with steel sheet," Internationa Journal of Advanced Manufacturing Technology 95(9-12), 4449-4458 (2018).

[122] Zou, Z. Y. . G. F. . S. C. . J. J., "Passive vision based seam tracking system for pulsemag welding," Internationa Journal of Advanced Manufacturing Technology 67, 1987-1996 (2012).

[123] Xu, Y., Fang, G., Chen, S., Zou, J. J., and Ye, Z., "Real-time image processing for visionbased weld seam tracking in robotic gmaw," Internationa Journal of Advanced Manufacturing Technology 73, 1413-1425 (May 2014).

[124] Guha, D., Gupta, S., Fehlings, M. G., and Yang, V., “Optical topographic imaging for spinal intraoperative three-dimensional navigation in minimally invasive approaches: Initial preclinical experience," The Spine Journal 17(10), S254 (2017).

[125] Sciavicco, L. and Siciliano, B., [Modelling and Control of Robot Manipulators], Springer, 2 ed. (2000).

[126] Boomgaard, R. V. D. and Balen., R. V., "Methods for fast morphological image transforms using bitmapped images," Computer Vision, Graphics, and Image Processing: Graphical Models and Image Processing 54, 254-258 (May 1992).

[127] Sobel, I. and Feldman, G., "A 3x3 isotropic gradient operator for image processing," a talk at the Stanford Artificial Project in , 271-272 (1968). 
[128] Zill, D. and Cullen, M., [Advanced Engineering Mathematics], Jones \& Bartlett Learning, 3 ed. (February 2006).

[129] Jolliffe, I., [Principal Component Analysis, Series: Springer Series in Statistics], Springer, NY, 2 ed. (2002).

[130] Wiles, A., Thompson, D., and Frantz, D., “Accuracy assessment and interpretation for optical tracking systems," in [Proceedings of SPIE Medical Imaging 2004: Visualization, Image-Guided Procedures and Display], 5367, 421-432, SPIE (February 2004).

[131] Elfring, R., de la Fuente, M., and Radermarcher, K., "Assessment of optical localizer accuracy for computer aided surgery systems," Computer Aided Surgery 15(1-3), 1-12 (2010).

[132] International Society for Optics and Photonics, [Preliminary Testing of a Compact, BoneAttached Robot for Otologic Surgery], 9036 (2014).

[133] Dillion, N. P., Balachandran, R., Fitzpatrick, J. M., Siebold, M. A., Labadie, R. F., Wanna, G. B., Withrow, T. J., and III, R. J. W., “A compact, bone-attached robot for mastoidectomy,” Journal of Medical Devices 9(3), 031003 (2015).

[134] Gerber, N., Bell, B., Gavaghan, K., Weisstanner, C., Caversaccio, M., and Weber, S., “Surgical planning tool for robotically assisted hearing aid implantation," International Journal of Computer Assisted Radiology and Surgery 9(1), 11-20 (2014).

[135] Zagzoog, N. and Yang, V. X., "State of robotic mastoidectomy: Literature review," World Neurosurgery (June 2018).

[136] Jivraj, J., Chen, C., Barrows, D., Gu, X., and Yang, V. X., “Optimization of laser osteotomy at 1064 nm using a graphite topical absorber and a nitrogen assist gas jet," Biomedical Optics Express 10(7), 3114-3123 (2019).

[137] Sumian, C. C., Pitre, F. B., Gauthier, B. E., Bouclier, M., and Mordon, S. R., "Laser skin resurfacing using a frequency doubled nd: Yag laser after topical application of an exoge- 
nous chromophore," Lasers in Surgery and Medicine: The Official Journal of the American Society for Laser Medicine and Surgery 25(1), 43-50 (1999).

[138] Diven, D. G., Pohl, J., and Motamedi, M., "Dye-enhanced diode laser photothermal ablation of skin," Journal of the American Academy of Dermatology 35(2), 211-215 (1996).

[139] Mordon S., Desmettre T., D. J. M. . M. V., "Selective laser photocoagulation of blood vessels in a hamster skin flap model using a specific icg formulation," Lasers in surgery and medicine 21(4), 365-373 (1997).

[140] Roh, Mi Ryung, H. J. C. and Chung, K. Y., "Effects of various parameters of the 1064 nm nd: Yag laser for the treatment of enlarged facial pores," Journal of Dermatological Treatment 20(4), 223-228 (2009).

[141] Kang, H. W., lee, H., Chen, S., and Welch, A. J., "Enhancement of bovine bone ablation assisted by a transparent liquid layer on a target surface," IEEE Journal of Quantum Electronics 42, 633-642 (July 2006).

[142] Hoy, C. L., Ferhanoğlu, O., Yildirim, M., Kim, K. H., Karajanagi, S. S., Chan, K. M. C., Kobler, J. B., Zeitels, S. M., and Ben-Yakar, A., "Clinical ultrafast laser surgery: recent advances and future directions," IEEE Journal of Selected Topics in Quantum Electronics 20(2), 242-255 (2014).

[143] Webster, P. J., Wright, L. G., Mortimer, K. D., Leung, B. Y., Joe, X., and Fraser, J. M., "Automatic real-time guidance of laser machining with inline coherent imaging," Journal of Laser Applications 23(2), 022001 (2011).

[144] Eriksson, A. and Albrektsson, T., "Temperature threshold levels for heat-induced bone tissue injury: a vital-microscopic study in the rabbit," Journal of prosthetic dentistry 50(1), 101107 (1983).

[145] Nelson, J. S., Orenstein, A., Liaw, L.-H. L., and Berns, M. W., "Mid-infrared erbium: Yag 
laser ablation of bone: The effect of laser osteotomy on bone healing," Lasers in surgery and medicine 9(4), 362-374 (1989).

[146] Yoshida, K., Uoshima, K., Oda, K., and Maeda, T., "Influence of heat stress to matrix on bone formation," Clinical Oral Implants Research, no-no (2009).

[147] Rajitha Gunaratne, G., Khan, R., Fick, D., Robertson, B., Dahotre, N., and Ironside, C., "A review of the physiological and histological effects of laser osteotomy," Journal of medical engineering \& technology 41(1), 1-12 (2017).

[148] Sotsuka, Y., Nishimoto, S., Tsumano, T., Kawai, K., Ishise, H., Kakibuchi, M., Shimokita, R., Yamauchi, T., and Okihara, S.-i., "The dawn of computer-assisted robotic osteotomy with ytterbium-doped fiber laser," Lasers in medical science 29(3), 1125-1129 (2014).

[149] Webb, H., Lubner, M. G., and Hinshaw, J. L., "Thermal ablation," in [Seminars in roentgenology], 46(2), 133-141, Elsevier (2011).

[150] Hwang, J., Kim, H., Truong, G. V., Xuan, J., Hasenberg, T., and Kang, H. W., "Dualwavelength-assisted thermal hemostasis for treatment of benign prostate hyperplasia," Journal of biophotonics 11(4), e201700192 (2018).

[151] Lee, S. J. and Ha, H. J., "In vivo measurement of blood flow in a micro-scale stenosis model generated by laser photothermal blood coagulation," IET systems biology 7(2), 5055 (2013).

[152] Giglio, N. C., Hutchens, T. C., Perkins, W. C., Latimer, C., Ward, A. K., Nau, W. H., and Fried, N. M., "Rapid sealing and cutting of porcine blood vessels, ex vivo, using a highpower, 1470-nm diode laser," Journal of biomedical optics 19(3), 038002 (2014).

[153] Jivraj, J., Deorajh, R., Lai, P., Chen, C., Nguyen, N., Ramjist, J., and Yang, V. X., "Robotic laser osteotomy through penscriptive structured light visual servoing," International journal of computer assisted radiology and surgery , 1-10 (2019). 
[154] Wong, R., Jivraj, J., Vuong, B., Ramjist, J., Dinn, N. A., Sun, C., Huang, Y., Smith, J. A., and Yang, V. X., "Development of an integrated optical coherence tomography-gas nozzle system for surgical laser ablation applications: preliminary findings of in situ spinal cord deformation due to gas flow effects," Biomedical Optics Express 6, 43-53 (January 2015).

[155] Aljekhedab, F., Zhang, W., Favero, J., Haugen, H. K., Wohl, G. R., and Fang, Q., “Bovine cortical bone ablation by femtosecond laser (conference presentation)," in [Lasers in Dentistry XXIV], 10473, 104730F, International Society for Optics and Photonics (2018).

[156] Nelson, J. S., Orenstein, A., Liaw, L.-H. L., and Berns, M. W., "Mid-infrared erbium: Yag laser ablation of bone: The effect of laser osteotomy on bone healing," Lasers in surgery and medicine 9(4), 362-374 (1989).

[157] Buchelt, M., Kutschera, H.-P., Katterschafka, T., Kiss, H., Lang, S., Beer, R., and Losert, U., "Erb: Yag and hol: Yag laser osteotomy: the effect of laser ablation on bone healing," Lasers in surgery and medicine 15(4), 373-381 (1994).

[158] Stein, E., Sedlacek, T., Fabian, R. L., and Nishioka, N. S., "Acute and chronic effects of bone ablation with a pulsed holmium laser," Lasers in surgery and medicine 10(4), 384-388 (1990).

[159] Jivraj, J., Chen, C., Barrows, D., and Yang, V. X., “Optical coherence tomography for dynamic axial correction of an optical end-effector for robot-guided surgical laser ablation," Optical Engineering 58(5), 054106 (2019).

[160] Steen, W. and Mazumder, J., [Laser Material Processing], Springer Science and Business Media (2010).

[161] Cao, B. X., Bae, M., Sohn, H., Choi, J., Kim, Y., o Kim, J., and Noh, J., “Design and performance of a focus-detection system for use in laser micromachining," Micromachines 7(1), 2 (2016). 
[162] Grosjean, T. and Courjon, D., "Smallest focal spots," Optics Communications 272(2), 314319 (2007).

[163] Hand, D., Fox, M., Haran, F., Peters, C., Morgan, S., McLean, M., Steen, W., and Jones, J., "Optical focus control system for laser welding and direct casting," Optics and lasers in Engineering 34, 415-427 (October 2000).

[164] Cao, B. X., Hoang, P. L., Ahn, S., o Kim, J., Sohn, H., and Noh, J., "Real-time detection of focal position of workpiece surface during laser processing using diffractive beam samplers," Optics and Lasers in Engineering 86, 92-97 (2016).

[165] Cao, B. X., Hoang, P. L., Ahn, S., o Kim, J., Kang, H., and Noh, J., "In-situ real-time focus detection during laser processing using double-hole masks and advanced image sensor software," Sensors 17, 1540 (July 2017).

[166] Cao, B. X., Hoang, P. L., Ahn, S., o Kim, J., Kang, H., and Noh, J., "Real-time laser focusing system for high-precision micromachining using diffractive beam sampler and advanced image sensor," Optics and Lasers in Engineering 107, 13-20 (2018).

[167] Yu, H., Shen, J.-H., Joos, K. M., and Simaan, N., "Design, calibration and preliminary testing of a robotic telemanipulator for oct guided retinal surgery," in [2013 IEEE International Conference on Robotics and Automation (ICRA)], IEEE (May 2013).

[168] Joos, K. M. and Shen, J.-H., "Preliminary design and evaluation of a b-scan oct-guided needle," Photonics 1(3), 260-266 (2014).

[169] Yu, H., Shen, J.-H., Shah, R. J., Simaan, N., and Joos, K. M., "Evaluation of microsurgical tasks with oct-guided and/or robot-assisted ophthalmic forceps," Biomedical Optics Express 6, 457-472 (February 2015).

[170] Yu, H., Shen, J.-H., Joos, K. M., and Simaan, N., "Calibration and integration of b-mode optical coherence tomography for assistive control in robotic microsurgery," IEEE/ASME TRANSACTIONS ON MECHATRONICS 21, 2613-2623 (December 2016). 
[171] Cheon, G. W., Huang, Y., Cha, J., Gehlbach, P. L., and Kang, J. U., "Accurate real-time depth control for cp-ssoct distal sensor based handheld microsurgery tools," Biomedical Optics Express 6(5), 1942-1953 (2015).

[172] Boppart, S. A., Herrmann, J., Pitris, C., Stamper, D. L., Brezinski, M. E., and Fujimoto, J. G., "High-resolution optical coherence tomography-guided laser ablation of surgical tissue,” Journal of Surgical Research 82(2), 275-284 (1999).

[173] Ji, Y., Inline Coherent Imaging Applied to Laser Micromachining, Master's thesis (2014).

[174] KUKA-Roboter-GmbH, “Kuka senstive robotics lbr iiwa.” PDF Brochure (2017).

[175] Davidson, S. R. and James, D. F., "Drilling in bone: modeling heat generation and temperature distribution,” Journal of Biomechanical Engineering 125(3), 305-314 (2003).

[176] Fercher, A. and Roth, E., "Ophthalmic laser interferometry," in [Optical instrumentation for biomedical laser applications ], 658, 48-52, International Society for Optics and Photonics (1986).

[177] Fercher, A., Mengedoht, K., and Werner, W., "Eye-length measurement by interferometry with partially coherent light," Optics Letters 13, 186-188 (March 1988).

[178] Fercher, A., "Ophthalmic interferometry," in [Optics in Medicine, Biology and Environmental Research First International Conference on Optics Within Life Sciences (OWLS I)], 12-16 (1990).

[179] Chiba, S. and Tanno, N., "Backscattering optical heterodyne tomography," in [prepared for the 14th Laser Sensing Symposium], (1991).

[180] Huang, D., Swanson, E. A., Lin, C. P., Schuman, J. S., Stinson, W. G., Chang, W., Hee, M. R., Flotte, T., Gregory, K., Puliafito, C. A., and Fujimoto, J. G., "Optical coherence tomography," Science 254, 1178-1181 (November 1991). 
[181] Gambichler, T., Jaedicke, V., and Terras, S., "Optical coherence tomography in dermatology," Archives in dermatological research 303, 457-473 (September 2001).

[182] Sattler, E. C., Kästle, R., and Welzel, J., “Optical coherence tomography in dermatology,” Journal of biomedical optics 18(6), 061224 (2013).

[183] Holmes, J. and Welzel, J., “Oct in dermatology,” Optical Coherence Tomography: Technology and Applications , 2189-2207 (2015).

[184] Jang, I., Bouma, B., Kang, D., Park, S., Park, S., Seung, K., Choi, K., Shishkov, M., Schlendorf, K., Pomeranstev, E., and Houser, S., "Visualization of coronary atherosclerotic plaques in patients using optical coherence tomography: comparison with intravascular ultrasound," Journal of American College of Cardiology 39, 604-609 (February 2002).

[185] Bezerra, H. G., Costa, M. A., Guagliumi, G., Rollins, A. M., and Simon, D. I., "ntracoronary optical coherence tomography: a comprehensive review: clinical and research applications," JACC: Cardiovascular Interventions 2, 1035-1046 (November 2009).

[186] Jones, D., Rathod, K., Koganti, S., Hamshere, S., Ashtroulakis, Z., Lim, P., Sirker, A., O’Mahony, C., Jain, A., Knight, C., and Dalby, M., “Angiography alone versus angiography plus optical coherence tomography to guide percutaneous coronary intervention: outcomes from the pan-london pci cohort," JACC: Cardiovascular Interventions 11, 1313-1321 (July 2018).

[187] Lee, H.-C., Liang, K., Ahsen, O. O., Wang, Z., Figueiredo, M., Potsaid, B., Jayaraman, V., Huang, Q., Mashimo, H., and Fujimoto, J. G., "Endoscopic optical coherence tomography and angiography for gastroenterology applications," in [Biomedical Imaging and Sensing Conference ], 10711, 107110A, International Society for Optics and Photonics (2018).

[188] Dong, J., Verma, Y., Farrokhi, H., Otuya, D., Giddings, S., Vuong, B., Bhat, N., Shakil, O., Grant, C. N., Rosenberg, M., et al., "Tu1967-unsedated transnasal optical coherence tomography (oct) endomicroscopy of the small intestine," Gastroenterology 154(6), S-1068 (2018). 
[189] Kassinopoulos, M., Dong, J., Tearney, G. J., and Pitris, C., "Automated detection of esophageal dysplasia in in vivo optical coherence tomography images of the human esophagus," in [Optical Coherence Tomography and Coherence Domain Optical Methods in Biomedicine XXII], 10483, 104830R, International Society for Optics and Photonics (2018). 